\title{
Signaling games : theory and applications
}

Citation for published version (APA):

Lee, J. (2013). Signaling games : theory and applications. [Doctoral Thesis, Maastricht University]. Universitaire Pers Maastricht. https://doi.org/10.26481/dis.20130617jl

Document status and date:

Published: 01/01/2013

DOI:

10.26481/dis.20130617jl

Document Version:

Publisher's PDF, also known as Version of record

\section{Please check the document version of this publication:}

- A submitted manuscript is the version of the article upon submission and before peer-review. There can be important differences between the submitted version and the official published version of record.

People interested in the research are advised to contact the author for the final version of the publication, or visit the DOI to the publisher's website.

- The final author version and the galley proof are versions of the publication after peer review.

- The final published version features the final layout of the paper including the volume, issue and page numbers.

Link to publication

\footnotetext{
General rights rights.

- You may freely distribute the URL identifying the publication in the public portal. please follow below link for the End User Agreement:

www.umlib.nl/taverne-license

Take down policy

If you believe that this document breaches copyright please contact us at:

repository@maastrichtuniversity.nl

providing details and we will investigate your claim.
}

Copyright and moral rights for the publications made accessible in the public portal are retained by the authors and/or other copyright owners and it is a condition of accessing publications that users recognise and abide by the legal requirements associated with these

- Users may download and print one copy of any publication from the public portal for the purpose of private study or research.

- You may not further distribute the material or use it for any profit-making activity or commercial gain

If the publication is distributed under the terms of Article $25 \mathrm{fa}$ of the Dutch Copyright Act, indicated by the "Taverne" license above, 
Signaling Games: Theory and Applications 
Signaling Games: Theory and Applications

(C) Copyright Jiwoong LEE, Maastricht 2013

All rights reserved. No part of this publication may be reproduced, stored in a retrieval system, or transmitted, in any form, or by any means, electronic, mechanical, photocopying, recording or otherwise, without the prior permission in writing from the author.

This book was typeset by the author using LATEX.

Cover photo by Bien-U BAE. Cover design by Jiwoong LEE.

Published by Universitaire Pers Maastricht

ISBN 9789461592378

Printed in the Netherlands by Datawyse 


\title{
Signaling Games: Theory and Applications
}

\author{
Proefschrift
}

ter verkrijging van de graad van doctor aan de Universiteit Maastricht, op gezag van Rector Magnificus, Prof. dr. L.L.G. Soete, volgens het besluit van het College van Decanen, in het openbaar te verdedigen op maandag 17 juni 2013, om 12:00 uur

door

\section{Jiwoong Lee}

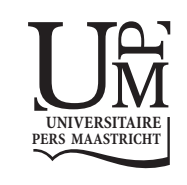


$1^{s t}$ Promotor:

Prof. dr. R.J. Müller

$2^{\text {nd }}$ Promotor:

Prof. dr. A.J. Vermeulen

Beoordelingscommissie:

Prof. dr. P. Jean-Jacques Herings (voorzitter)

Dr. János Flesch

Prof. dr. Dezsö Szalay (Universität Bonn) 


\section{Acknowledgements}

I owe a lot to a number of people.

My foremost thanks must go to my supervisors Rudolf Müller and Dries Vermeulen. They have shown immense patience and always helped me with their advice and guidance.

I cannot overstate my debt to Hans Peters. Without him, I could not have even started this dissertation.

I would like to extend my gratitude to the members of the committee, Jean-Jacques Herings, János Flesch and Dezsö Szalay, for taking their valuable time to read and referee this thesis. I am also grateful for Rakesh Vohra, with whom I have much benefited from several meetings.

Studying in the Netherlands and France, I received several financial assistance more than I deserve. I much appreciate the French ministry of education, the Europe-Korea Foundation and the Ilun Foundation for their support. In particular, this dissertation was made possible by financial support from the Netherlands Organisation for Scientific Research (NWO grant 400-09-190/MaGW).

I would like to thank my friends and colleagues in Maastricht University, who made these years a pleasant experience. Interacting with each one of them has been a true privilege. Especially, I am deeply indebted to my sincere friend and colleague Ehsan Valizadeh for his advice and insights he has passed on to me. 
Last but not least, I am grateful to my family. I thank Jinkyung for her love and support. I dedicate this dissertation work to my mother.

Jiwoong LEE

Maastricht, May 2013 


\section{Contents}

Acknowledgements

1 Introduction 1

1.1 Signaling Games . . . . . . . . . . . . . . . . . . . . . 1

1.2 Overview of the Dissertation $\ldots \ldots \ldots \ldots$

2 Separating Equilibria in One Dimensional Pure Signaling Games 5

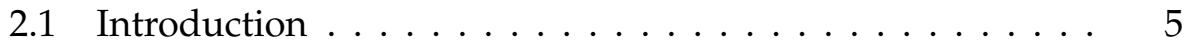

2.2 Model . . . . . . . . . . . . . . . . . . . 7

2.3 Graph representation of SE strategies . . . . . . . . . . . . . . 9

2.3.1 Characterization of SE strategies . . . . . . . . . . . . . 10

2.3.2 A few useful consequences .............. 11

2.4 Characterization of SE when $T$ is finite $\ldots \ldots \ldots \ldots 12$

2.4.1 Characterization . . . . . . . . . . . . . 12

2.4.2 Structure of the set of SE strategies . . . . . . . . . 13

2.4 .3 An algorithm . . . . . . . . . . . . . . . 15

2.5 Characterization of SE when $T$ is a continuum . . . . . . 17

2.5.1 Characterization . . . . . . . . . . . . . 18

2.5.2 Existence and Uniqueness of SE strategies . . . . . . . 21

2.6 Examples ....................... 22

2.6.1 Job market signaling . . . . . . . . . . . . . . 23

2.6.2 Matching under asymmetric information . . . . . 24

2.7 Conclusion . . . . . . . . . . . . . . . . . 26 


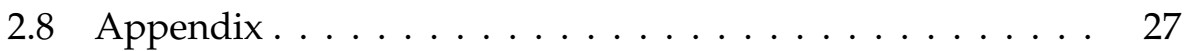

2.8.1 Riemann-Stieltjes integration . . . . . . . . . . . 27

2.8.2 Supplement to the Proof of Theorem 2.5.1 . . . . . . 29

2.8.3 Three Theorems Regarding the Initial Value Problem 32

3 Separating Equilibria in One Dimensional Non-Pure Signaling Games

3.1 Introduction . . . . . . . . . . . . . . . . 41

3.2 Model . . . . . . . . . . . . . . . . . . . . 43

3.2.1 Graph theoretic interpretation of SE . . . . . . . 46

3.3 Existence and Uniqueness of SE strategies . . . . . . . . . . . . . . . . . . . . . . . . . 51

3.4 Applications . . . . . . . . . . . . . 55

3.4.1 Signaling with a loss averse sender . . . . . . . . . . 56

3.4 .2 Security design . . . . . . . . . . . . . 59

3.5 Appendix........................... 63

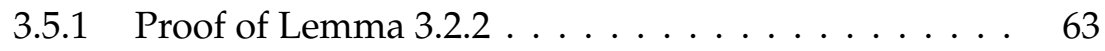

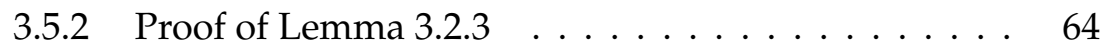

3.5.3 Two Theorems Regarding the Initial Value Problem . 65

4 Separating Equilibria in Multidimensional Signaling Games $\quad 71$

4.1 Introduction . . . . . . . . . . . . . . . . 71

4.2 Literature review . . . . . . . . . . . . . . . . 72

4.2.1 Unidimensional type, multidimensional signal cases 73

4.2.2 Multidimensional type, multidimensional signal cases 73

4.3 Model . . . . . . . . . . . . . . . . . . . . . . . . 74

4.3.1 Setup and notation . . . . . . . . . . . 74

4.3.2 Incentive Compatibility of Separating equilibrium . 77

4.4 Results . . . . . . . . . . . . . . . . . . 80

4.5 Discussion . . . . . . . . . . . . . . . . . 91

4.6 Conclusion . . . . . . . . . . . . . . . . . . . . 94

5 Voucher Privatization $\quad 95$

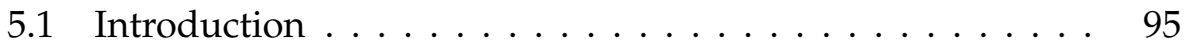

5.2 Model . . . . . . . . . . . . . . . . . . . . 97

5.2 .1 Timeline . . . . . . . . . . . . . . . . . . . . . . . . . . . 98

5.2 .2 Information structure . . . . . . . . . . . . 99 
5.2.3 Payoffs and Equilibrium . . . . . . . . . . . . . . . . . . . . 99

5.3 Analysis . . . . . . . . . . . . . . . 102

5.3 .1 Separating equilibrium . . . . . . . . . . . . . 102

5.3 .2 Pooling equilibrium ... . . . . . . . . 106

5.4 Comparison of Voucher Privatization and Direct Privatization111

5.4.1 $G$ 's Maximum revenue in direct privatization . . . 112

5.4 .2 Comparison .................. 114

5.5 Conclusion . . . . . . . . . . . . . . . . . . . . 116

$\begin{array}{ll}\text { Bibliography } & 117\end{array}$

$\begin{array}{ll}\text { Nederlandse samenvatting } & 121\end{array}$

$\begin{array}{ll}\text { Curriculum Vitae } & 123\end{array}$ 



\section{Chapter 1}

\section{Introduction}

\subsection{Signaling Games}

The theory of general equilibrium is one of the most important achievements in the history of economics. The proof of the existence of equilibrium, Pareto optimality, and the core seemed to pave the way for a reconstruction of economics around these concepts in the 1950s and 1960s. It turned out that the general equilibrium model was not a fully satisfactory descriptive tool, however.

The main reason is that strategic interactions between agents in an economy are almost absent in that model. In the general equilibrium model, agents only interact through the price system which they cannot influence by the pure competition assumption. Moreover, the organization of the many institutions that govern economic relationships is heavily constrained in the general equilibrium models. This is particularly striking in the case of firms because they are simply modeled as a production set. This makes the existence of firms hard to justify in the context of general equilibrium models, since all interactions are expected to take place through the price system in these models.

Another and equally serious challenge is that general equilibrium models fail to account for informational asymmetries. Asymmetries of information are pervasive in economic relationships. For example, firms do not know the exact tastes of customers, the government does not know 
firms' production technology, and all economic agents take actions that are not fully observable. To handle asymmetric information, rational expectations equilibria were conceived and presented interesting insights on the revelation of information by prices. But, their treatment of asymmetric information was not complete. ${ }^{1}$ Economic agents who possess private information, or type, will try to manipulate that information, since they have in fact a monopoly over their private information. To take this into account, we need to resort to other tools.

Information economics evolved from this failure of general equilibrium theory. In the 1970s several economists developed a new way to study economic relationships. The idea was to focus on partial models that consider the full complexity of strategic interactions between privately informed agents. The models of information economics are distinguished along several axes, depending on whether they are static or dynamic, whether they involve complete or incomplete contracts, whether they describe a bilateral or multilateral relationship, and so on. Here, I adopt the classification by Salanié (2005) who divides the models into the following three families:

- Signaling games. The uninformed party is imperfectly informed of the characteristics of the informed party. The informed party first moves.

- Adverse selection models. The informational environment is the same but the uninformed party first moves.

- Moral hazard models. The uninformed party first moves and is imperfectly informed of which action the informed party takes.

This dissertation studies the first family, signaling games. In these games, the informed party plays first by sending a signal that may reveal information relating to its type. The uninformed party then tries to decipher these signals by some endogeneously determined interpretative

\footnotetext{
${ }^{1}$ There are several studies which extend general equilibrium analysis to encompass a broad range of economic situations arising from asymmetric information in a perfectly competitive, general equilibrium framework. See, for example, Dubey et al. (2005) and Prescott and Townsend (1984).
} 
scheme. There are many equilibria in a signaling game, which can be classified into three different categories:

- Separating equilibria, where the signal chosen by the informed party identifies her type exactly.

- Pooling equilibria, where the observed signal conveys no additional information about the informed party's type.

- Semipooling equilibria, where some but not all information about the informed party's type is obtained from the observed signal.

When a separating equilibrium exists, then it is possible for the informed party to share her information fully with the uninformed party in spite of having a potential conflict of interest. Hence, a separating equilibrium is particularly of interest in the economic context and it is a benchmark outcome for signaling games (Sobel (2009)).

\subsection{Overview of the Dissertation}

In Chapters 2 to 4 , we provide a characterization of a separating equilibrium for standard signaling games where the sender's payoff function is quasilinear in the action taken by the receiver. Chapters 2 and 3 consider signaling games in which both type and signal spaces are single dimensional. Given a strategy of the sender, we construct a network where the node set and the length between two nodes are given as the set of the sender's type and the difference of signaling costs, respectively. It is observed that finding a separating equilibrium is equivalent to constructing the length between two nodes in the network under the condition that the response of the receiver is a node potential.

We show that, when the set of the sender's type is a real interval, the length of the shortest path in the network is antisymmetric and a node potential is unique up to constant. A strategy of the sender in a separating equilibrium turns out to be characterized by some differential equation with a unique solution. We also provide a set of sufficient conditions which guarantee the existence and uniqueness of separating equilibrium. 
Our result, relying on conditions which are milder than Mailath (1987) and Riley (1979) except for the quasilinearity, can be readily applied to a broad range of economic situations. In Chapter 2, we deal with the case in which the sender's payoff does not depend on the choice of signal. Chapter 3 allows for the possibility that the level of signal itself may influence the receiver's payoff.

Chapter 4 turns focus to signaling games in which both type and signal spaces are multidimensional. We show for a large class of cost functions that a sender's strategy constitutes a separating equilibrium if and only if it satisfies a certain set of conditions. In particular, we show that the sender's strategy in a separating equilibrium is given as a solution of a certain minimization problem. This is reminiscent of the derivation of the Hicksian demand function in traditional consumer theory. Our model is more general than Quinzii and Rochet (1985), who characterize a separating equilibrium in the case of separable, linear cost functions. We reconstruct some of their results by using our characterization.

In Chapter 5, we analyze voucher privatization from the perspective of signaling games. Russia in the early 1990s adopted a voucher auction to privatize state-owned companies. Despite of its wide use, this privatization scheme has received little attention. Restricting attention to the case that there is one firm to privatize, we characterize the set of voucher prices in equilibrium. We also compare voucher privatization with direct privatization by a government and conclude that voucher privatization may be an inferior scheme from the economic as well as political point of view. 


\section{Chapter 2}

\section{Separating Equilibria in One Dimensional Pure Signaling Games}

\subsection{Introduction}

A signaling game refers to a two-player game of incomplete information in which one player has private information, or a type, and the other is affected by the information. The informed player sends a signal, which may be contingent on her type, and the uninformed party takes an action, which may be conditional on the observed signal.

Signaling games arise in many economic settings. In the traditional example due to Spence (1973), a high-productivity job seeker invests in education to distinguish herself from low-productivity ones when productivity is not directly observed by an employer. In the context of industrial organization, a firm spends lots of money in advertising its newly launched product, of which quality is unknown to potential customers yet, on TV, newspapers and radio, which is sometimes much more than necessary to introduce the new product to the world. In auction theory, an auctioneer with private information about the object's quality puts a reserve price, taking a risk that an item in sale may not be sold in the end (Cai et al. (2007)). As an example from finance, when issuing a security backed by a 
fixed set of assets, an issuer who has private information about the assets is willing to retain some fraction of the issue though he prefers cash over the asset and so retention is costly (DeMarzo and Duffie (1999)). Examples are affluent and excellent surveys include Riley (2001) and Sobel (2009).

The privately informed player's choice of signal is assumed to have two distinct impacts on her payoff. The first is through the cost from sending the signal, and the second is through the inference the other player draws about the private information. In equilibrium, the informed player optimally chooses a signal, knowing that the uninformed player will take an optimal action conditional on his inferences from the signal and the inferences will turn out to be correct. If the inferences of the uninformed party leaves no uncertainty about the informed player's type, then the equilibrium is called separating. In a separating equilibrium, the informed party will deliver a type by type signal. Among many equilibria, a separating equilibrium is of central interest because it provides the possibility that informational asymmetry endogenously resolves. In this chapter, we will characterize a separating equilibrium in a pure signaling game with the real interval type space, where the word 'pure' means that the payoff of the uninformed party does not depend on a signal the informed party sends. The next chapter will relax the constraint so that non-pure games will be considered.

The papers closest related to ours are Mailath (1987) and Riley (1979). They consider signaling games in which private information is captured by an element in a real interval and provide a set of conditions under which the strategy of the informed party in separating equilibrium is differentiable. Though their results are quite strong and have been widely used in applications, some important environments are not covered by their results. We will provide and discuss such examples later in detail and show how our results can be used. We mention that a simultaneous and independent paper by Mailath and von Thadden (2013) extends the results of Mailath (1987) and their results can cover the examples we will discuss.

The contribution of this paper is two fold. First, an explicit representation of a separating equilibrium is given under conditions milder than those of Mailath (1987) and Riley (1979), assuming instead that the in- 
formed party's payoff function is quasi-linear in the uninformed party's action. This allows for the possibility that the payoff function has a kink due to, for instance, reference-dependent preferences as in Köszegi and Rabin (2006). Second, we provide a new interpretation of a separating equilibrium in terms of graph theory. Recently, graph theory has been successfully applied in mechanism design (e.g., Heydenreich et al. (2009), Mishra and Talman (2010) and Vohra (2011)). Ours is, to the best of our knowledge, the first to apply graph theory in signaling games.

The chapter is organized as follows. Section 2.2 presents the model of signaling games and Section 2.3 interprets a separating equilibrium in the view of graph theory. With this interpretation we characterize a separating equilibrium. Section 2.4 explores the case when private information is in a finite set of real numbers. We present a simple iterative algorithm to compute a separating equilibrium in this case. Section 2.5 deals with the case when private information is denoted as an element in a real interval. We characterize the informed party's strategy in separating equilibrium as a solution of a certain differential equation. In Section 2.6, we provide two examples from the literature which are not covered by the results of Mailath (1987) and show how our result can be applied to these examples. All omitted proofs are in Section 2.8.

\subsection{Model}

We consider the following standard signaling game. There are two risk neutral players, a sender and a receiver. The type space and the signal space of the sender are denoted by $T$ and $S$, respectively. We assume that the type space $T$ is a subset of $\mathbb{R}_{+} \equiv[0, \infty)$ and that the signal space $S$ equals $\mathbb{R}_{+}$. Further, $T$ is assumed to have a smallest element $\underline{t}$. The action space of the receiver is $A=\mathbb{R}_{+}$.

The game proceeds as follows. Nature decides the sender's type $t \in T$, and the result is communicated to the sender only. With knowledge of his type $t$, the sender chooses a signal $s \in S$, incurring a cost $c(s, t)$ depending on both type and signal. Upon observing the sender's signal $s$, the receiver chooses an action $a \in A$. The sender receives a payoff $U_{S}(a, s, t)$ and the 
Chapter 2. Separating Equilibria in One Dimensional Pure Signaling Games

receiver receives a payoff $U_{R}(a, t){ }^{1}$ Then the game ends.

A (pure) strategy of the sender is a function $\sigma: T \rightarrow S$. A (pure) strategy of the receiver is a function $\gamma: S \rightarrow A$. Since in this paper we only focus on pure strategies, we omit the prefix "pure" from now on.

Definition 2.2.1. A strategy pair $(\sigma, \gamma)$ is a separating equilibrium (SE) if the strategy $\sigma$ is one-to-one and, moreover, for all $t \in T$ we have

[1] $U_{S}(\gamma(\sigma(t)), \sigma(t), t) \geq U_{S}(\gamma(s), s, t) \quad$ for all $s \in S$

[2] $U_{R}(\gamma(\sigma(t)), t) \geq U_{R}(a, t)$ for all $a \in A$.

Condition [1] states that the strategy $\sigma$ is a best response for the sender given the strategy $\gamma$ of the receiver. Condition [2] states that the strategy $\gamma$ is a best response for the receiver given the strategy $\sigma$ of the sender.

If a strategy $\sigma$ of the sender is part of a separating equilibrium, it is called a separating equilibrium strategy (SE strategy). The aim of this chapter is to characterize the set of SE strategies ${ }^{2}$.

We assume that the sender's payoff is quasilinear in $a$, that is,

$$
U_{S}(a, s, t)=a-c(s, t)
$$

where $c$ is the signaling cost function.

Assumptions. We put the following structural assumptions on the payoff functions:

1. $c(s, t)$ is strictly increasing in $s$ for each $t$.

2. the cost function $c(s, t)$ has decreasing differences. That is, for all $s, s^{\prime} \in S$ and $t, t^{\prime} \in T$ with $s<s^{\prime}$ and $t<t^{\prime}$ it holds that

$$
c\left(s^{\prime}, t\right)-c(s, t)>c\left(s^{\prime}, t^{\prime}\right)-c\left(s, t^{\prime}\right) .
$$

\footnotetext{
${ }^{1}$ In this chapter, we assume that the receiver's payoff does not depend on signal. This is called the "pure signaling case" in Quinzii and Rochet (1985). We discuss the more general class of signaling games where the payoff of the receiver is also allowed to depend on the signal in Chapter 3.

${ }^{2}$ Note that the analogous task for the strategies of the receiver is a triviality.
} 
3. For every $t \in T$ there exists an $\alpha(t) \in A$ with

$$
U_{R}(\alpha(t), t)>U_{R}(a, t) \quad \text { for all } a \in A \backslash\{\alpha(t)\} .
$$

In other words, for every $t \in T, \alpha(t):=\underset{a \in A}{\arg \max _{R}} U_{R}(a, t)$ is unique.

4. $\alpha(\underline{t})=0$.

5. The resulting $\alpha$ is strictly increasing.

We end this section with a preliminary, but useful, observation.

Proposition 2.2.1. Let $(\sigma, \gamma)$ be an SE. Then $(\gamma \circ \sigma)(t)=\alpha(t)$ for all $t \in T$ if Assumption 3 holds. Further, $\sigma(\underline{t})=0$ if Assumption 1, 3 and 4 hold.

Proof. Let $(\sigma, \gamma)$ be an SE. By Assumption 3 and part [2] of the definition of SE, $(\gamma \circ \sigma)(t)=\alpha(t)$ for all $t \in T$.

Write $\underline{s}=\sigma(\underline{t})$. We show that $\underline{s}=0$. Suppose $\underline{s}>0$. Since $(\gamma \circ \sigma)(t)=\alpha(t)$ for all $t$, in particular $(\gamma \circ \sigma)(\underline{t})=\alpha(\underline{t})=0$ by Assumption 4. So,

$$
U_{S}(\gamma(\sigma(\underline{t})), \sigma(\underline{t}), \underline{t})=\gamma(\sigma(\underline{t}))-c(\sigma(\underline{t}), \underline{t})=0-c(\underline{s}, \underline{t}) .
$$

Since $S=\mathbb{R}_{+}$and $\underline{s}>0$ by assumption, we can take $0 \leq s<\underline{s}$. Then, since $\gamma(s) \in A=\mathbb{R}_{+}$and $c(s, \underline{t})$ is strictly increasing in $s$ (Assumption 1 ),

$$
U_{S}(\gamma(s), s, \underline{t})=\gamma(s)-c(s, \underline{t})>0-c(\underline{s}, \underline{t})=U_{S}(\gamma(\sigma(\underline{t})), \sigma(\underline{t}), \underline{t}) .
$$

This contradicts the assumption that $(\sigma, \gamma)$ is an SE. Hence, $\sigma(\underline{t})=\underline{s}=0$.

\subsection{Graph representation of SE strategies}

We provide a graph theoretic interpretation of SE strategies. We recall some basic terms from graph theory. A directed graph or digraph is a pair $\mathbf{G}=(V, E)$ where $V$ is an arbitrary set and $E$ is a collection of ordered pairs $(u, v)$ of elements $u, v \in V$ with $u \neq v$. The elements of $V$ are called nodes or vertices, and the elements of $E$ are called arcs. The complete digraph is the digraph $\mathbf{G}=(V, E)$ where $E$ is the set of all possible ordered pairs of distinct elements of $V$. 
Chapter 2. Separating Equilibria in One Dimensional Pure Signaling

Games

\subsubsection{Characterization of SE strategies}

Let $\mathbf{G}=(V, E)$ be a complete digraph. An arc length on $\mathbf{G}$ is a function $l: E \rightarrow \mathbb{R}$. A function $p: V \rightarrow \mathbb{R}$ is called a node potential for arc length $l$ on $\mathbf{G}=(V, E)$ if for all $\operatorname{arcs}(u, v) \in E$

$$
p(v)-p(u) \leq l(u, v) .
$$

Let $\sigma$ be a strategy of the sender. Let $\mathbf{H}=(T, Q)$ be the complete digraph on the type space $T$, so $Q=\{(u, v) \in T \times T \mid u \neq v\}$. Define the arc length $l_{\sigma}$ on $\mathbf{H}$ by, for all $t, t^{\prime} \in T$,

$$
l_{\sigma}\left(t, t^{\prime}\right)=c\left(\sigma\left(t^{\prime}\right), t\right)-c(\sigma(t), t)
$$

Theorem 2.3.1. Suppose that Assumptions 1, 3 and 4 hold. A strategy $\sigma$ of the sender is an SE strategy if and only if $\sigma$ is one-to-one, $\sigma(\underline{t})=0$, and $\alpha$ is a node potential for $l_{\sigma}$ on $\mathbf{H}$.

Proof. We show both implications separately.

$(\Longrightarrow) \quad$ Let $(\sigma, \gamma)$ be an SE. Since $\sigma$ is separating, it is one-to-one. Further, by Proposition 2.2.1, $\sigma(\underline{t})=0$. Also by Proposition 2.2.1, $(\gamma \circ \sigma)(t)=\alpha(t)$ for all $t$. Using [1] of the definition of separating equilibrium, for all $t$ and $s$

$$
\alpha(t)-c(\sigma(t), t)=(\gamma \circ \sigma)(t)-c(\sigma(t), t) \geq \gamma(s)-c(s, t) .
$$

Substitution of $s=\sigma\left(t^{\prime}\right)$ and rewriting then yields

$$
\alpha\left(t^{\prime}\right)-\alpha(t) \leq c\left(\sigma\left(t^{\prime}\right), t\right)-c(\sigma(t), t)=l_{\sigma}\left(t, t^{\prime}\right)
$$

for all $t, t^{\prime} \in T$. Hence, $\alpha$ is a node potential for $l_{\sigma}$ on $\mathbf{H}$.

$(\Leftarrow=) \quad$ Conversely, let $\sigma$ be a one-to-one strategy of the sender with $\sigma(\underline{t})=0$ and

$$
\alpha\left(t^{\prime}\right)-\alpha(t) \leq c\left(\sigma\left(t^{\prime}\right), t\right)-c(\sigma(t), t) .
$$

for all $t, t^{\prime} \in T$. Since $\sigma$ is one-to-one, for every $s \in S$ there is at most one $t \in T$ with $\sigma(t)=s$. So, we can define the strategy $\gamma$ of the receiver by

$$
\gamma(s)=\left\{\begin{aligned}
\alpha(t) & \text { if } t \in T \text { is such that } \sigma(t)=s \\
0 & \text { otherwise. }
\end{aligned}\right.
$$


It suffices to show that $(\sigma, \gamma)$ is a Nash equilibrium. First, for all $a \in A$

$$
U_{R}(\gamma(\sigma(t)), t)=U_{R}(\alpha(t), t) \geq U_{R}(a, t)
$$

by definition of $\gamma$ and $\alpha$. So, $\gamma$ is a best response of the receiver to $\sigma$.

Second, suppose the sender is of type $t$. Take $s \in S$. Suppose first there exists $t^{\prime} \in T$ with $\sigma\left(t^{\prime}\right)=s$. Then $\gamma(s)=\alpha\left(t^{\prime}\right)$. So, by assumption

$$
U_{S}(\gamma(\sigma(t)), \sigma(t), t)=\alpha(t)-c(\sigma(t), t) \geq \alpha\left(t^{\prime}\right)-c\left(\sigma\left(t^{\prime}\right), t\right)=U_{S}(\gamma(s), s, t)
$$

where the inequality is due to the assumption that $\alpha$ is a node potential.

Otherwise $\gamma(s)=0$. Then, we have

$$
\begin{aligned}
U_{S}(\gamma(\sigma(t)), \sigma(t), t) & =\alpha(t)-c(\sigma(t), t) & & \\
& \geq \alpha(\underline{t})-c(\sigma(\underline{t}), t) & & \text { (because } \alpha \text { is a node potential) } \\
& =0-c(0, t) & & \text { (by Assumption 4) } \\
& \geq \gamma(s)-c(s, t) & & \text { (by Assumption 1) } \\
& =U_{S}(\gamma(s), s, t) . & &
\end{aligned}
$$

Hence, $\sigma$ is a best response for the sender to $\gamma$.

\subsubsection{A few useful consequences}

We need a few direct consequences of the basic characterization in the remainder. We discuss these here in this section. One direct consequence of the above characterization is the following observation.

Proposition 2.3.1. Suppose that Assumptions 1 and 3-5 hold. If $\sigma$ is an SE strategy, then $\sigma$ is strictly increasing in $t$.

Proof. Take $t, t^{\prime} \in T$ with $t^{\prime}>t$. Since $\sigma$ is an SE strategy, by Theorem 2.3.1

$$
\alpha\left(t^{\prime}\right)-\alpha(t) \leq c\left(\sigma\left(t^{\prime}\right), t\right)-c(\sigma(t), t) .
$$

By Assumption 5 this implies $c\left(\sigma\left(t^{\prime}\right), t\right)>c(\sigma(t), t)$. Hence, since $c(s, t)$ is assumed to be strictly increasing in $s$ (Assumption 1), it follows that $\sigma\left(t^{\prime}\right)>$ $\sigma(t)$. 
Chapter 2. Separating Equilibria in One Dimensional Pure Signaling Games

An arc length $l$ is strictly monotone if $l\left(t, t^{\prime}\right)+l\left(t^{\prime}, t\right)>0$ for all $t \neq t^{\prime}$. The arc length $l$ is decomposition monotone if for any $t, t^{\prime}, t^{\prime \prime} \in T$ with $t<t^{\prime}<t^{\prime \prime}$ we have $l\left(t, t^{\prime \prime}\right)>l\left(t, t^{\prime}\right)+l\left(t^{\prime}, t^{\prime \prime}\right)$ and $l\left(t^{\prime \prime}, t\right)>l\left(t^{\prime \prime}, t^{\prime}\right)+l\left(t^{\prime}, t\right)$.

Lemma 2.3.1. Suppose $\sigma$ is strictly increasing and $c$ has decreasing differences (Assumption 2). Then arc length $l_{\sigma}$ is strictly monotone and decomposition monotone on $\mathbf{H}$.

Proof. We first show that $l_{\sigma}$ is strictly monotone. Take $t, t^{\prime}$ with $t<t^{\prime}$. Then $\sigma\left(t^{\prime}\right)>\sigma(t)$ by assumption. So, since $c$ has decreasing differences,

$$
l_{\sigma}\left(t, t^{\prime}\right)+l_{\sigma}\left(t^{\prime}, t\right)=c\left(\sigma\left(t^{\prime}\right), t\right)-c(\sigma(t), t)+c\left(\sigma(t), t^{\prime}\right)-c\left(\sigma\left(t^{\prime}\right), t^{\prime}\right)>0 .
$$

In order to prove that $l_{\sigma}$ is decomposition monotone, take $t, t^{\prime}, t^{\prime \prime} \in T$ with $t<t^{\prime}<t^{\prime \prime}$. Then, because $\sigma\left(t^{\prime \prime}\right)>\sigma\left(t^{\prime}\right)$ and $c$ has decreasing differences,

$$
\begin{aligned}
l_{\sigma}\left(t, t^{\prime \prime}\right) & =c\left(\sigma\left(t^{\prime \prime}\right), t\right)-c(\sigma(t), t) \\
& =c\left(\sigma\left(t^{\prime \prime}\right), t\right)-c\left(\sigma\left(t^{\prime}\right), t\right)+c\left(\sigma\left(t^{\prime}\right), t\right)-c(\sigma(t), t) \\
& >c\left(\sigma\left(t^{\prime \prime}\right), t^{\prime}\right)-c\left(\sigma\left(t^{\prime}\right), t^{\prime}\right)+c\left(\sigma\left(t^{\prime}\right), t\right)-c(\sigma(t), t) \\
& =l_{\sigma}\left(t^{\prime}, t^{\prime \prime}\right)+l_{\sigma}\left(t, t^{\prime}\right) .
\end{aligned}
$$

Similarly, we can derive that $l_{\sigma}\left(t^{\prime \prime}, t\right)>l_{\sigma}\left(t^{\prime}, t\right)+l_{\sigma}\left(t^{\prime \prime}, t^{\prime}\right)$. Hence, $l_{\sigma}$ is decomposition monotone.

\subsection{Characterization of SE when $T$ is finite}

The point of Theorem 2.3.1 is that finding a separating equilibrium strategy is equivalent to constructing an arc length for which $\alpha$ is a node potential on $\mathbf{H}$. We use this to characterize SE strategies.

\subsubsection{Characterization}

In this section we assume that $T=\left\{t_{1}, \ldots, t_{n}\right\}$ with $t_{i}<t_{i+1}$ for all $i=1, \ldots, n-$ 1. We first use Theorem 2.3.1 to provide a further characterization for this specific setting. Next, we use the resulting characterization to construct a separating equilibrium. Define

$$
R=\left\{\left(t_{k}, t_{k+1}\right) \mid k=1, \ldots, n-1\right\} \cup\left\{\left(t_{k+1}, t_{k}\right) \mid k=1, \ldots, n-1\right\} .
$$


Theorem 2.4.1. Suppose that Assumptions 1-5 hold. A strategy $\sigma$ of the sender is an SE strategy if and only if $\sigma$ is one-to-one, $\sigma(\underline{t})=0$, and $\alpha$ is a node potential for $l_{\sigma}$ on the restricted graph $(T, R)$.

Proof. The "only if" part is straightforward by Theorem 2.3.1. To show the "if" part, assume that $\alpha\left(t^{\prime}\right)-\alpha(t) \leq l_{\sigma}\left(t, t^{\prime}\right)$ for all $\left(t, t^{\prime}\right)$ in $R$. Take $\left(t_{k}, t_{k+i}\right) \in Q$ with $i \geq 2$. Since $\left(t_{k+j}, t_{k+j+1}\right) \in R$ for all $j=0, \ldots, i-1$,

$$
\alpha\left(t_{k+j+1}\right)-\alpha\left(t_{k+j}\right) \leq l_{\sigma}\left(t_{k+j}, t_{k+j+1}\right) .
$$

Therefore,

$$
\begin{aligned}
\alpha\left(t_{k+i}\right)-\alpha\left(t_{k}\right) & =\sum_{j=0}^{i-1}\left(\alpha\left(t_{k+j+1}\right)-\alpha\left(t_{k+j}\right)\right) \\
& \leq \sum_{j=0}^{i-1} l_{\sigma}\left(t_{k+j}, t_{k+j+1}\right) \\
& \leq l_{\sigma}\left(t_{k+i}, t_{k}\right) .
\end{aligned}
$$

The last inequality follows from decomposition monotonicity of $l_{\sigma}$, which in turn follows from Proposition 2.3.1, Assumption 2, and Lemma 2.3.1. By the same reasoning, we also have the node potential inequality for arcs $\left(t_{k+i}, t_{k}\right)$. Therefore, we have the desired result by Theorem 2.3.1.

\subsubsection{Structure of the set of SE strategies}

In this section we show that the set of SE strategies form a lattice. We use this fact in the next subsection to describe an elementary algorithm to check for existence of SE strategies.

Let $L$ denote the set of all non-decreasing sequences $\left(s_{1}, \ldots, s_{n}\right)$ in $S^{n}$ with $s_{1}=0$, and for all $k=1, \ldots, n-1$,

$$
c\left(s_{k+1}, t_{k+1}\right)-c\left(s_{k}, t_{k+1}\right) \leq \alpha\left(t_{k+1}\right)-\alpha\left(t_{k}\right) \leq c\left(s_{k+1}, t_{k}\right)-c\left(s_{k}, t_{k}\right) .
$$

Proposition 2.4.1. Suppose that Assumptions 1-5 hold. Let $\sigma$ be an SE strategy. Then $\left(\sigma\left(t_{1}\right), \ldots, \sigma\left(t_{n}\right)\right) \in L$. Conversely, let $s \in L$. Then $\sigma: t_{i} \mapsto s_{i}$ defines an SE strategy. 
Chapter 2. Separating Equilibria in One Dimensional Pure Signaling Games

Proof. Suppose that $s=\left(s_{1}, \ldots, s_{n}\right) \in L$. Define $\sigma: T \rightarrow S$ by $\sigma\left(t_{k}\right)=s_{k}$ for all $k$. Clearly, $\sigma(\underline{t})=0$. We show that $\sigma$ is strictly increasing (and hence one-to-one). Since $\alpha$ is assumed to be strictly increasing (Assumption 5), we have $\alpha\left(t_{k+1}\right)-\alpha\left(t_{k}\right)>0$. Therefore, since $s \in L$,

$$
c\left(s_{k+1}, t_{k+1}\right)-c\left(s_{k}, t_{k+1}\right)<c\left(s_{k+1}, t_{k}\right)-c\left(s_{k}, t_{k}\right)
$$

This implies $s_{k} \neq s_{k+1}$. Then, since $s \in L$, also $s_{k+1}>s_{k}$. Further,

$$
\begin{aligned}
\alpha\left(t_{k+1}\right)-\alpha\left(t_{k}\right) & \leq c\left(s_{k+1}, t_{k}\right)-c\left(s_{k}, t_{k}\right) \\
& =c\left(\sigma\left(t_{k+1}\right), t_{k}\right)-c\left(\sigma\left(t_{k}\right), t_{k}\right) \\
& =l_{\sigma}\left(t_{k}, t_{k+1}\right) .
\end{aligned}
$$

In the same way

$$
\alpha\left(t_{k}\right)-\alpha\left(t_{k+1}\right) \leq l_{\sigma}\left(t_{k+1}, t_{k}\right) .
$$

Hence, by Theorem 2.4.1, $\sigma$ is an SE strategy. The second claim follows directly from Proposition 2.3.1 and Theorem 2.3.1.

Thus, in the case where $T$ is finite, characterization of the collection of $\mathrm{SE}$ strategies reduces to characterization of the set $L$. This is our aim in the remainder of this section.

Since $L$ is a subset of $\mathbb{R}^{n}$, it is partially ordered by the usual coordinatewise order. For $x, y \in \mathbb{R}^{n}$, let $x \vee y \in \mathbb{R}^{n}$ be defined by $(x \vee y)_{i}=\max \left\{x_{i}, y_{i}\right\}$ and let $x \wedge y \in \mathbb{R}^{n}$ be defined by $(x \wedge y)_{i}=\min \left\{x_{i}, y_{i}\right\}$. A subset $X$ of $\mathbb{R}^{n}$ is called a lattice of $\mathbb{R}^{n}$ when for all $x, y \in X$ also $x \vee y \in X$ and $x \wedge y \in X$. We say that a lattice is lower bounded if it has a least element.

Theorem 2.4.2. Suppose that Assumptions 1-5 hold. The set $L$ is a closed and lower bounded lattice of $\mathbb{R}^{n}$.

Proof. Take two elements $s=\left(s_{1}, \ldots, s_{n}\right)$ and $r=\left(r_{1}, \ldots, r_{n}\right)$ in $L$. Define $x=s \wedge r$. Clearly $x$ is non-decreasing, and $x_{1}=0$. Take $k$. We show that

$$
c\left(x_{k+1}, t_{k+1}\right)-c\left(x_{k}, t_{k+1}\right) \leq \alpha\left(t_{k+1}\right)-\alpha\left(t_{k}\right) \leq c\left(x_{k+1}, t_{k}\right)-c\left(x_{k}, t_{k}\right) .
$$

If either $x_{k}=s_{k}$ and $x_{k+1}=s_{k+1}$, or if $x_{k}=r_{k}$ and $x_{k+1}=r_{k+1}$, this follows from the assumption that $s, r \in L$. Suppose that $x_{k}=s_{k}$ and $x_{k+1}=r_{k+1}$. 
Then we have

$$
\begin{aligned}
c\left(x_{k+1}, t_{k+1}\right)-c\left(x_{k}, t_{k+1}\right) & \leq c\left(s_{k+1}, t_{k+1}\right)-c\left(s_{k}, t_{k+1}\right) \\
& \leq \alpha\left(t_{k+1}\right)-\alpha\left(t_{k}\right),
\end{aligned}
$$

where the first inequality holds because $x_{k+1} \leq s_{k+1}, x_{k}=s_{k}$, and $c$ is strictly increasing s. Similarly,

$$
c\left(x_{k+1}, t_{k}\right)-c\left(x_{k}, t_{k}\right) \geq \alpha\left(t_{k+1}\right)-\alpha\left(t_{k}\right) .
$$

In the same way it can be shown that $s \vee r \in L$. Hence, $L$ is a lattice. Finally, $L$ is clearly closed, and, since $L$ is a subset of $\mathbb{R}_{+}^{n}$, also lower bounded.

\subsubsection{An algorithm}

We present a simple iterative procedure to compute an SE strategy in this setting. When $L$ is empty, the procedure will detect this and decide that there is no SE strategy.

We start by choosing $s_{1}=0$. Now suppose that $s_{k}$ is chosen. Choose $s_{k+1} \in S$ so that

$$
\alpha\left(t_{k+1}\right)-\alpha\left(t_{k}\right) \leq c\left(s_{k+1}, t_{k}\right)-c\left(s_{k}, t_{k}\right)
$$

and

$$
\alpha\left(t_{k+1}\right)-\alpha\left(t_{k}\right) \geq c\left(s_{k+1}, t_{k+1}\right)-c\left(s_{k}, t_{k+1}\right) .
$$

It is not always possible to choose such an $s_{k+1}$. However, as soon as such a choice is possible, automatically $s_{k+1}>s_{k}$ by the assumption of decreasing differences for the cost function $c(s, t)$. Thus, the procedure generates a sequence $s=\left(s_{1}, \ldots s_{n}\right)$, and by construction $s \in L$, unless at some point the above inequalities do not admit a feasible choice for $s_{k+1}$.

Example. Consider a signaling game with $T=\{1,2,3\}$ and cost function $c(s, t)=\frac{1}{t}\left(1-\frac{1}{s+1}\right)$. First consider the case where $(\alpha(1), \alpha(2), \alpha(3))=$ $(1,2,3)$. In this case there is no SE because the marginal cost for the sender is uniformly smaller than unity, while the marginal benefit by mimicking a higher type is unity, so that every type except the top has an incentive to mimick a higher type. Our algorithm states 
[1] $s_{1}=0$

[2] Choose $s_{2}>0$ such that $\frac{1}{2}\left(1-\frac{1}{s_{2}+1}\right) \leq 1 \leq\left(1-\frac{1}{s_{2}+1}\right)$.

[3] Choose $s_{3}>0$ such that $\frac{1}{3}\left(\frac{1}{s_{2}+1}-\frac{1}{s_{3}+1}\right) \leq 1 \leq \frac{1}{2}\left(\frac{1}{s_{2}+1}-\frac{1}{s_{3}+1}\right)$.

The condition in step 2 can be rewritten to $-1 \leq \frac{1}{s_{2}+1} \leq 0$, which is not feasible. Hence, the algorithm gets stuck in step 2 .

Now consider the case where $(\alpha(1), \alpha(2), \alpha(3))=(0,1 / 3,1 / 2)$. The algorithm states

[1] $s_{1}=0$

[2] Choose $s_{2}>0$ such that $\frac{1}{2}\left(1-\frac{1}{s_{2}+1}\right) \leq \frac{1}{3} \leq\left(1-\frac{1}{s_{2}+1}\right)$.

[3] Choose $s_{3}>0$ such that $\frac{1}{3}\left(\frac{1}{s_{2}+1}-\frac{1}{s_{3}+1}\right) \leq \frac{1}{6} \leq \frac{1}{2}\left(\frac{1}{s_{2}+1}-\frac{1}{s_{3}+1}\right)$, or $\frac{1}{s_{2}+1}-\frac{1}{2} \leq$ $\frac{1}{s_{3}+1} \leq \frac{1}{s_{2}+1}-\frac{1}{3}$.

The condition in step 2 states that $\frac{1}{3} \leq \frac{1}{s_{2}+1} \leq \frac{2}{3}$. If we choose $\frac{1}{s_{2}+1}=\frac{1}{3}, s_{3}$ must be chosen such that $1 /\left(s_{3}+1\right) \in[-1 / 6,0]$ in step 3 . Again, there does not exist such $s_{3}>0$ and the algorithm gets stuck.

We have an SE, though. For instance, the choice of $\frac{1}{s_{2}+1}=\frac{2}{3}$ in step 2 gives the feasible condition $1 /\left(s_{3}+1\right) \in[1 / 6,1 / 3]$ in step 3 .

Thus, in general our algorithm may get stuck, even though an SE strategy exists, and equivalently $L$ is not empty. We can avoid this irregularity by making an appropriate choice in each step of the algorithm. Since $L$ is a closed and lower bounded lattice, it has a minimum element as soon as $L$ is not empty. This minimum element of $L$ is the least costly SE strategy, and it is known as the Riley outcome. Our algorithm produces the Riley outcome if we consistently choose the lowest signal that satisfies the conditions, that is, the Riley outcome is obtained by choosing $s_{k+1}$ such that

$$
\alpha\left(t_{k+1}\right)-\alpha\left(t_{k}\right)=c\left(s_{k+1}, t_{k}\right)-c\left(s_{k}, t_{k}\right) .
$$

Finally we give a condition that guarantees existence of an SE strategy.

Assumption [E]. For all $t$, the cost function $c(s, t)$ is continuous in $s$, and $c(s, t) \rightarrow \infty$ as $s \rightarrow \infty$. 
Since $\alpha$ is strictly increasing, $c(s, t)$ is strictly increasing for all $t$, and $c(s, t)$ satisfies decreasing differences, by Assumption [E] such a choice as in (2.1) is feasible.

\subsection{Characterization of SE when $T$ is a continuum}

If the type space $T$ is a continuum, we can characterize a separating equilibrium strategy as a solution of a certain differential equation. The environment is the same as before except that the sender's type space $T$ is now an interval with the smallest element $\underline{t}$. Recall that both the signal space $S$ and the action space $A$ are $\mathbb{R}_{+}$.

Assumptions. We add the following assumptions on $c$ and $\alpha$.

6. The cost function $c(s, t)$ is continuous on $S \times T$.

7. The function $\alpha$ is continuous.

8. For all $t$, the partial derivative $c_{s}(s, t)$ of $c(s, t)$ with respect to $s$ exists and is continuous on $S \times T$.

Proposition 2.5.1. Suppose Assumptions 2 and 8 hold. Then the function $c_{s}(s, t)$ is non-increasing in $t$.

Proof. By Assumption 2, for $h>0$ and $t \leq t^{\prime}$,

$$
c\left(s+h, t^{\prime}\right)-c\left(s, t^{\prime}\right) \leq c(s+h, t)-c(s, t) .
$$

We can divide both sides by $h$ and take limits for $h \rightarrow 0$, which by Assumption 8 yields the inequality $c_{s}\left(s, t^{\prime}\right) \leq c_{s}(s, t)$.

We need the following observation in this setting.

Lemma 2.5.1. Suppose Assumptions 1-7 hold. Suppose that $\sigma$ is an SE strategy. Then, $\sigma$ is strictly increasing and continuous.

Proof. Let $(\sigma, \gamma)$ be an SE. Then $\sigma$ is strictly increasing by Proposition 2.3.1. We show that $\sigma$ is continuous. Take $t \in T$. Define $\sigma(t+):=\lim _{\varepsilon \downarrow 0} \sigma(t+\varepsilon)$. Since $(\sigma, \gamma)$ is an SE, for every sufficiently small $\varepsilon>0$ we have

$$
\gamma(\sigma(t+\varepsilon))-c(\sigma(t+\varepsilon), t+\varepsilon) \geq \gamma(\sigma(t))-c(\sigma(t), t+\varepsilon) .
$$


Chapter 2. Separating Equilibria in One Dimensional Pure Signaling Games

Since $\gamma(\sigma(t+\varepsilon))=\alpha(t+\varepsilon)$ and $\gamma(\sigma(t))=\alpha(t)$ by Proposition 2.2.1, this inequality implies that

$$
c(\sigma(t+\varepsilon), t+\varepsilon)-c(\sigma(t), t+\varepsilon) \leq \alpha(t+\varepsilon)-\alpha(t) .
$$

Since $c$ and $\alpha$ are assumed to be continuous (Assumptions 6 and 7), taking limits as $\varepsilon \downarrow 0$ yields

$$
c(\sigma(t+), t)-c(\sigma(t), t) \leq 0 .
$$

So, $\sigma(t+) \leq \sigma(t)$ by Assumption 1, which implies that $\sigma$ is right-continuous. Similarly, $\sigma$ is left-continuous. Hence, $\sigma$ is continuous.

\subsubsection{Characterization}

We characterize SE strategies for the sender. Before we state results, it is convenient to define the function $H: S \times \alpha(T) \rightarrow \mathbb{R}$ by

$$
H(s, a):=c_{s}\left(s, \alpha^{-1}(\alpha)\right),
$$

where $\alpha^{-1}$ exists on $\alpha(T)$ due to Assumption 5.

Theorem 2.5.1. Suppose Assumptions 1-8 hold.

1. If a strategy $\sigma$ is an SE strategy, then $\sigma=\gamma^{-1} \circ \alpha$, where $\gamma: S \rightarrow A$ is a partial solution on $\sigma(T)$ to the initial value problem

$$
\gamma^{\prime}(s)=H(s, \gamma(s)) \text { with } \gamma(0)=0 .
$$

2. Suppose $\gamma: S \rightarrow A$ is a solution to the initial value problem (DE) and $\alpha(T) \subseteq \gamma(S)$. Then, the function $\sigma=\gamma^{-1} \circ \alpha$ is an SE strategy,

Proof. 1. Let $\sigma$ be an SE strategy.

By Assumption 4 and Lemma 2.8.5 (see Appendix), it follows that

$$
\alpha(t)=\int_{\underline{t}}^{t} h_{\sigma}(x) d \sigma(x),
$$

where $h_{\sigma}(x)=c_{s}(\sigma(x), x)$.

By Lemma 2.5.1, $\sigma$ is strictly increasing and thus $\sigma^{-1}$ exists on $\sigma(T)$. Let $\gamma(s)=\left(\alpha \circ \sigma^{-1}\right)(s)$ for $s \in \sigma(T)$. Then $\gamma(0)=\alpha\left(\sigma^{-1}(0)\right)=\alpha(\underline{t})=0$ by Proposition 2.2.1 and Assumption 4. 
In addition, by Theorem 2.8.1, the change of variable $y=\sigma(x)$ yields for $s=\sigma(t)$ that

$$
\gamma(s)=\left(\alpha \circ \sigma^{-1}\right)(s)=\int_{\sigma(\underline{t})}^{\sigma(t)} c_{s}\left(y, \sigma^{-1}(y)\right) d y=\int_{0}^{s} H(y, \gamma(y)) d y .
$$

In the last equality we use $\sigma(\underline{t})=0$ by Proposition 2.2.1.

Since the function $y \mapsto H(y, \gamma(y))$ is continuous by Lemma 2.5.1 and Assumptions 7 and 8, the Fundamental Theorem of Calculus states that $\gamma$ is differentiable, and that for all $s \in \sigma(T)$

$$
\gamma^{\prime}(s)=H(s, \gamma(s))
$$

The desired result follows.

2. Let $\gamma: S \rightarrow A$ be a solution to the initial value problem

$$
\gamma^{\prime}(s)=H(s, \gamma(s)) \text { with } \gamma(0)=0 .
$$

Then $\gamma$ is differentiable and hence continuous. Further, $\gamma$ is also strictly increasing since $\gamma^{\prime}(s)=c_{s}\left(s, \alpha^{-1}(\gamma(s))\right)>0$ by Assumption 1 .

Since $\alpha(T) \subseteq \gamma(S)$, for every $t$ there exists an $s$ with $\gamma(s)=\alpha(t)$. So we can define $\sigma=\gamma^{-1} \circ \alpha$. Then $\sigma$ is also strictly increasing and continuous by Assumptions 5 and 7. Moreover, $\sigma(\underline{t})=\gamma^{-1}(\alpha(\underline{t}))=\gamma^{-1}(0)=0$. So, by Theorem 2.3.1 it suffices to show that $\alpha$ is a node potential for $l_{\sigma}$. Take $t, t^{\prime} \in T$ with $t \leq t^{\prime}$. We show that

$$
\alpha\left(t^{\prime}\right)-\alpha(t) \leq l_{\sigma}\left(t, t^{\prime}\right) .
$$

On the one hand, by definition of $\sigma$,

$$
\begin{aligned}
\alpha\left(t^{\prime}\right)-\alpha(t) & =(\gamma \circ \sigma)\left(t^{\prime}\right)-(\gamma \circ \sigma)(t)=\int_{\sigma(t)}^{\sigma\left(t^{\prime}\right)} \gamma^{\prime}(x) d x \\
& =\int_{\sigma(t)}^{\sigma\left(t^{\prime}\right)} H(x, \gamma(x)) d x=\int_{\sigma(t)}^{\sigma\left(t^{\prime}\right)} c_{s}\left(x, \alpha^{-1}(\gamma(x))\right) d x \\
& =\int_{\sigma(t)}^{\sigma\left(t^{\prime}\right)} c_{s}\left(x, \sigma^{-1}(x)\right) d x .
\end{aligned}
$$

On the other hand,

$$
l_{\sigma}\left(t, t^{\prime}\right)=c\left(\sigma\left(t^{\prime}\right), t\right)-c(\sigma(t), t)=\int_{\sigma(t)}^{\sigma\left(t^{\prime}\right)} c_{s}(x, t) d x .
$$


Chapter 2. Separating Equilibria in One Dimensional Pure Signaling Games

Now note that, since $\sigma$ is strictly increasing, for any $x \in\left[\sigma(t), \sigma\left(t^{\prime}\right)\right]$ we have $\sigma^{-1}(x) \geq t$. Therefore $c_{s}\left(x, \sigma^{-1}(x)\right) \leq c_{s}(x, t)$ for any $x \in\left[\sigma(t), \sigma\left(t^{\prime}\right)\right]$ by Proposition 2.5.1. Hence,

$$
\alpha\left(t^{\prime}\right)-\alpha(t)=\int_{\sigma(t)}^{\sigma\left(t^{\prime}\right)} c_{s}\left(x, \sigma^{-1}(x)\right) d x \leq \int_{\sigma(t)}^{\sigma\left(t^{\prime}\right)} c_{s}(x, t) d x=l_{\sigma}\left(t, t^{\prime}\right),
$$

as desired. We can apply the similar reasoning to the case when $t \geq t^{\prime}$.

Remark 2.5.1. The condition $\alpha(T) \subseteq \gamma(S)$ in the second claim of the theorem above matters. For illustration, consider the signaling game with $T=[1, \infty)$ and $\alpha(t)=2(t-1), c(s, t)=\frac{s}{t(s+1)}$. It is straightforward to verify that $c$ and $\alpha$ satisfy Assumptions 1-8. In this game, $H(s, a)=c_{s}\left(s, \alpha^{-1}(a)\right)=\frac{1}{\left(\frac{a}{2}+1\right)(s+1)^{2}}$ and thus the initial value problem (DE) becomes

$$
\gamma^{\prime}(s)=\frac{1}{\left(\frac{\gamma}{2}+1\right)(s+1)^{2}} \text { with } \gamma(0)=0 .
$$

The solution is $\gamma(s)=2\left(\sqrt{\frac{2 s+1}{s+1}}-1\right)$, which is smaller than $2(\sqrt{2}-1)$ for all $s$. Hence, the function $t \mapsto \gamma^{-1} \circ \alpha$ is not defined for $t$ such that $\alpha(t)>2(\sqrt{2}-1)$ and there is no separating equilibrium.

The reason for the failure of $S E$ is that the marginal signaling cost is upper bounded by $1 / t$ while the receiver's best action $\alpha$ is unbounded. To be more specific, suppose there is an SE. Choose $t$ and $t^{\prime}$ such that $\alpha\left(t^{\prime}\right)-\alpha(t)>1 / t$. This is possible due to the fact that $\alpha$ is unbounded. Then, the t-type sender has an incentive to mimic higher type $t^{\prime}$ because this deviation will strictly improve the sender's payoff as the increase in signaling cost, at most $1 / t$, is strictly lower than the increase in the receiver's response, $\alpha\left(t^{\prime}\right)-\alpha(t)$. This is a contradiction and hence there is no SE.

When $\alpha$ is differentiable, we can interpret the initial value problem (DE) in a more intuitive way. Suppose that $t$-type sender changes his signal by $\Delta s$. Then, the signaling cost will change by $c_{s} \Delta s$ while the level of action taken by the receiver will change by $\alpha^{\prime} \Delta t$. In equilibrium, to prevent any deviation it will hold that $c_{s} \Delta s=\alpha^{\prime} \Delta t$, that is,

$$
\frac{\Delta s}{\Delta t}=\frac{\alpha^{\prime}}{c_{s}} .
$$


On the other hand, under the differentiablity of $\alpha$, the derivative of $\gamma=\alpha \circ \sigma^{-1}$ is $\gamma^{\prime}=\left(\alpha^{\prime} \circ \sigma^{-1}\right) \cdot\left(\sigma^{-1}\right)^{\prime}$. We then write the initial value problem (DE) as

$$
\alpha^{\prime}\left(\sigma^{-1}(s)\right) \cdot\left(\sigma^{-1}\right)^{\prime}(s)=c_{s}\left(s, \sigma^{-1}(s)\right) .
$$

Setting $s=\sigma(t)$ yields $\sigma^{\prime}(t)=\frac{\alpha^{\prime}(t)}{c_{s}(\sigma(t), t)}$, which is essentially (2.2).

\subsubsection{Existence and Uniqueness of SE strategies}

As seen in Theorem 2.5.1, it is essential to solve the initial value problem (DE) in order to find an SE strategy. As such, the question naturally arises whether (DE) has a solution and, if so, whether it is unique. The following theorem provides two sets of necessary conditions for the existence and uniqueness, one for the case when the type space is a real half line, and one when the type space is compact.

Theorem 2.5.2. Suppose Assumptions 1-8 hold and there exists $M>0$ such that $c_{s}(s, t)<M$ for all $(s, t) \in S \times T$.

1. If $T=[\underline{t}, \infty)$ and $\alpha(t) \rightarrow \infty$ as $t \rightarrow \infty^{3}$, then the initial value problem (DE) has a unique solution $\gamma$. Moreover, if $\gamma \rightarrow \infty$ as $s \rightarrow \infty$, then $\sigma=\gamma^{-1} \circ \alpha$ is the unique SE strategy.

2. If $T=[\underline{t}, \bar{t}]$, then the initial value problem (DE) has a unique solution $\gamma$ on $\left[0, \frac{\alpha(\bar{t})}{M}\right]$. Moreover, if $\gamma\left(\frac{\alpha(\bar{t})}{M}\right) \geq \alpha(\bar{t})$, the function $\gamma^{-1} \circ \alpha: T \rightarrow S$ is the unique SE strategy.

Proof. We show the first claim. Since $\alpha^{-1}$ is defined on $A \equiv \mathbb{R}_{+}$because $\alpha(T)=\mathbb{R}_{+}$by assumption, the function $(s, a) \mapsto H(s, a)=c_{s}\left(s, \alpha^{-1}(a)\right)$ is defined on $S \times A=\mathbb{R}_{+} \times \mathbb{R}_{+}$. In addition, $H$ is bounded by assumption, continuous by Assumptions 7 and 8 , and non-increasing in the second argument due to Proposition 2.5.1. Thus, the initial value problem (DE) has a unique solution $\gamma$ by Theorems 2.8.2 and 2.8.4 (See Appendix). If $\gamma(s) \rightarrow \infty$ as $s \rightarrow \infty, \gamma^{-1} \circ \alpha$ is an SE strategy by the first claim of Theorem 2.5.1.

We claim uniqueness. If both $\sigma_{1}$ and $\sigma_{2}$ are SE strategies, two mappings $s \mapsto \alpha\left(\sigma_{1}^{-1}(s)\right)$ and $s \mapsto \alpha\left(\sigma_{2}^{-1}(s)\right)$ are a partial solutions on $\sigma_{1}(T)$ and

\footnotetext{
${ }^{3}$ In fact all we need is that for all $a \in A$ there exists a $t \in T$ with $\alpha(t)=a$
} 
$\sigma_{2}(T)$, respectively, of the same initial value problem (DE) by the first claim of Theorem 2.5.1. By Theorem 2.8.4, they coincide with each other, that is, $\alpha\left(\sigma_{1}^{-1}(s)\right)=\alpha\left(\sigma_{2}^{-1}(s)\right)$. Since $\alpha$ is assumed to be monotone (Assumption 5), $\sigma_{1}^{-1}(s)=\sigma_{2}^{-1}(s)$. The desired result follows.

We show the second claim. By assumption, the function $(s, a) \mapsto H(s, a)=$ $c_{s}\left(s, \alpha^{-1}(a)\right)$ is now defined on $S \times A=\mathbb{R}_{+} \times[0, \alpha(\bar{t})]$. Additionally, $H$ is bounded by assumption, continuous by Assumptions 7 and 8 , and nonincreasing in the second argument due to Proposition 2.5.1. Thus, the initial value problem (DE) has a unique solution $\gamma$ on $\left[0, \frac{\alpha(\bar{t})}{M}\right]$ by Theorems 2.8.3 and 2.8.4. If $\gamma\left(\frac{\alpha(\bar{t})}{M}\right) \geq \alpha(\bar{t})$, then $\gamma^{-1} \circ \alpha$ is an SE strategy by the second claim of Theorem 2.5.1. Uniqueness follows by the same reasoning as above.

\subsection{Examples}

In this section, we provide two examples of signaling games which do not satisfy the assumptions of Mailath (1987) but fall within the scope of our approach. The first is the continuous-type version of the classic job market model by Spence (1973). The second is a matching model with asymmetric information considered by Hoppe et al. (2009) in which agents are allowed to send signals to reveal their types.

For the sake of discussion, we recall the assumptions in Mailath (1987). To state them, it is convenient to define the reduced form payoff to a sender, $V: T \times T \times S \rightarrow \mathbb{R}$ as

$$
V\left(t, t^{\prime}, s\right)=U_{S}\left(\alpha\left(t^{\prime}\right), s, t\right)
$$

which is interpreted as the payoff of the type $t$ sender when he chooses signal $s$ and the receiver believes the sender to be of type $t^{\prime} .4$

Assuming that $T=\left[t_{0}, t_{1}\right], S=\mathbb{R}$ and $V$ is twice continuously differentiable, Mailath (1987) puts the following conditions on $V$.

C1. $s^{*}(t)=\arg \max _{s} V(t, t, s)$ uniquely exists for all $t$.

C2. For all $t \in\left(t_{0}, t_{1}\right), \frac{\partial^{2} V\left(t, t, s^{*}(t)\right)}{\partial s^{2}}<0$

\footnotetext{
${ }^{4}$ Mailath (1987) does not put the constraint that $U_{S}(a, s, t)$ is quasillinear in $a$ while we do.
} 
C3. There exists $k>0$ such that for all $(t, s) \in T \times S$

$$
\frac{\partial^{2} V(t, t, s)}{\partial s^{2}} \geq 0 \Longrightarrow\left|\frac{\partial V(t, t, s)}{\partial s}\right|>k
$$

C4. For all $\left(t, t^{\prime}, s\right) \in T^{2} \times S$,

$$
\frac{\partial^{2} V\left(t, t^{\prime}, s\right)}{\partial s \partial t} \neq 0 \text { and } \frac{\partial V\left(t, t^{\prime}, s\right)}{\partial t^{\prime}} \neq 0
$$

Under these conditions, Mailath (1987) shows that for a large class of signaling games, an SE strategy is differentiable and satisfies the standard differential equation.

Though his results are pioneering and quite general, the conditions unfortunately exclude some economic environments. Observe that condition [C2] excludes the case that $V$ is affine in $s$, that is, $V\left(t, t^{\prime}, s\right)=A\left(t, t^{\prime}\right)+B\left(t, t^{\prime}\right) s$ for some functions $A$ and $B$ on $T \times T$. However, this case often occurs in applications due to risk neutrality. In addition, the first constraint of condition [C4] also excludes the case that $V$ is additively separable with respect to $t$ and $s$, i.e., $V\left(t, t^{\prime}, s\right)=E\left(t, t^{\prime}\right)+F\left(t^{\prime}, s\right)$ for some functions $E$ and $F$ on $T \times T$ and $T \times S$, respectively. The following two examples from the literature do not satisfy either conditions [C2] or [C4]. The first violates [C2] while the second does both [C2] and [C4]. Using our results, we derive an SE strategy in each of the examples. We mention that the results in Mailath and von Thadden (2013), which extends Mailath (1987), can cover the examples.

\subsubsection{Job market signaling}

Spence (1973) is the seminal paper in signaling games. In his model, a job applicant has private information about his productivity, denoted as $t$. Because his productivity is not observed by an employer, the applicant tries to reveal it to an employer indirectly by investing in education, denoted as $s$. Since it is less costly for high types to receive the same level of education than for low types, there can be an equilibrium in which different types choose different signals. 
The structure of the signaling game under consideration is as follows: the sender and the receiver are the applicant and the employer, respectively, the type and signal sets are $T=[1,2]{ }^{5}$ and $S=\mathbb{R}_{+}$, respectively, and the best action of the receiver and the cost function of the sender are, respectively,

$$
\alpha(t)=t-1 \text { and } c(s, t)=\frac{s}{t} .
$$

In this setting, the reduced form payoff $V$ is given as $V\left(t, t^{\prime}, s\right)=t^{\prime}-1-\frac{s}{t}$ on $T \times T \times S$ and thus $V$ is affine in $s$ and thus the results of Mailath (1987) does not apply here.

However, the environment under consideration satisfies Assumptions 1-8 and so we can use our results, in particular Theorem 2.5.2. In the current setting, $H(s, a)=c_{s}\left(s, \alpha^{-1}(a)\right)=1 /(a+1)$ and the initial value problem $(\mathrm{DE})$ is

$$
\gamma^{\prime}(s)=\frac{1}{\gamma(s)+1} \text { with } \gamma(0)=0 .
$$

The unique solution to the above equation is $\gamma(s)=\sqrt{2 s+1}-1$. Because $\alpha(T)=[0,1] \subset[0, \infty)=\gamma(S)$, by the second claim of Theorem 2.5.2, the mapping $t \mapsto \gamma^{-1}(\alpha(t))=\frac{1}{2}\left(t^{2}-1\right)$ is the unique SE strategy.

\subsubsection{Matching under asymmetric information}

Signaling is also observed in situations where heterogeneous agents form matches in two-sided markets or in biological settings. On the one hand, signaling helps to maximize the total payoff generated from matching by determining who is matched with whom. On the other hand, this benefit can be offset by the signaling costs. Hoppe et al. (2009) provide a precise characterization and various consequences of this trade-off.

Hoppe et al. (2009) study the following two-sided matching contest. Each agent on both sides sends a costly signal $s \in S=\mathbb{R}_{+}$to a matchmaker and signals are delivered simultaneously. Agents on each side are ranked according to their signals and the matchmaker then matches them assortatively, that is, the agent with the highest signal in the one side is matched to the agent with the highest signal in the other side, etc. The payoff to

\footnotetext{
${ }^{5}$ In the original Spence model, $T=\{1,2\}$.
} 
an agent is the product of his and his partner's type minus the level of his signal.

No agent knows for sure his rank on his side nor the quality of a prospective equilibrium partner due to incomplete information. So, agents form expectations about the types of other agents on both sides of the market. Taking these expectations into consideration, Hoppe et al. (2009) derive the explicit expression $u(t)$ of the expected type of the partner whom an agent of type $t \in T=[1,2]$ is matched with. (For details, refer to Hoppe et al. (2009).) Thus, the expected payoff of an agent with type $t$ selecting a signal $s$ is written as

$$
V\left(t, t^{\prime}, s\right)=t \cdot u\left(t^{\prime}\right)-s,
$$

when the matchmaker believes the agent to be of type $t^{\prime}$. Notice that $V$ is not only affine in $s$ but also additively separable with respect to $t$ and $s$.

The problem of interest is to find an SE strategy $\sigma:[1,2] \rightarrow \mathbb{R}_{+}$which is strictly monotone and satisfies

$$
t \cdot u(t)-\sigma(t) \geq t \cdot u\left(t^{\prime}\right)-\sigma\left(t^{\prime}\right) \forall t, t^{\prime} \in[1,2]
$$

where $u$ is a strictly increasing and differentiable mapping with $u(1)=0$.

Hoppe et al. (2009) show that, assuming that an SE strategy is differentiable, ${ }^{6}$

$$
\sigma(t)=\int_{1}^{t} x \cdot u^{\prime}(x) d x
$$

is an SE strategy (Proposition 1 in Hoppe et al. (2009)). Using our result, we will see that their proposition can be strengthened further in two ways. First, dispensing with the assumption of differentiability, we can derive the same result. In this sense, they perform an analysis without loss of generality. Second, while their proposition states that such $\sigma$ is an SE strategy, it will in fact turn out to be the unique SE strategy.

The formal analysis is as follows. Let $T=[1,2], S=A=\mathbb{R}_{+}$and the best action of the receiver $\alpha(t)=u(t)$ and the cost function $c(s, t)=s / t$. Observe

\footnotetext{
${ }^{6}$ One can show that an SE strategy is absolutely continuous on [1,2] using (2.4) and so it is almost everywhere differentiable. In this sense, the assumption of differentiability is not so restricted.
} 
Chapter 2. Separating Equilibria in One Dimensional Pure Signaling Games

that (2.4) is equivalently rewritten as

$$
u(t)-\frac{\sigma(t)}{t} \geq u\left(t^{\prime}\right)-\frac{\sigma\left(t^{\prime}\right)}{t} \forall t, t^{\prime} \in[1,2]
$$

Suppose $\sigma$ is an SE strategy. Since the environment under consideration satisfies Assumptions 1-8, we can apply our results, in particular Lemma 2.8.5. In the current setting, we have $h_{\sigma}(x)=c_{s}(\sigma(x), x)=1 / x$ and

$$
u(t)-u\left(t^{\prime}\right)=\int_{t^{\prime}}^{t} \frac{1}{x} d \sigma(x)=\frac{\sigma(t)}{t}-\frac{\sigma\left(t^{\prime}\right)}{t^{\prime}}+\int_{t^{\prime}}^{t} \frac{\sigma(x)}{x^{2}} d x
$$

where the second equality comes from integration-by-parts.

Since the integrand $\frac{\sigma(x)}{x^{2}}$ is continuous by Lemma 2.5.1, $\int_{t^{\prime}}^{t} \frac{\sigma(x)}{x^{2}} d x$ is also differentiable with respect to $t$ by the fundamental theorem of calculus. In addition, because $u$ is differentiable, $\sigma$ is also differentiable in view of equality (2.6). Thus, we are able to differentiate both sides of (2.6) with respect to $t$, which gives

$$
u^{\prime}(t)=\frac{\sigma^{\prime}(t)}{t}
$$

which in turn implies

$$
\sigma(t)-\sigma\left(t^{\prime}\right)=\int_{t^{\prime}}^{t} x \cdot u^{\prime}(x) d x
$$

In addition, $\sigma(1)=0$ by Proposition 2.2.1. We thus obtain $\sigma(t)=\int_{1}^{t} x$. $u^{\prime}(x) d x$. Hence, if there is an SE strategy, it is uniquely given as $\sigma$. Lastly, it follows from Theorem 2.3.1 that $\sigma$ is indeed an SE strategy.

\subsection{Conclusion}

In this chapter, we provided a characterization of a separating equilibrium for standard pure signaling games where the sender's payoff function is quasilinear in the action taken by the receiver and both type and signal spaces are real intervals.

To this end, given a strategy of the sender, we constructed a network such that the node set and the length between two nodes are given as the set of the sender's types and the difference of signaling costs, respectively. 
We then observed that finding a separating equilibrium is equivalent to constructing the length between two nodes in the network such that the response of the receiver is a node potential. Based on this observation, we showed that a SE strategy can be characterized by some differential equation with a unique solution. We also provided a set of sufficient conditions which guarantee the existence and uniqueness of separating equilibrium. Finally, we presented two examples from the literature to which the results of Mailath (1987) are not applied but our results can be.

\subsection{Appendix}

\subsubsection{Riemann-Stieltjes integration}

We recall the concept of the Riemann-Stieltjes integral. For $r, t \in \mathbb{R}$ with $r<t$, a partition from $r$ to $t$ is a path $\pi=\left(t_{0}, \ldots, t_{k}\right)$ with

$$
r=t_{0}<t_{1}<\cdots<t_{k-1}<t_{k}=t .
$$

Let $P(r, t)$ be the collection of partitions from $r$ to $t$. Given a bounded function $f:[r, t] \rightarrow \mathbb{R}$ and a partition $\pi$ from $r$ to $t$, we define

$$
M_{i}=\sup \left\{f(t) \mid t_{i} \leq t \leq t_{i+1}\right\} \quad \text { and } \quad m_{i}=\inf \left\{f(t) \mid t_{i} \leq t \leq t_{i+1}\right\} .
$$

Let $g$ be non-decreasing on $[r, t]$. Given a partition $\pi$ from $r$ to $t$ we write $\Delta g_{i}=g\left(t_{i+1}\right)-g\left(t_{i}\right)$. Write

$$
U(\pi, f, g)=\sum_{i=0}^{k-1} M_{i} \cdot \Delta g_{i} \quad \text { and } \quad L(\pi, f, g)=\sum_{i=0}^{k-1} m_{i} \cdot \Delta g_{i}
$$

It is straightforward to check that $\inf _{\pi \in P(r, t)} U(\pi, f, g)$ and $\sup _{\pi \in P(r, t)} L(\pi, f, g)$ exist, and that

$$
\inf _{\pi \in P(r, t)} U(\pi, f, g) \geq \sup _{\pi \in P(r, t)} L(\pi, f, g) .
$$

When

$$
\inf _{\pi \in P(r, t)} U(\pi, f, g)=\sup _{\pi \in P(r, t)} L(\pi, f, g)
$$


Chapter 2. Separating Equilibria in One Dimensional Pure Signaling Games

we denote their common value by $\int_{r}^{t} f(x) d g(x)$. This value is called the Riemann-Stieltjes integral of $f$ with respect to $g$ on $[r, t]$. It is known that $\int_{r}^{t} f(x) d g(x)$ exists whenever $f$ is continuous ${ }^{7}$.

For $r, t \in T$ with $r>t$, a partition from $r$ to $t$ is a path $\pi=\left(t_{0}, \ldots, t_{k}\right)$ with

$$
r=t_{0}>t_{1}>\cdots>t_{k-1}>t_{k}=t .
$$

Let $P(r, t)$ be the collection of partitions from $r$ to $t$. We define $M_{i}, m_{i}$ and $\Delta g_{i}$ as before, but now

$$
L(\pi, f, g)=\sum_{i=0}^{k-1} M_{i} \cdot \Delta g_{i} \quad \text { and } \quad U(\pi, f, g)=\sum_{i=0}^{k-1} m_{i} \cdot \Delta g_{i}
$$

Again, it is straightforward to check that $\inf _{\pi \in P(r, t)} U(\pi, f, g)$ and $\sup _{\pi \in P(r, t)} L(\pi, f, g)$ exist, and that

$$
\inf _{\pi \in P(r, t)} U(\pi, f, g) \geq \sup _{\pi \in P(r, t)} L(\pi, f, g) .
$$

When

$$
\inf _{\pi \in P(r, t)} U(\pi, f, g)=\sup _{\pi \in P(r, t)} L(\pi, f, g),
$$

we denote their common value by $\int_{t}^{r} f(x) d g(x)$. It is straightforward to check that

$$
\int_{r}^{t} f(x) d g(x)=-\int_{t}^{r} f(x) d g(x) .
$$

In principle, this is equivalent to a definition of the Riemann-Stieltjes integral when $g$ is non-increasing.

The following theorem will be used later. For its proof, see Rudin (1976), p.133.

Theorem 2.8.1. Let $\phi:[A, B] \rightarrow[a, b]$ be strictly increasing and continuous. Let $\alpha$ be an increasing function on $[a, b]$. Let $f$ be Riemann-Stieltjes integrable on $[a, b]$ w.r.t. $\alpha$. For $y \in[A, B], \beta(y)=\alpha(\phi(y))$ and $g(y)=f(\phi(y))$. Then $g$ is Riemann-Stieltjes integrable w.r.t. $g$, and

$$
\int_{A}^{B} g(y) d \beta(y)=\int_{a}^{b} f(x) d \alpha(x) .
$$

\footnotetext{
${ }^{7}$ See Rudin (1976), p.125.
} 


\subsubsection{Supplement to the Proof of Theorem 2.5.1}

This section is devoted to supplementing the proof of Theorem 2.5.1. To this end, we need the following notions. Consider a complete digraph $\mathbf{G}=(V, E)$ on a finite set $V$ of nodes. A path is a vector $\pi=\left(v_{0}, \ldots, v_{k}\right)$ with $\left(v_{i}, v_{i+1}\right) \in E$ for all $i=0, \ldots, k-1$. For two nodes $u, v \in V$, a path from $u$ to $v$ is a path $\left(v_{0}, \ldots, v_{k}\right)$ with $v_{0}=u$ and $v_{k}=v$. For $r, t \in V$, the set of all paths from $r$ to $t$ is denoted by $\Pi(r, t)$.

Let $l$ be an arc length on $\mathbf{G}$. The length of a path $\pi=\left(v_{0}, \ldots, v_{k}\right)$, denoted by length $(l)(\pi)$, is defined as

$$
\text { length }(l)(\pi)=\sum_{i=0}^{k-1} l\left(v_{i}, v_{i+1}\right) .
$$

The distance from $r$ to $t$ with respect to the the arc length $l$ is defined by

$$
\operatorname{dist}(r, t)=\inf _{\pi \in \Pi(r, t)} \operatorname{length}(l)(\pi) .
$$

We now consider the graph $\mathbf{H}$ defined in Section 2.3 and let $\sigma$ be a continuous and strictly increasing strategy for the sender. We write as $\operatorname{dist}_{\sigma}\left(t, t^{\prime}\right)$ the distance from $t$ to $t^{\prime}$ with respect to the the arc length $l_{\sigma}$.

Lemma 2.8.1. Suppose that $\sigma$ is strictly increasing and $c$ has decreasing differences (Assumption 2). Then for any $t, t^{\prime} \in T$ with $r \neq t$,

$$
\operatorname{dist}_{\sigma}\left(t, t^{\prime}\right)=\inf _{\pi \in P\left(t, t^{\prime}\right)} \operatorname{length}\left(l_{\sigma}\right)(\pi) .
$$

Proof. We prove the statement in case $t<t^{\prime}$. The proof for the case where $t>t^{\prime}$ runs along similar lines. By definition of dist it is obvious that

$$
\operatorname{dist}_{\sigma}\left(t, t^{\prime}\right) \leq \inf _{\pi \in P\left(t, t^{\prime}\right)} l_{\sigma}(\pi) .
$$

To show the reverse inequality, it suffices to prove the following claim: for any path $\pi \in \Pi\left(t, t^{\prime}\right)$ there exists a partition $\rho \in P\left(t, t^{\prime}\right)$ with $l_{\sigma}(\rho) \leq l_{\sigma}(\pi)$.

To prove the claim take any path $\pi=\left(t_{0}, \ldots, t_{n}\right)$ in $\mathbf{H}$ from $t$ to $t^{\prime}$. If there is any edge $\left(t_{k}, t_{k+1}\right)$ such that there exists an $i$ with $t_{k}<t_{i}<t_{k+1}$ or $t_{k+1}<t_{i}<t_{k}$ define a new path $\rho=\left(t_{0}, \ldots, t_{k}, t_{i}, t_{k+1}, \ldots, t_{n}\right)$. By decomposition monotonicity, $l_{\sigma}(\rho) \leq l_{\sigma}(\pi)$. Replace $\pi$ by $\rho$ and iterate this procedure until no such edges can be found. At this point, for all $k$, with 
Chapter 2. Separating Equilibria in One Dimensional Pure Signaling Games

$t_{k} \leq t_{k+1}$, either $t_{k+2} \geq t_{k+1}$, or $t_{k+2}=t_{k}$. In the second case define a new path $\rho=\left(t_{0}, t_{k}, t_{k+3}, t_{n}\right)$. Note that the set of edges of $\rho$ is a subset of the set of edges of $\pi$, as $t_{k+2}=t_{k}$, and that by monotonicity the two eliminated edges have to together a non-positive length. Therefore, $l_{\sigma}(\rho) \leq l_{\sigma}(\pi)$.

Iterating this procedure yields either a path running through the nodes of a partition $P\left(t, t^{\prime}\right)$ from $t$ in direction $t^{\prime}$, or a path with $t_{0}>t_{1}$. In the later case, there must exist a $k$ such that $t_{k}>t_{k+1}$ and $t_{k+2}=t_{k}$, as $t<t^{\prime}$. In this case we may again replace $\pi$ by $\rho=\left(t_{0}, t_{k}, t_{k+3}, t_{n}\right)$ yielding a path that is no longer than before. Iterating also this sequence of eliminations yields a path running through the nodes of a partition $P\left(t, t^{\prime}\right)$ from $t$ in direction $t^{\prime}$. The length of this path is no longer than the length of the original path $\pi$.

Lemma 2.8.2. Suppose Assumptions 2 and 8 hold. If $\sigma$ is continuous and strictly increasing, then for any partition $\pi$ from $r$ to $t$,

$$
L\left(\pi, h_{\sigma}, \sigma\right) \leq \operatorname{length}\left(l_{\sigma}\right)(\pi) \leq U\left(\pi, h_{\sigma}, \sigma\right),
$$

where the function $h_{\sigma}: T \rightarrow \mathbb{R}$ is defined by $h_{\sigma}(t)=c_{s}(\sigma(t), t)$

Proof. We construct the proof for the case where $r<t$. Again, the remaining case where $r>t$ runs along similar lines. Take a partition $\pi=\left(t_{0}, \ldots, t_{k}\right)$ from $r$ to $t$. Take $i$ fixed. By Assumption 8, the map $y \mapsto c_{s}\left(y, t_{i}\right)$ is continuous on the interval $\left[\sigma\left(t_{i}\right), \sigma\left(t_{i+1}\right)\right]$. Write $m_{i}=\min \left\{c_{s}\left(y, t_{i}\right) \mid \sigma\left(t_{i}\right) \leq y \leq\right.$ $\left.\sigma\left(t_{i+1}\right)\right\}$. Now, since $\sigma$ is strictly increasing,

$$
\begin{aligned}
\operatorname{length}\left(l_{\sigma}\right)(\pi) & =\sum_{i=0}^{k-1} l_{\sigma}\left(t_{i}, t_{i+1}\right) \\
& =\sum_{i=0}^{k-1}\left[c\left(\sigma\left(t_{i+1}\right), t_{i}\right)-c\left(\sigma\left(t_{i}\right), t_{i}\right)\right] \\
& \geq \sum_{i=0}^{k-1} m_{i} \cdot\left[\sigma\left(t_{i+1}\right)-\sigma\left(t_{i}\right)\right] .
\end{aligned}
$$

On the other hand,

$$
L(\pi, h, \sigma)=\sum_{i=0}^{k-1} n_{i} \cdot\left[\sigma\left(t_{i+1}\right)-\sigma\left(t_{i}\right)\right]
$$


where $n_{i}=\min \left\{c_{s}(\sigma(x), x) \mid t_{i} \leq x \leq t_{i+1}\right\}$. By Proposition 2.5.1, it holds that $c_{s}(\sigma(x), x) \leq c_{s}\left(\sigma(x), t_{i}\right)$. Since $\sigma$ is continuous and strictly increasing, it follows that

$$
\begin{aligned}
m_{i} & =\min \left\{c_{s}\left(y, t_{i}\right) \mid \sigma\left(t_{i}\right) \leq y \leq \sigma\left(t_{i+1}\right)\right\} \\
& =\min \left\{c_{s}\left(\sigma(x), t_{i}\right) \mid t_{i} \leq x \leq t_{i+1}\right\} \\
& \geq n_{i}
\end{aligned}
$$

Hence, length $\left(l_{\sigma}\right)(\pi) \geq L\left(\pi, h_{\sigma}, \sigma\right)$. Similarly, length $\left(l_{\sigma}\right)(\pi) \leq U\left(\pi, h_{\sigma}, \sigma\right)$.

For two partitions $\pi=\left(t_{1}, \ldots, t_{k}\right)$ and $\rho=\left(r_{1}, \ldots, r_{m}\right)$, we write $\pi \vee \rho$ for the (unique) partition $\left(u_{1}, \ldots, u_{p}\right)$ with

$$
\left\{u_{1}, \ldots, u_{p}\right\}=\left\{r_{1}, \ldots, r_{m}\right\} \cup\left\{t_{1}, \ldots, t_{k}\right\} .
$$

Lemma 2.8.3. Suppose Assumptions 2 and 8 hold. If $\sigma$ is strictly increasing and continuous, then

$$
\sup _{\pi \in P(r, t)} L\left(\pi, h_{\sigma}, \sigma\right) \leq \operatorname{dist}_{\sigma}(r, t) .
$$

Proof. By Lemma 2.3.1, $l_{\sigma}$ is decomposition monotone. So, length $\left(l_{\sigma}\right)(\pi \mathrm{V}$ $\rho) \leq$ length $\left(l_{\sigma}\right)(\pi)$ for any $\pi, \rho \in P(r, t)$. Since also $L\left(\pi, h_{\sigma}, \sigma\right) \leq L\left(\pi \vee \rho, h_{\sigma}, \sigma\right)$ for any $\pi, \rho \in P(r, t)$, we get the result by Lemma 2.8.2.

Lemma 2.8.4. Suppose Assumptions 2 and 8 hold. If $\sigma$ is strictly increasing and continuous, then for all $r, t \in T$ with $r \neq t$,

$$
\int_{r}^{t} h_{\sigma}(x) d \sigma(x)=\operatorname{dist}_{\sigma}(r, t) .
$$

Proof. Observe first that the function $h_{\sigma}$ is continuous in $t$ by Assumption 8. Hence, the Riemann-Stieltjes integral $\int_{r}^{t} h_{\sigma}(x) d \sigma(x)$ exists for all $r, t \in T$ with $r \neq t$.

Then, by Lemmas 2.8.2 and 2.8.1,

$$
\int_{r}^{t} h_{\sigma}(x) d \sigma(x)=\inf _{\pi \in P(r, t)} U\left(\pi, h_{\sigma}, \sigma\right) \geq \inf _{\pi \in P(r, t)} \operatorname{length}\left(l_{\sigma}\right)(\pi)=\operatorname{dist}_{\sigma}(r, t) .
$$


Chapter 2. Separating Equilibria in One Dimensional Pure Signaling Games

On the other hand, by Lemma 2.8.3,

$$
\int_{r}^{t} h_{\sigma}(x) d \sigma(x)=\sup _{\pi \in P(r, t)} L\left(\pi, h_{\sigma}, \sigma\right) \leq \operatorname{dist}_{\sigma}(r, t) .
$$

This completes the proof.

Lemma 2.8.5. Suppose Assumptions 1-4 and 8 hold. If $\sigma$ is an SE strategy, then for all $r, t \in T$,

$$
\alpha(t)-\alpha(r)=\int_{r}^{t} h_{\sigma}(x) d \sigma(x) .
$$

Proof. Take $r, t$ with $r \neq t$. Let $\pi=\left(t_{0}, \ldots, t_{k}\right)$ be a partition from $r$ to $t$. Then

$$
\begin{aligned}
\alpha(t)-\alpha(r) & =\sum_{i=0}^{k-1}\left(\alpha\left(t_{i+1}\right)-\alpha\left(t_{i}\right)\right) \\
& \leq \sum_{i=0}^{k-1} l_{\sigma}\left(t_{i}, t_{i+1}\right) \\
& =\text { length }\left(l_{\sigma}\right)(\pi)
\end{aligned}
$$

where the inequality is due to Theorem 2.3.1.

Hence, by Lemma 2.8.1 and Lemma 2.8.4

$$
\alpha(t)-\alpha(r) \leq \inf _{\pi \in P(r, t)} \operatorname{length}\left(l_{\sigma}\right)(\pi)=\operatorname{dist}_{\sigma}(r, t)=\int_{r}^{t} h_{\sigma}(x) d \sigma(x) .
$$

This also implies that

$$
\alpha(r)-\alpha(t) \leq \int_{t}^{r} h_{\sigma}(x) d \sigma(x)=-\int_{r}^{t} h_{\sigma}(x) d \sigma(x)
$$

so that

$$
\int_{r}^{t} h_{\sigma}(x) d \sigma(x) \leq \alpha(t)-\alpha(r)
$$

This completes the proof.

\subsubsection{Three Theorems Regarding the Initial Value Problem}

We show three general facts which are relevant to the initial value problem (DE). Theorems 2.8.2 and 2.8.3 below address the existence of a solution while Theorem 2.8.4 is about uniqueness of a solution. The proofs of Theorems 2.8.2 and 2.8.3 are loosely based on Peano's Theorem (See p.10 in Hartman (1982) for example). 
Theorem 2.8.2. Let $V=\mathbb{R}_{+} \times \mathbb{R}_{+}$. Suppose $H: V \rightarrow \mathbb{R}_{+}$is continuous and bounded. Then there exists at least one solution of the initial value problem

$$
f^{\prime}(s)=H(s, f(s)) \text { with } f(0)=0 .
$$

Moreover, any solution is automatically continuously differentiable.

Proof. For $n, k \in \mathbb{N}$ define $s_{n, k}=k \cdot 2^{-n}$. Now take $n \in \mathbb{N}$ fixed for the moment. We set $x_{n, 0}=0$, and for $k \in \mathbb{N}$

$$
x_{n, k+1}=x_{n, k}+2^{-n} \cdot H\left(s_{n, k}, x_{n, k}\right) .
$$

Define $f_{n}: \mathbb{R}_{+} \rightarrow \mathbb{R}$ by, for $s \in\left[s_{n, k}, s_{n, k+1}\right)$,

$$
f_{n}(s)=x_{n, k}+2^{n} \cdot\left(s-s_{n, k}\right) \cdot\left(x_{n, k+1}-x_{n, k}\right) .
$$

Let $M>0$ be such that $|H(s, a)| \leq M$ for all $(s, a) \in V$. First we show for all $s, s^{\prime} \in \mathbb{R}_{+}$that

$$
\left|f_{n}\left(s^{\prime}\right)-f_{n}(s)\right| \leq M \cdot\left|s^{\prime}-s\right| .
$$

Take $k, m$ such that $s \in\left[s_{n, k}, s_{n, k+1}\right)$ and $s^{\prime} \in\left[s_{n, m}, s_{n, m+1}\right)$. Then $f_{n}(s)$ is a linear interpolation between $x_{n, k}$ and $x_{n, k+1}$, and $f_{n}\left(s^{\prime}\right)$ is a linear interpolation between $x_{n, m}$ and $x_{n, m+1}$. Therefore it suffices to prove that

$$
\left|x_{n, k+1}-x_{n, k}\right| \leq M \cdot\left|s_{n, k+1}-s_{n, k}\right| .
$$

This however immediately follows from the observation that

$$
\begin{aligned}
\left|x_{n, k+1}-x_{n, k}\right| & =\left|2^{-n} \cdot H\left(s_{n, k}, x_{n, k}\right)\right| \\
& \leq 2^{-n} \cdot M \\
& =M \cdot\left|s_{n, k+1}-s_{n, k}\right| .
\end{aligned}
$$

Now write

$$
D=\left\{k \cdot 2^{-n} \mid k, n \in \mathbb{N}\right\} .
$$

Obviously $D$ is a countable set. Let $d_{1}, d_{2}, \ldots$ be an enumeration of $D$. Consider $d_{1} \in D$. Then for all $n$ we have $\left|f_{n}\left(d_{1}\right)\right| \leq M \cdot d_{1}$. So, there is a subsequence $\left(f_{n}^{1}\right)_{n=1}^{\infty}$ of $\left(f_{n}\right)_{n=1}^{\infty}$ for which $\left(f_{n}^{1}\left(d_{1}\right)\right)_{n=1}^{\infty}$ is convergent. Iteratively for $k=1,2, \ldots$ we can take a subsequence $\left(f_{n}^{k}\right)_{n=1}^{\infty}$ of $\left(f_{n}^{k-1}\right)_{n=1}^{\infty}$ for which 
Chapter 2. Separating Equilibria in One Dimensional Pure Signaling Games

$\left(f_{n}^{k}\left(d_{l}\right)\right)_{n=1}^{\infty}$ is convergent for all $l=1, \ldots k$. Then for the sequence $\left(f_{k}^{k}\right)_{k=1}^{\infty}$ we have that $\left(f_{k}^{k}(d)\right)_{k=1}^{\infty}$ is convergent for all $d \in D$.

Write $g_{k}=f_{k}^{k}$. Take any $s \geq 0$. We argue that $\left(g_{k}(s)\right)_{k=1}^{\infty}$ is Cauchy. Take $\varepsilon>0$. Since $D$ is dense in $\mathbb{R}_{+}$, we can take a $d \in D$ with $|s-d|<\frac{\varepsilon}{3 M}$. Further, since $\left(g_{k}(d)\right)_{k=1}^{\infty}$ is convergent, we can take $K>0$ such that

$$
\left|g_{k}(d)-g_{m}(d)\right|<\frac{\varepsilon}{3}
$$

whenever $k, m>K$. Then, for $k, m>K$,

$$
\begin{aligned}
\left|g_{k}(s)-g_{m}(s)\right| & =\left|g_{k}(s)-g_{k}(d)+g_{k}(d)-g_{m}(d)+g_{m}(d)-g_{m}(s)\right| \\
& \leq\left|g_{k}(s)-g_{k}(d)\right|+\left|g_{k}(d)-g_{m}(d)\right|+\left|g_{m}(d)-g_{m}(s)\right| \\
& <M \cdot|s-d|+\frac{\varepsilon}{3}+M \cdot|s-d| \\
& \leq M \cdot \frac{\varepsilon}{3 M}+\frac{\varepsilon}{3}+M \cdot \frac{\varepsilon}{3 M}=\varepsilon .
\end{aligned}
$$

Define $f(s)=\lim _{n \rightarrow \infty} g_{n}(s)$. We claim that $f$ is continuous. Take any $s, s^{\prime} \geq 0$. Since $\left(g_{n}\right)_{n=1}^{\infty}$ is a subsequence of $\left(f_{n}\right)_{n=1}^{\infty}$, we know from the argument above that

$$
\left|g_{n}\left(s^{\prime}\right)-g_{n}(s)\right| \leq M \cdot\left|s^{\prime}-s\right|
$$

for all $n$. The claim now follows by taking limits for $n \rightarrow \infty$.

So, the function $s \mapsto H(s, f(s))$ is continuous. Then it is integrable on bounded intervals. Take any $s \geq 0$. We show that

$$
f(s)=\int_{0}^{s} H(u, f(u)) d u .
$$

Take $n \in \mathbb{N}$ fixed. Let $p(n)$ be the natural number such that $g_{n}=f_{n}^{n}=f_{p(n)}$. Define

$$
h_{n}(s)=H\left(s_{p(n), k}, x_{p(n), k}\right) \quad \text { if } s \in\left[s_{p(n), k}, s_{p(n), k+1}\right) .
$$

Then clearly $g_{n}(s)=\int_{0}^{s} h_{n}(u) d y$. We argue that $h_{n}(s) \rightarrow H(s, f(s))$ as $n \rightarrow \infty$. First note that

$$
h_{n}\left(s_{p(n), k}\right)=H\left(s_{p(n), k}, x_{p(n), k}\right)=H\left(s_{p(n), k}, f_{p(n)}\left(s_{p(n), k}\right)\right)=H\left(s_{p(n), k}, g_{n}\left(s_{p(n), k}\right)\right) .
$$


Take $s \geq 0$ arbitrary. Choose $k(n)$ such that $s \in\left[s_{p(n), k(n)}, s_{p(n), k(n)+1}\right)$. Then

$$
h_{n}(s)=H\left(s_{p(n), k(n)}, x_{p(n), k(n)}\right)=H\left(s_{p(n), k(n)}, g_{n}\left(s_{p(n), k(n)}\right)\right) .
$$

Since clearly $p(n) \rightarrow \infty$ as $n \rightarrow \infty$, we know that $s_{p(n), k(n)} \rightarrow s$ as $n \rightarrow \infty$. Therefore it suffices to show that

$$
g_{n}\left(s_{p(n), k(n)}\right) \rightarrow f(s) .
$$

This however follows from the observations that $g_{n}(s) \rightarrow f(s)$ and $\mid g_{n}\left(s^{\prime}\right)-$ $g_{n}(s)|\leq M \cdot| s^{\prime}-s \mid$.

Hence, since $\left|h_{n}(s)\right| \leq M$ for all $s$ and $n$, the theorem of bounded convergence yields

$$
f(s)=\lim _{n \rightarrow \infty} g_{n}(s)=\lim _{n \rightarrow \infty} \int_{0}^{s} h_{n}(u) d u=\int_{0}^{s} H(u, f(u)) d u .
$$

This completes the proof.

Theorem 2.8.3. Let $V=\mathbb{R}_{+} \times[0, \bar{a}]$. Suppose $H: V \rightarrow \mathbb{R}_{+}$is continuous and bounded and denote by $M$ the supreme of $H$. Then there exists at least one solution of the initial value problem

$$
f^{\prime}(s)=H(s, f(s)) \text { with } f(0)=0 .
$$

for $s \in\left[0, \frac{\bar{a}}{M}\right]$. Moreover, any solution is automatically continuously differentiable.

The proof is almost the same as in 2.8.2.

Proof. Let $n \in \mathbb{N}$ and $k \in\left\{0,1, \ldots,\left\lfloor 2^{n} \bar{a} / M\right\rfloor\right\}$, where $\lfloor z\rfloor$ is the largest integer not greater than $z$. Define $s_{n, k}=k \cdot 2^{-n}$.

Now take $n \in \mathbb{N}$ fixed for the moment. We set $x_{n, 0}=0$ and

$$
x_{n, k+1}=x_{n, k}+2^{-n} \cdot H\left(s_{n, k}, x_{n, k}\right),
$$

for $k=0,1, \ldots,\left\lfloor 2^{n} \bar{a} / M\right\rfloor-1$. Notice that (2.7) is well-defined because

$$
\begin{aligned}
x_{n, k} & =x_{n, 0}+\sum_{i=0}^{k-1} 2^{-n} H\left(s_{n, i}, x_{n, i}\right) \\
& \leq 0+2^{-n} \cdot(k-1) \cdot M \\
& <2^{-n} \cdot 2^{n} \frac{\bar{a}}{M} \cdot M=\bar{a}
\end{aligned}
$$


Chapter 2. Separating Equilibria in One Dimensional Pure Signaling

Games

Define $f_{n}:\left[0, \frac{\bar{a}}{M}\right] \rightarrow \mathbb{R}$ by

$$
f_{n}(s)=\left\{\begin{array}{cl}
x_{n, k}+2^{n}\left(x_{n, k+1}-x_{n, k}\right)\left(s-s_{n, k}\right) & \text { if } s \in\left[s_{n, k}, s_{n, k+1}\right) \\
x_{\left.n, \frac{2^{n} \bar{a}}{M}\right\rfloor}+2^{n}\left(x_{n,\left\lfloor\frac{2^{n}}{M}\right\rfloor}-x_{n,\left\lfloor\frac{2^{n} \bar{a}}{M}\right\rfloor-1}\right)\left(s-s_{n,\left\lfloor\frac{2^{n} \bar{a}}{M}\right\rfloor}\right) & \text { if } s \in\left[s_{n,\left\lfloor\frac{2^{n} \bar{a}}{M}\right\rfloor}, \frac{\bar{a}}{M}\right]
\end{array}\right.
$$

First we show for all $s, s^{\prime} \in\left[0, \frac{\bar{a}}{M}\right]$ that

$$
\left|f_{n}\left(s^{\prime}\right)-f_{n}(s)\right| \leq M \cdot\left|s^{\prime}-s\right|
$$

Case 1. If $s \in\left[s_{n, k}, s_{n, k+1}\right)$ and $s^{\prime} \in\left[s_{n, m}, s_{n, m+1}\right)$ for some $k, m$, then $f_{n}(s)$ is a linear interpolation between $x_{n, k}$ and $x_{n, k+1}$, and $f_{n}\left(s^{\prime}\right)$ is a linear interpolation between $x_{n, m}$ and $x_{n, m+1}$. Therefore it suffices to prove that

$$
\left|x_{n, k+i}-x_{n, k}\right| \leq M \cdot\left|s_{n, k+i}-s_{n, k}\right| .
$$

This however immediately follows from the observation that

$$
\begin{aligned}
\left|x_{n, k+1}-x_{n, k}\right| & =\left|2^{-n} \cdot H\left(s_{n, k}, x_{n, k}\right)\right| \\
& \leq 2^{-n} \cdot M \\
& =M \cdot\left|s_{n, k+1}-s_{n, k}\right| .
\end{aligned}
$$

Case 2. If $s^{\prime}, s \in\left[s_{n,\left\lfloor\frac{2^{n_{\bar{a}}}}{M}\right\rfloor}, \frac{\bar{a}}{M}\right]$, then

$$
\begin{aligned}
\left|f_{n}\left(s^{\prime}\right)-f_{n}(s)\right| & =\left|2^{n}\left(x_{n,\left\lfloor\frac{\left.2^{n_{\bar{a}}}\right\rfloor}{M}\right\rfloor}-x_{n,\left\lfloor\frac{2^{n_{\bar{a}}}}{M}\right\rfloor-1}\right)\left(s^{\prime}-s\right)\right| \\
& =\left|2^{n} \cdot 2^{-n} H\left(s_{n,\left\lfloor\frac{2^{n} \bar{a}}{M}\right\rfloor-1}, x_{n,\left\lfloor\frac{2^{n} \bar{a}}{M}\right\rfloor-1}\right)\right| \cdot\left|s^{\prime}-s\right| \\
& \leq M\left|s^{\prime}-s\right| .
\end{aligned}
$$


Case 3. If $s^{\prime} \in\left[s_{n,\left\lfloor\frac{2^{n}}{M}\right\rfloor}, \frac{\bar{a}}{M}\right]$ and $s \in\left[s_{n, k}, s_{n, k+1}\right)$, then

$$
\begin{aligned}
\left|f_{n}\left(s^{\prime}\right)-f_{n}(s)\right|= & \left|f_{n}\left(s^{\prime}\right)-f\left(s_{n,\left\lfloor\frac{2^{n} \bar{a}}{M}\right\rfloor}\right)+f\left(s_{n, 2^{n_{\bar{a}}}}\right)-f\left(s_{n, k+1}\right)+f\left(s_{n, k+1}\right)-f_{n}(s)\right| \\
= & 2^{n}\left(x_{n,\left\lfloor\frac{2^{n} \bar{a}}{M}\right\rfloor}-x_{n,\left\lfloor\frac{2^{n} \bar{a}}{M}\right\rfloor-1}\right)\left(s^{\prime}-s_{n,\left\lfloor\frac{2^{n} \overline{\bar{a}}}{M}\right\rfloor}\right) \\
& +f\left(s_{n,\left\lfloor\frac{2^{n} \bar{a}}{M}\right\rfloor}\right)-f\left(s_{n, k+1}\right) \\
& \quad+2^{n}\left(x_{n, k+1}-x_{n, k}\right)\left(s_{n, k+1}-s\right) \\
\leq & 2^{n} \cdot 2^{-n} M \cdot\left(s^{\prime}-s_{n, 2^{n_{\bar{a}}}}\right) \\
& +2^{n} \cdot 2^{-n} M \cdot\left(s_{n,\left\lfloor\frac{2^{n} \overline{\bar{a}}}{M}\right\rfloor}-s_{n, k+1}\right) \\
& \quad+2^{n} \cdot 2^{-n} M \cdot\left(s_{n, k+1}-s\right) \\
= & M \cdot\left|s^{\prime}-s\right| \\
\leq & M\left|s^{\prime}-s\right| .
\end{aligned}
$$

Hence, (2.8) follows as desired.

Now write

$$
D=\left\{k \cdot 2^{-n} \mid n \in \mathbb{N}, k=0,1, \ldots,\left\lfloor 2^{n} \bar{a} / M\right\rfloor\right\} .
$$

Obviously $D$ is a countable set. Let $d_{1}, d_{2}, \ldots$ be an enumeration of $D$. Consider $d_{1} \in D$. Then for all $n$ we have $\left|f_{n}\left(d_{1}\right)\right| \leq M \cdot d_{1}$. So, there is a subsequence $\left(f_{n}^{1}\right)_{n=1}^{\infty}$ of $\left(f_{n}\right)_{n=1}^{\infty}$ for which $\left(f_{n}^{1}\left(d_{1}\right)\right)_{n=1}^{\infty}$ is convergent. Iteratively for $m=1,2, \ldots$ we can take a subsequence $\left(f_{n}^{m}\right)_{n=1}^{\infty}$ of $\left(f_{n}^{m-1}\right)_{n=1}^{\infty}$ for which $\left(f_{n}^{m}\left(d_{l}\right)\right)_{n=1}^{\infty}$ is convergent for all $l=1, \ldots m$. Then for the sequence $\left(f_{m}^{m}\right)_{m=1}^{\infty}$ we have that $\left(f_{m}^{m}(d)\right)_{m=1}^{\infty}$ is convergent for all $d \in D$.

Write $g_{m}=f_{m}^{m}$. Take any $s \in\left[0, \frac{\bar{a}}{M}\right]$. We argue that $\left(g_{m}(s)\right)_{k=1}^{\infty}$ is Cauchy. Take $\varepsilon>0$. Since $D$ is dense in $\mathbb{R}_{+}$, we can take a $d \in D$ with $|s-d|<\varepsilon / 3 M$. Further, since $\left(g_{m}(d)\right)_{m=1}^{\infty}$ is convergent, we can take $K>0$ such that

$$
\left|g_{m}(d)-g_{m^{\prime}}(d)\right|<\frac{\varepsilon}{3}
$$

whenever $m, m^{\prime}>K$. Then, for $m, m^{\prime}>K$,

$$
\begin{aligned}
\left|g_{m}(s)-g_{m^{\prime}}(s)\right| & =\left|g_{m}(s)-g_{m}(d)+g_{m}(d)-g_{m^{\prime}}(d)+g_{m^{\prime}}(d)-g_{m^{\prime}}(s)\right| \\
& \leq\left|g_{m}(s)-g_{m}(d)\right|+\left|g_{m}(d)-g_{m^{\prime}}(d)\right|+\left|g_{m^{\prime}}(d)-g_{m^{\prime}}(s)\right| \\
& <M \cdot|s-d|+\frac{\varepsilon}{3}+M \cdot|s-d| \\
& \leq M \cdot \frac{\varepsilon}{3 M}+\frac{\varepsilon}{3}+M \cdot \frac{\varepsilon}{3 M}=\varepsilon .
\end{aligned}
$$


Chapter 2. Separating Equilibria in One Dimensional Pure Signaling Games

Define $f(s)=\lim _{m \rightarrow \infty} g_{m}(s)$. We claim that $f$ is continuous. Take any $s, s^{\prime} \geq 0$. Since $\left(g_{m}\right)_{m=1}^{\infty}$ is a subsequence of $\left(f_{m}\right)_{m=1}^{\infty}$, we know from the argument above that

$$
\left|g_{m}\left(s^{\prime}\right)-g_{m}(s)\right| \leq M \cdot\left|s^{\prime}-s\right|
$$

for all $m$. The claim now follows by taking limits for $m \rightarrow \infty$.

So, the function $s \mapsto H(s, f(s))$ is continuous. Then it is integrable on bounded intervals. Take any $s \geq 0$. We show that

$$
f(s)=\int_{0}^{s} H(u, f(u)) d u .
$$

Take $n \in \mathbb{N}$ fixed. Let $p(n)$ be the natural number such that $g_{n}=f_{n}^{n}=f_{p(n)}$. Define

$$
h_{n}(s)=H\left(s_{p(n), k}, x_{p(n), k}\right) \quad \text { if } s \in\left[s_{p(n), k}, s_{p(n), k+1}\right) .
$$

Then clearly $g_{n}(s)=\int_{0}^{s} h_{n}(u) d y$. We argue that $h_{n}(s) \rightarrow H(s, f(s))$ as $n \rightarrow \infty$. First note that

$h_{n}\left(s_{p(n), k}\right)=H\left(s_{p(n), k}, x_{p(n), k}\right)=H\left(s_{p(n), k}, f_{p(n)}\left(s_{p(n), k}\right)\right)=H\left(s_{p(n), k}, g_{n}\left(s_{p(n), k}\right)\right)$.

Take $s \geq 0$ arbitrary. Choose $k(n)$ such that $s \in\left[s_{p(n), k(n)}, s_{p(n), k(n)+1}\right)$. Then

$$
h_{n}(s)=H\left(s_{p(n), k(n)}, x_{p(n), k(n)}\right)=H\left(s_{p(n), k(n)}, g_{n}\left(s_{p(n), k(n)}\right)\right) .
$$

Since clearly $p(n) \rightarrow \infty$ as $n \rightarrow \infty$, we know that $s_{p(n), k(n)} \rightarrow s$ as $n \rightarrow \infty$. Therefore it suffices to show that

$$
g_{n}\left(s_{p(n), k(n)}\right) \rightarrow f(s) .
$$

This however follows from the observations that $g_{n}(s) \rightarrow f(s)$ and $\mid g_{n}\left(s^{\prime}\right)-$ $g_{n}(s)|\leq M \cdot| s^{\prime}-s \mid$.

Hence, since $\left|h_{n}(s)\right| \leq M$ for all $s$ and $n$, the theorem of bounded convergence yields

$$
f(s)=\lim _{n \rightarrow \infty} g_{n}(s)=\lim _{n \rightarrow \infty} \int_{0}^{s} h_{n}(u) d u=\int_{0}^{s} H(u, f(u)) d u .
$$

This completes the proof. 
A subset $I$ of $S$ is called an initial subset if for all $s \in I$ and $s^{\prime} \in S$ with $s^{\prime}<s$ we have $s^{\prime} \in I$. A function $g: I \rightarrow \mathbb{R}$ is called a partial solution on $I$ if $g(0)=0, g$ is differentiable on $I$, and

$$
g^{\prime}(s)=H(s, g(s))
$$

for all $s \in I$.

Theorem 2.8.4. Let $V=\mathbb{R}_{+} \times \mathbb{R}_{+}$or $V=\mathbb{R}_{+} \times[0, \bar{a}]$. Suppose that $H: V \rightarrow \mathbb{R}$ is continuous and non-increasing in the second coordinate. Then the initial value problem

$$
f^{\prime}(s)=H(s, f(s)) \text { with } f(0)=0
$$

has at most one solution $f$. Moreover, if $f$ is the solution, for any partial solution $g$ on $I$ it holds that $f(s)=g(s)$ for all $s \in I$.

Proof. Suppose that $f$ is a solution of the initial value problem, and that $g$ is a partial solution on $I$. It suffices to show that $f(s)=g(s)$ for all $s \in I$.

For $s \in I$, define $h(s)=f(s)-g(s)$. We show that $h(s)=0$ for all $s \in I$. Suppose that $h(s)>0$ for some $s \in I$. Since $h(0)=0$, obviously $s>0$. Define

$$
s^{*}=\inf \{x \mid 0 \leq x \leq s \text { and } h(y)>0 \text { for all } y \in(x, s)\} .
$$

Since $h$ is continuous, $h(s)>0$, and $h(0)=0$, we know that $s^{*}<s$ and $h\left(s^{*}\right)=$ 0 . So, since $h$ is continuously differentiable, by the mean value theorem there exists $\tau \in\left(s^{*}, s\right)$ with

$$
h^{\prime}(\tau)=\frac{h(s)-h\left(s^{*}\right)}{s-s^{*}}=\frac{h(s)}{s-s^{*}}>0 .
$$

However, $\tau \in\left(s^{*}, s\right)$ implies that $h(\tau)>0$ by definition of $s^{*}$. Then $f(\tau)>$ $g(\tau)$. So, since $H$ is non-increasing in the second argument, $f^{\prime}(\tau)=H(\tau, f(\tau)) \leq$ $H(\tau, g(\tau))=g^{\prime}(\tau)$. Hence, $h^{\prime}(\tau) \leq 0$. Contradiction. 



\section{Chapter 3}

\section{Separating Equilibria in One Dimensional Non-Pure Signaling Games}

\subsection{Introduction}

In this chapter, we consider an extension of the signaling game discussed in the previous chapter. In some applications of signaling games, the level of signal itself may influence the receiver's payoff. For instance, in auction theory the level of reserve price sometimes not only can play the role of signal to convey private information of an auctioneer (sender) about an auctioned object but also affect the strategy of a bidder (receiver), resulting in change in a bidder's final payoff (Cai et al. (2007) and Jullien and Mariotti (2006)).

We present a characterization of an SE strategy and investigate its existence and uniqueness in a class of signaling games which allow the receiver's payoff to be dependent on signal as well as his own action and the sender's type. We mention that the paper by Mailath (1987) and its recent extension by Mailath and von Thadden (2013) study this issue thoroughly and provide fairly general results. Instead, in this chapter we are interested in situations where the application of their results may fail due to the absence of differentiability assumptions on the sender's payoff func- 
Chapter 3. Separating Equilibria in One Dimensional Non-Pure Signaling Games

tion, which is crucial in their results. The following simple example serves as an illustration in which the differentiability assumption is not valid.

Example 3.1.1 (Loss averse sender). We consider a signaling game with a sender having reference-dependent preferences modeled by Köszegi and Rabin (2006). Let the best response of the receiver be $\alpha(s, t) \in A$ when $s \in S$ is a signal chosen by the sender and $t \in T$ is the sender's type, where $A, S$ and $T$ are real intervals. The sender has a reference point $r \in A$ and assesses deviations of the receiver's action $a \in A$ from $r$ in a different way, depending on whether they are gains or losses. As Köszegi and Rabin suggest, the sender's payoff $\hat{U}_{S}: A \times S \times T \rightarrow \mathbb{R}$ is written as

$$
\hat{U}_{S}(a, s, t)=\left\{\begin{array}{cl}
a-c(s, t) & \text { if } a \leq r \\
a+k(a-r)-c(s, t) & \text { otherwise. }
\end{array}\right.
$$

where $k>0$ is a constant and $c$ is the signaling cost function. Clearly, the payoff function is not differentiable. We denote by $\mathscr{G}$ this signaling game for future reference.

The problem in the above example is caused by the kink in the sender's payoff. One may argue that the above example should be treated as a mathematical anomaly, which can be safely ignored in applications. However, as Carbajal and Ely (2013) argue convincingly, because kinks in the sender's payoff function may capture economic relevant components, they should be taken into account in many behavioral models. This kind of behavioral models includes, in addition to the model of reference-dependent preferences, the disappointment aversion model by Gul (1991) and the inequality aversion model by Fehr and Schmidt (1999).

In this chapter, we will see that an SE strategy is closely related to a solution to the initial value problem. This result is somewhat similar to what we have seen in the previous chapter. We will also present a set of conditions under which the initial value problem has a unique solution. We remark that our results are based on the assumption that the sender's payoff is quasilinear in the receiver's action while Mailath (1987) and Mailath and von Thadden (2013) do not assume such quasilinearity. Hence, if a sender is risk averse, for instance, their results should be used. On the other hand, our results require a much weaker differentiability condition than theirs. 
Thus, if the sender's payoff has kinks, say, because a sender is loss averse, our results are applicable. In this sense, the results in this chapter and those from Mailath (1987) and Mailath and von Thadden (2013) complement each other.

The remaining of chapter is organized as follows. Section 3.2 presents the model of extended signaling games and interprets a separating equilibrium in the view of graph theory. With this interpretation, in section 3.3 we address existence and uniqueness of SE strategies. In Section 3.4, we show how our results can be applied to two examples.

\subsection{Model}

We consider the standard signaling game described in the previous chapter. There are two risk neutral players, a sender and a receiver. The type space and the signal space of the sender are denoted by $T$ and $S$, respectively, and the action space of the receiver is denoted by $A$. The game proceeds as follows. Nature decides the sender's type $t \in T$, and the result is communicated to the sender only. With knowledge of his type $t$, the sender chooses a signal $s \in S$, incurring a signaling cost depending on both type and signal. Upon observing the sender's signal $s$, the receiver chooses an action $a \in A$. The sender receives a payoff $U_{S}(a, s, t)$ and the receiver receives a payoff $U_{R}(a, s, t)$. Then the game ends.

A (pure) strategy of the sender is a function $\sigma: T \rightarrow S$. A (pure) strategy of the receiver is a function $\gamma: S \rightarrow A$. Since we focus only on pure strategies, we omit the prefix "pure" from now on.

Definition 3.2.1. A strategy pair $(\sigma, \gamma)$ is a separating equilibrium (SE) if the strategy $\sigma$ is one-to-one and, moreover, for all $t \in T$ we have

[1] $U_{S}(\gamma(\sigma(t)), \sigma(t), t) \geq U_{S}(\gamma(s), s, t) \quad$ for all $s \in S$

[2] $U_{R}(\gamma(\sigma(t)), \sigma(t), t) \geq U_{R}(a, \sigma(t), t)$ for all $a \in A$.

If a strategy $\sigma$ of the sender is part of a separating equilibrium, it is called a separating equilibrium strategy (SE strategy).

In the previous chapter, we have restricted our attention on the case that the receiver's payoff is $U_{R}(a, t)$, a mapping of his own action $a$ and the 
Chapter 3. Separating Equilibria in One Dimensional Non-Pure Signaling Games

sender's type $t$. In this chapter, we consider the general case that the receiver's payoff function also depends on the signal $s$ chosen by the sender and so it is now given as $U_{R}(a, s, t)$.

We assume that the sender's payoff $U_{S}: A \times S \times T \rightarrow \mathbb{R}$ is quasilinear in $a$ and so it is given

$$
U_{s}(a, s, t)=a-c(s, t),
$$

where $c: S \times T \rightarrow \mathbb{R}_{+}$is the signaling cost function. Regarding the receiver's payoff, we assume that for every $(s, t) \in S \times T$, there exists an $\alpha(s, t) \in A$ with

$$
U_{R}(\alpha(s, t), s, t)>U_{R}(a, s, t) \quad \text { for all } a \in A \backslash\{\alpha(s, t)\} .
$$

Thus, $\alpha$ is optimal for the receiver when he believes the sender's type is $t$, upon observing the signal $s$. We call $\alpha$ the best action of the receiver.

We assume that the action set $A=\mathbb{R}$, the signal set $S$ is a real interval with a smallest element 0 , the type set $T$ is a real interval with a smallest element $\underline{t}$. We make the following structural assumptions on $c$ and $\alpha$.

Assumptions 1. The cost function $c$ has the following properties

1. $c(s, t)$ is strictly decreasing in $t$ for each $s$.

2. The cost function $c(s, t)$ has decreasing differences. That is, for all $s, s^{\prime} \in$ $S$ and $t, t^{\prime} \in T$ with $s<s^{\prime}$ and $t<t^{\prime}$ it holds that

$$
c\left(s^{\prime}, t\right)-c(s, t)>c\left(s^{\prime}, t^{\prime}\right)-c\left(s, t^{\prime}\right) .
$$

3. $c$ is continuous.

4. For all $t$, the partial derivative $c_{s}(s, t)$ of $c(s, t)$ with respect to $s$ exists and $c_{s}(s, t)$ is continuous on $S \times T$.

Assumptions 2. We make the following assumptions on $\alpha$.

1. $\alpha$ is continuous,

2. $\alpha(s, t)$ is strictly monotone in $t$ for each $s$,

Assumptions 3. The functions $c$ and $\alpha$ together have the following properties 
1. for every $t \in T$, there exists $s^{*}(t) \in S$ such that the mapping $s \mapsto \alpha(s, t)-$ $c(s, t)$ is strictly increasing on $\left\{s \in S \mid s \leq s^{*}(t)\right\}$ and strictly decreasing on $\left\{s \in S \mid s \geq s^{*}(t)\right\}$,

2. the mapping $t \mapsto s^{*}(t)$ is weakly increasing.

3. $s^{*}(\underline{t})=0$.

We can readily interpret the added assumptions above in the context of the job market model by Spence (1973). For instance, Assumption 3.1 says that there is a unique socially optimal level of education for each type of job seeker under complete information. Assumption 3.2 means that the higher type, the higher the socially optimal level of education.

The following results are immediate consequences of our assumptions.

Proposition 3.2.1. Suppose that Assumptions 1.2 and 1.4 hold. The function $c_{s}(s, t)$ is non-increasing in $t$.

Proof. See Proposition 2.5.1.

Proposition 3.2.2. Suppose that Assumptions 1.3, 2.1, 3.1 and 3.2 hold. Then, the mapping $t \mapsto s^{*}(t)$ is continuous.

Proof. Fix $t$. Observe that $\tilde{s}=\lim _{t^{\prime} \uparrow t} s^{*}\left(t^{\prime}\right)$ exists by Assumption 3.2. By definition of $s^{*}$ (Assumption 3.1), for all $t^{\prime} \in T$ we have

$$
\alpha\left(s^{*}\left(t^{\prime}\right), t^{\prime}\right)-c\left(s^{*}\left(t^{\prime}\right), t^{\prime}\right) \geq \alpha\left(s^{*}(t), t^{\prime}\right)-c\left(s^{*}(t), t^{\prime}\right) .
$$

Continuity of $c$ and $\alpha$ (Assumptions 1.3 and 2.1) implies

$$
\alpha(\tilde{s}, t)-c(\tilde{s}, t) \geq \alpha\left(s^{*}(t), t\right)-c\left(s^{*}(t), t\right) .
$$

By Assumption 3.1, we get $\tilde{s}=s^{*}(t)$. By the same line of argument $\lim _{t^{\prime} \downarrow t} s\left(t^{\prime}\right)=$ $s^{*}(t)$. Thus, $s^{*}$ is continuous at $t$.

Lemma 3.2.1. Let $(\sigma, \gamma)$ be an SE. Then,

1. $(\gamma \circ \sigma)(t)=\alpha(\sigma(t), t)$.

2. $\sigma$ is strictly increasing if Assumption 1.2 holds. 
Chapter 3. Separating Equilibria in One Dimensional Non-Pure Signaling Games

Proof. The first claim is an immediate consequence of the definition of $\alpha$ and part [2] of the definition of an SE.

To show the second claim, take $t^{\prime}>t$. It cannot be the case that $\sigma\left(t^{\prime}\right)=$ $\sigma(t)$, because $\sigma$ is one-to-one. Observe, that part [1] of the definition of SE implies that for all $t, t^{\prime}$,

$$
c\left(\sigma\left(t^{\prime}\right), t\right)-c(\sigma(t), t) \geq \gamma\left(\sigma\left(t^{\prime}\right)\right)-\gamma(\sigma(t)) \geq c\left(\sigma\left(t^{\prime}\right), t^{\prime}\right)-c\left(\sigma(t), t^{\prime}\right) .
$$

If $\sigma\left(t^{\prime}\right)<\sigma(t)$, according to Assumption 1.2, we would obtain

$$
c(\sigma(t), t)-c\left(\sigma\left(t^{\prime}\right), t\right)>c\left(\sigma(t), t^{\prime}\right)-c\left(\sigma\left(t^{\prime}\right), t^{\prime}\right),
$$

which is a contradiction to (3.3).

In our effort to analyse SE strategies, the following properties of such strategies will be helpful. A proof is given in Section 3.5.1.

Lemma 3.2.2. Suppose Assumptions 1.3, 2.1-2.2 and 3.1-3.2 hold. Let $(\sigma, \gamma)$ be an SE. Then, the following hold:

1. $\sigma(t) \geq(\leq) s^{*}(t)$ for all $t$ if $\alpha(s, t)$ is strictly increasing (decreasing, resp.) in $t$ for each $s$

2. $\sigma$ is continuous.

\subsubsection{Graph theoretic interpretation of SE}

We provide a graph theoretic interpretation of separating equilibria in this section. The interpretation provides a useful perspective to investigate existence and uniqueness of separating equilibria in Section 3.3. We first recall some notions from graph theory.

\section{Basic terms and facts from graph theory}

A directed graph or digraph is a pair $\mathbf{G}=(V, E)$ where $V$ is an arbitrary set and $E$ is a collection of ordered pairs $(u, v)$ of elements $u, v \in V$ with $u \neq v$. The elements of $V$ are called nodes or vertices, and the elements of $E$ are called arcs. The complete digraph is the digraph $\mathbf{G}=(V, E)$ where $E$ is the set 
of all possible ordered pairs of distinct elements of $V$. An arc length on $\mathbf{G}$ is a function $l: E \rightarrow \mathbb{R}$.

An arc length $l$ on $\mathbf{G}$ is strictly monotone if $l\left(v, v^{\prime}\right)+l\left(v^{\prime}, v\right)>0$ for all $v \neq v^{\prime}$. When the node set $V$ is a set with a partial order $\geq$, the arc length $l$ is decomposition monotone if for any $v, v^{\prime}, v^{\prime \prime} \in V$ with $v^{\prime \prime}>v^{\prime}>v$, we have $l\left(v, v^{\prime \prime}\right)>l\left(v, v^{\prime}\right)+l\left(v^{\prime}, v^{\prime \prime}\right)$ and $l\left(v^{\prime \prime}, v\right)>l\left(v^{\prime \prime}, v^{\prime}\right)+l\left(v^{\prime}, v\right)$, where $>$ is the strict order of $\geq$.

A function $p: V \rightarrow \mathbb{R}$ is called a node potential for arc length $l$ on $\mathbf{G}=$ $(V, E)$ if for all arcs $(u, v) \in E$

$$
p(v)-p(u) \leq l(u, v) .
$$

Adding a constant to a node potential makes it again a node potential. If all node potentials of a graph differ only by a constant we say that the graph has a unique node potential. Generally, this property does not hold.

In $\mathbf{G}=(V, E)$, a path is a vector $\pi=\left(v_{0}, \ldots, v_{k}\right)$ with $\left(v_{i}, v_{i+1}\right) \in E$ for all $i=0, \ldots, k-1$. For two nodes $u, v \in V$, a path from $u$ to $v$ is a path $\left(v_{0}, \ldots, v_{k}\right)$ with $v_{0}=u$ and $v_{k}=v$. For $u, v \in V$, the set of all paths from $u$ to $v$ is denoted by $\Pi(u, v)$.

The length of a path $\pi=\left(v_{0}, \ldots, v_{k}\right)$, denoted for convenience by $l(\pi)$, is defined as

$$
l(\pi)=\sum_{i=0}^{k-1} l\left(v_{i}, v_{i+1}\right)
$$

The distance from $u$ to $v$ with respect to the arc length $l$ is defined by

$$
\operatorname{dist}(u, v)=\inf _{\pi \in \Pi(u, v)} l(\pi) .
$$

The following facts about the relationship between a distance and a node potential on complete graphs are known in graph theory (See, e.g., Heydenreich et al. (2009) in combination with an application in mechanism design).

Fact 3.2.1. A node potential $p$ on a complete digraph $\mathbf{G}=(V, E)$ exists if and only if $\operatorname{dist}(u, v)+\operatorname{dist}(v, u) \geq 0$ for all $u, v \in V$. $^{1}$

\footnotetext{
${ }^{1}$ For simplicity we state this and the following facts for complete digraphs. They hold as well for strongly connected graphs in which every two nodes $u$ and $v$ are connected by a path directed from $u$ to $v$.
} 
Chapter 3. Separating Equilibria in One Dimensional Non-Pure Signaling Games

Fact 3.2.2. A complete digraph $\mathbf{G}=(V, E)$ has a unique potential if and only if $\operatorname{dist}(u, v)+\operatorname{dist}(v, u)=0$ for all $u, v \in V$.

Fact 3.2.3. If a complete digraph $\mathbf{G}=(V, E)$ has a unique node potential, then for any node potential $p, p(v)-p(u)=\operatorname{dist}(u, v)$ for all $u, v \in V$.

\section{Characterization of SE strategy}

We show that separating sender and receiver strategies interplay in equilibrium as arc lengths and node potentials in particular graphs. We need the following definition.

Definition 3.2.2. For the signaling game with type space $T$, the signaling graph $\mathbf{H}$ is the complete digraph with the node set $T$. For a strategy $\sigma: T \rightarrow S$ of the sender, the arc length $l_{\sigma}$ on $\mathbf{H}$ is defined by, for all $t, t^{\prime} \in T$,

$$
l_{\sigma}\left(t, t^{\prime}\right)=c\left(\sigma\left(t^{\prime}\right), t\right)-c(\sigma(t), t) .
$$

Theorem 3.2.1. Suppose that Assumptions 1.1 holds.

Let $\sigma: T \mapsto S$ be a sender's strategy. Then the following are equivalent.

1. $\sigma$ is an SE strategy.

2. $\sigma$, the digraph $\mathbf{H}$ with arc lengths $l_{\sigma}$, and the mapping $t \mapsto \alpha(\sigma(t), t)$ satisfy

(a) $\sigma$ is one-to-one;

(b) The function $t \mapsto \alpha(\sigma(t), t)$ is a node potential for $l_{\sigma}$ on $\mathbf{H}$.

Proof. 1) $\Rightarrow 2$ ): Let $(\sigma, \gamma)$ be an SE. By definition of an SE, $\sigma$ is one-to-one. By property (I) in the definition of an SE and Lemma 3.2.1 we get for any $t, t^{\prime} \in T$

$$
\begin{aligned}
\alpha(\sigma(t), t)-c(\sigma(t), t) & =\gamma(\sigma(t))-c(\sigma(t), t) \\
& \geq \gamma\left(\sigma\left(t^{\prime}\right)\right)-c\left(\sigma\left(t^{\prime}\right), t\right) \\
& =\alpha\left(\sigma\left(t^{\prime}\right), t^{\prime}\right)-c\left(\sigma\left(t^{\prime}\right), t\right)
\end{aligned}
$$

which, given the definition of $l_{\sigma}$, implies

$$
\alpha\left(\sigma\left(t^{\prime}\right), t^{\prime}\right) \leq \alpha(\sigma(t), t)+l_{\sigma}\left(t, t^{\prime}\right) .
$$


2) $\Rightarrow 1$ ): Define $\gamma(s)=\alpha(\sigma(t), t)$ if $s=\sigma(t)$ for some $t \in T$. As $\sigma$ is one-toone, this is well-defined. For $s \notin \sigma(T)$, define $\gamma(s)=\alpha(\sigma(\underline{t}), \underline{t})-c(\sigma(\underline{t}), \underline{t})$. We need to verify properties (I) and (II) from the definition of an SE.

As for (I), let $t \in T$ and $s \in S$.

If $s=\sigma\left(t^{\prime}\right)$ for some $t^{\prime} \in T$, we get

$$
\begin{aligned}
\alpha(\sigma(t), t)-c(\sigma(t), t) & \geq \alpha\left(\sigma\left(t^{\prime}\right), t^{\prime}\right)-c\left(\sigma\left(t^{\prime}\right), t\right) \\
& =\gamma(s)-c(s, t)
\end{aligned}
$$

where the inequality is due to the fact that $\alpha(\sigma(t), t)$ is a node potential with respect to $l_{\sigma}$.

Otherwise, we get

$$
\begin{aligned}
\alpha(\sigma(t), t)-c(\sigma(t), t)) & \geq \alpha(\sigma(\underline{t}), \underline{t})-c(\sigma(\underline{t}), t) \\
& =\gamma(s)+c(\sigma(\underline{t}), \underline{t})-c(\sigma(\underline{t}), t) \\
& \geq \gamma(s) \\
& \geq \gamma(s)-c(s, t) . \quad \quad \text { (because } c(s, t) \geq 0)
\end{aligned}
$$

The first inequality is again due to the fact that $\alpha(\sigma(t), t)$ is a node potential with respect to $l_{\sigma}$. The second inequality comes from Assumption 1.1.

Therefore, property (I) holds.

Property (II) follows from the definition of $\alpha$.

We will show next that our assumptions on $c$ and $\alpha$ allow to establish an explicit formula for node potentials of $\mathbf{H}$, and thereby define a differential equation to be satisfied by $\alpha$ and $\sigma$ in order to constitute a separating equilibrium. The formula is derived by expressing the distance with respect to $l_{\sigma}$, denoted as dist $\sigma$, by an integral, and observing that node potentials in $\mathbf{H}$ are unique.

Lemma 3.2.3. Suppose Assumptions 1.2-1.4 hold. Let $\sigma: T \mapsto S$ be strictly increasing and continuous. Then, the distance with respect to $l_{\sigma}$ on the signaling graph $\mathbf{H}$ is given as

$$
\operatorname{dist}_{\sigma}\left(t, t^{\prime}\right)=\int_{t}^{t^{\prime}} c_{s}(\sigma(x), x) d \sigma(x)
$$

for all $t, t^{\prime} \in T$, where the integral is the Riemann-Stieltjes integral. In particular, $\mathbf{H}$ has a unique node potential. 
Chapter 3. Separating Equilibria in One Dimensional Non-Pure Signaling Games

Proof. The proof is in Section 3.5.2.

Combining Lemma 3.2.3 and Theorem 3.2.1 we get the following corollary.

Corollary 3.2.1. Suppose Assumptions 1.1-1.4, 2.1-2.2 and 3.1-3.2 hold. If $\sigma$ : $T \rightarrow S$ is an SE strategy, then for all $t, t^{\prime}$

$$
\alpha\left(\sigma\left(t^{\prime}\right), t^{\prime}\right)-\alpha(\sigma(t), t)=\int_{t}^{t^{\prime}} c_{s}(\sigma(x), x) d \sigma(x) .
$$

Proof. Observe that by Lemmas 3.2.1 and 3.2.2, $\sigma$ is strictly increasing and continuous. By Lemma 3.2.3, $\mathbf{H}$ has a unique node potential with respect to $l_{\sigma}$. By Fact 3.2.3 and Theorem 3.2.1, we obtain (3.4).

Theorem 3.2.2. Suppose Assumptions 1.1-1.4, 2.1-2.2 and 3.1-3.2 hold.

A strategy $\sigma$ is an SE strategy if and only if

1. $\sigma$ is strictly increasing and continuous;

2. For all $t \in T$,

$$
\alpha(\sigma(t), t)-\alpha(\sigma(\underline{t}), \underline{t})=\int_{\underline{t}}^{t} c_{s}(\sigma(x), x) d \sigma(x) .
$$

Proof. We show both implications separately.

A. Suppose $\sigma$ is an SE strategy. Condition 1 comes from Lemmas 3.2.1 and 3.2.2. In addition, (3.5) holds by (3.4) of Corollary 3.2.1.

B. Conversely, suppose $\sigma$ satisfies conditions $1-2$. We use Theorem 3.2.1.

Condition 1 clearly implies that $\sigma$ is one-to-one. We next verify that $t \mapsto \alpha(\sigma(t), t)$ is a node potential for $l_{\sigma}$ on $H$. Observe that for all $t, t^{\prime} \in T$

$$
\begin{array}{rlr}
\alpha\left(\sigma\left(t^{\prime}\right), t^{\prime}\right)-\alpha(\sigma(t), t) & =\int_{t}^{t^{\prime}} c_{s}(\sigma(x), x) d \sigma(x) & \text { (by condition 2) } \\
& =\operatorname{dist}_{\sigma}\left(t, t^{\prime}\right) & \text { (by Lemma 3.2.3) } \\
& \leq l_{\sigma}\left(t, t^{\prime}\right) & \text { (by definition of distance) }
\end{array}
$$

Thus, $t \mapsto \alpha(\sigma(t), t)$ is a node potential for $l_{\sigma}$ on $\mathbf{H}$ as desired. The desired result follows. 


\subsection{Existence and Uniqueness of SE strategies}

Theorem 3.2.2 says it is essential to solve integral equation (3.5) for $\sigma$ in order to find an SE strategy. In this section, we examine the existence and uniqueness of solutions of (3.5) under the environment with $S=\mathbb{R}_{+}$and $T=[\underline{t},+\infty)$. Throughout this section, we suppose that Assumptions 1.11.4, 2.1-2.2 and 3.1-3.2 hold. We need to extend them as follows:

Assumptions 1. We add the following structural assumption on $c$.

5. $c(s, t)$ is strictly increasing in $s$ for each $t$.

Assumptions 2. We add the following structural assumptions on $\alpha$.

3. For each $(s, a) \in S \times A$, there exists $t \in T$ such that $\alpha(s, t)=a$.

4. For each $s, \lim _{t \rightarrow+\infty} \alpha(s, t)=+\infty$.

To state results, it is convenient to define a new mapping. Observe that for all $(s, a) \in S \times A=\mathbb{R}_{+} \times \mathbb{R}$, there uniquely exists $t \in T$ such that $\alpha(s, t)=a$ due to Assumptions 2.2 and 2.3. We denote such $t$ by $\beta(s, a)$. That is, $\alpha(s, \beta(s, a))=a$ for $(s, a) \in S \times A$. In the pure signaling case, it is simply $\beta(s, a)=\alpha^{-1}(a)$. We define $H$ on $\mathbb{R}_{+} \times \mathbb{R}$ by $H(s, a)=c_{s}(s, \beta(s, a))$.

Lemma 3.3.1. The function $H$ is continuous on $\mathbb{R}_{+} \times \mathbb{R}$ and the function $a \mapsto$ $H(s, a)$ is non-increasing for all $s$.

Proof. To see continuity, it suffices to show that $\beta$ is continuous since $c_{s}$ is assumed to be continuous (Assumption 1.4). Let $F: S \times A \times T \rightarrow \mathbb{R}$ be a continuous mapping defined by $F(t, s, a)=\alpha(s, t)-a$. Take a sequence $\left\{\left(s_{n}, a_{n}\right)\right\}$ in $\mathbb{R}_{+} \times \mathbb{R}$ converging to $(s, a)$. There corresponds the unique sequence $\left\{t_{n}\right\} \subset T$ such that $\alpha\left(s_{n}, t_{n}\right)=a$, as claimed above. Let $t$ be a point such that $F(t, s, a)=\alpha(s, t)-a=0$.

If $\left\{t_{n}\right\} \rightarrow \infty$, there exist an open neighborhood $U$ of $t$ and an integer $N$ such that $t_{n} \notin \mathrm{cl} U$ for any $n>N$, where $\mathrm{cl} U$ is the closure of $U$.

Since $F(t, s, a)=0$, there exists sufficiently small neighborhood $V$ of $(s, a)$ such that for any $(\tilde{s}, \tilde{a}) \in V, F(U, \tilde{s}, \tilde{a})$ contains 0 in its interior. This implies that for any $(\tilde{s}, \tilde{a}) \in V$, there exists unique $\tilde{t} \in U$ such that $F(\tilde{t}, \tilde{s}, \tilde{a})=$ 0 . Since $\left(s_{n}, a_{n}\right) \rightarrow(s, a)$, there exists an integer $M$ such that for any $n>M$, 
Chapter 3. Separating Equilibria in One Dimensional Non-Pure Signaling Games

$\left(s_{n}, a_{n}\right) \in V$. Then, for any $n>M, t_{n} \in U$, and this leads to a contradiction. Therefore, $\left\{t_{n}\right\}$ is bounded and has convergent subsequence $\left\{t_{n_{k}}\right\} \rightarrow t^{\prime}$. By the continuity of $F$,

$$
\lim _{k \rightarrow \infty} F\left(t_{n_{k}}, s_{n_{k}}, a_{n_{k}}\right)=F\left(\lim _{k \rightarrow \infty} x, \lim _{k \rightarrow \infty} s_{n_{k}}, \lim _{k \rightarrow \infty} a_{n_{k}}\right)=F\left(t^{\prime}, s, a\right)=0 .
$$

Then, we have $t^{\prime}=t$ by the uniqueness. This implies that $\left\{t_{n}\right\}$ itself converges to $t$ and thus $\beta$ is continuous.

Observe that

$$
\begin{aligned}
\alpha(s, \beta(s, a)) & =a \\
& =\lim _{n \rightarrow \infty} a_{n} \\
& =\lim _{n \rightarrow \infty} \alpha\left(s_{n}, \beta\left(s_{n}, a_{n}\right)\right) \\
& =\alpha\left(s, \lim _{n \rightarrow+\infty} \beta\left(s_{n}, a_{n}\right)\right) .
\end{aligned}
$$

The first and third equality hold by definition of $\beta$ and the last one due to the continuity of $\alpha$ (Assumption 2.1).

Thus, by Assumption $2.2 \beta(s, a)=\lim _{n \rightarrow+\infty} \beta\left(s_{n}, a_{n}\right)$, as desired.

We show the second claim. Due to Proposition 3.2.1, it suffices to show that for each $s$ the function $a \mapsto \beta(s, a)$ is strictly increasing. Fix $s$.

Let $a^{\prime}>a$. Then, it holds that

$$
\alpha\left(s, \beta\left(s, a^{\prime}\right)\right)-\alpha(s, \beta(s, a))=a^{\prime}-a>0 .
$$

Since $\alpha$ is strictly increasing in the second argument by Assumptions 2.2 and 2.4, the inequality $\beta\left(s, a^{\prime}\right)>\beta(s, a)$ follows as desired.

Choose $\underline{s} \in S$ and consider the initial value problem on $[\underline{s}, \infty)$

$$
f^{\prime}(s)=H(s, f(s)) \text { with } f(\underline{s})=\alpha(\underline{s}, \underline{t}) .
$$

We denote by $f_{\underline{s}}$ a solution of the initial value problem (3.6) on $[\underline{s}, \infty)$, if it exists. When $f_{\underline{s}}$ exists, we define the mapping $\tau_{\underline{s}}:[\underline{s}, \infty) \rightarrow \mathbb{R}$ as

$$
\tau_{\underline{s}}(s)=\beta\left(s, f_{\underline{s}}(s)\right) .
$$

Theorem 3.3.1. Let $S=\mathbb{R}_{+}, T=[\underline{t},+\infty)$. Suppose Assumptions 1.1-1.5, 2.1-2.4 and 3.1-3.2 are respected. Then, the following hold. 
1. Let $\underline{s} \in S$ and suppose $f_{\underline{s}}$ exists. If $\tau_{\underline{s}}$ is strictly increasing and $\lim _{s \rightarrow \infty} \tau_{\underline{s}}(s)=$ $\infty$, then $\sigma=\tau_{\underline{s}}^{-1}$ is an SE strategy with $\sigma(\underline{t})=\underline{s}$.

2. Suppose $\sigma$ is an SE strategy. Then,

(a) the mapping $s \mapsto \alpha\left(s, \sigma^{-1}(s)\right)$ is a solution to the initial value problem $(3.6)[\underline{s}, \infty)$ where $\underline{s}=\sigma(\underline{t})$,

(b) $\tau_{\underline{s}}$ is strictly increasing and $\lim _{s \rightarrow \infty} \tau_{\underline{s}}(s)=\infty$,

(c) $\sigma=\tau_{\underline{s}}^{-1}$.

Proof. 1. Fix $\underline{s} \in S$. Suppose $f_{\underline{s}}$ exists and $\tau_{\underline{s}}$ is strictly increasing and goes to infinity. Since by construction of $\tau_{\underline{s}}$ and $\beta$

$$
\tau_{\underline{s}}(\underline{s})=\beta\left(\underline{s}, f_{\underline{s}}(\underline{s})\right)=\beta(\underline{s}, \alpha(\underline{s}, \underline{t}))=\underline{t}
$$

the range of $\tau_{\underline{s}}$ is $[\underline{t},+\infty)$. Hence, its inverse, denoted as $\sigma$, exists on $T \equiv$ $[\underline{t},+\infty)$. We show that $\sigma$ is an SE strategy using Theorem 3.2.2.

Obviously, $\sigma$ is strictly increasing and continuous because so is its inverse $\tau_{\underline{s}}$. In addition, for $s, s^{\prime} \in \sigma(T)$

$$
\begin{array}{rlr}
\int_{s^{\prime}}^{s} c_{s}\left(y, \sigma^{-1}(y)\right) d y & =\int_{s^{\prime}}^{s} c_{s}\left(y, \tau_{\underline{s}}(y)\right) d y \\
& =\int_{s^{\prime}}^{s} c_{s}\left(y, \beta\left(y, f_{\underline{s}}(y)\right)\right) d y & \text { (by definition of } \left.\tau_{\underline{s}}\right) \\
& =f_{\underline{s}}(s)-f_{\underline{s}}\left(s^{\prime}\right) \\
& =\alpha\left(s, \sigma^{-1}(s)\right)-\alpha\left(s^{\prime}, \sigma^{-1}\left(s^{\prime}\right)\right) . & (\text { by }(3.6)) \\
& & \text { (because } \left.\sigma^{-1}(s)=\tau(s)=\beta(s, \bar{f}(s))\right)
\end{array}
$$

By the change of variable $y=\sigma(x)$, we obtain

$$
\alpha(\sigma(t), t)-\alpha\left(\sigma\left(t^{\prime}\right), t^{\prime}\right)=\int_{\underline{t}}^{t} c_{s}(\sigma(x), x) d \sigma(x),
$$

which is equation (3.5) in Theorem 3.2.2.

Therefore, we conclude that $\sigma$ is an SE strategy.

2. Let $\sigma$ be an SE strategy and $\sigma(\underline{t})=\underline{s}$. By Theorem 3.2.2, $\sigma$ is continuous and strictly increasing. Hence, either $\sigma(T)=[\underline{s}, \infty)$, or there is $\bar{s}<\infty$ with $\sigma(T)=[\underline{s}, \bar{s})$, and $\sigma^{-1}$ exists on $\sigma(T)$. Define

$$
f(s)=\alpha\left(s, \sigma^{-1}(s)\right) \quad \text { for } s \in \sigma(T) .
$$


Chapter 3. Separating Equilibria in One Dimensional Non-Pure Signaling Games

Note that $f(\underline{s})=\alpha\left(\underline{s}, \sigma^{-1}(\underline{s})\right)=\alpha(\underline{s}, \underline{t})$. In addition, for $s \in \sigma(T)$

$$
\begin{aligned}
f(s) & =\alpha\left(s, \sigma^{-1}(s)\right) \\
& =\int_{\underline{s}}^{s} c_{s}\left(y, \sigma^{-1}(y)\right) d y+\alpha\left(\underline{s}, \sigma^{-1}(\underline{s})\right) \\
& =\int_{\underline{s}}^{s} c_{s}\left(y, \beta(y, f(y)) d y+\alpha\left(\underline{s}, \sigma^{-1}(\underline{s})\right)\right. \\
& =\int_{\underline{s}}^{s} H(y, f(y)) d y+\alpha\left(\underline{s}, \sigma^{-1}(\underline{s})\right),
\end{aligned}
$$

where the second equality follows by applying the change of variable to (3.5) in Theorem 3.2.2 and the third by the definition of $\beta$.

Since the function $y \mapsto H(y, f(y))$ is continuous by Lemma 3.3.1, the Fundamental Theorem of Calculus states that $f$ is differentiable, and that for all $s \in \sigma(T)$

$$
f^{\prime}(s)=H(s, f(s)) \text { with } f(\underline{s})=\alpha(\underline{s}, \underline{t}) .
$$

Then, we see that $f$ is a partial solution on $\sigma(T)$ to the initial value problem (3.6).

We now show that $\sigma(T)=[\underline{s}, \infty)$. Suppose not, i.e., $\sigma(T)=[\underline{s}, \bar{s})$. To derive a contradiction, observe that for $s \in \sigma(T)$

$$
\begin{aligned}
\alpha\left(s, \sigma^{-1}(s)\right) & =\int_{\underline{s}}^{s} c_{s}\left(y, \sigma^{-1}(y)\right) d y+\alpha\left(\underline{s}, \sigma^{-1}(\underline{s})\right) \\
& \leq \int_{\underline{s}}^{s} c_{s}\left(y, \sigma^{-1}(\underline{s})\right) d y+\alpha\left(\underline{s}, \sigma^{-1}(\underline{s})\right) \quad(\text { by Proposition 3.2.1) } \\
& =c\left(s, \sigma^{-1}(\underline{s})\right)-c\left(\underline{s}, \sigma^{-1}(\underline{s})\right)+\alpha\left(\underline{s}, \sigma^{-1}(\underline{s})\right)
\end{aligned}
$$

Letting $s \rightarrow \bar{s}$ yields

$$
\alpha\left(\bar{s}, \lim _{s \rightarrow \bar{s}} \sigma^{-1}(s)\right) \leq c\left(\bar{s}, \sigma^{-1}(\underline{s})\right)-c\left(\underline{s}, \sigma^{-1}(\underline{s})\right)+\alpha\left(\underline{s}, \sigma^{-1}(\underline{s})\right)<\infty .
$$

However, since $\lim _{s \rightarrow \bar{s}} \sigma^{-1}(s)=\infty$, by Assumption 2.4 it holds

$$
\alpha\left(\bar{s}, \lim _{s \rightarrow \bar{s}} \sigma^{-1}(s)\right)=\infty .
$$

This is a contradiction. Hence, $\sigma(T)=[\underline{s}, \infty)$, and $f$ is indeed a solution of the initial value problem (3.6). Claim (a) follows. 
Moreover, for each $s \in[\underline{s}, \infty)$ we have $f(s)=\alpha\left(s, \sigma^{-1}(s)\right)$ and so $\sigma^{-1}(s)=$ $\beta(s, f(s))=\tau_{\underline{s}}(s)$. Claim (c) follows.

Finally, noting that $\sigma$ is strictly increasing and defined on $T=[\underline{t}, \infty)$, we conclude claim (b) that $\tau_{\underline{s}}$ is strictly increasing and goes to infinity.

According to the theorem above, it should be a starting step to solve the initial value problem (3.6) to determine an SE strategy. Then, it is natural that the question will arise about existence and uniqueness of solutions of the initial value problem.

Theorem 3.3.2. Let $S=\mathbb{R}_{+}, T=[\underline{t},+\infty)$. Suppose Assumptions 1.1-1.5, 2.1-2.4 and 3.1-3.2 hold.

1. For each $\underline{s} \geq 0$, the initial value problem (3.6) has at most one solution.

2. If $c_{s}(s, t)$ is bounded on $S \times T$, then for each $\underline{s} \geq 0$ the initial value problem (3.6) has a unique solution $f_{\underline{s}}$.

3. If $\sigma_{1}$ and $\sigma_{2}$ are SE strategies with $\sigma_{1}(\underline{t})=\sigma_{2}(\underline{t})=\underline{s}$, then $\sigma_{1}(s)=\sigma_{2}(s)$ for all $s \geq \underline{s}$.

Proof. 1. $\quad H$ is continuous on $\mathbb{R}_{+} \times \mathbb{R}$ and non-increasing in the second argument by Lemma 3.3.1. The desired result immediately follows from Theorem 3.5.2.

2. $\quad H$ is continuous on $\mathbb{R}_{+} \times \mathbb{R}$ by Lemma 3.3.1 and bounded by assumption. Hence, the initial value problem (3.6) has a solution by Theorem 3.5.1. Uniqueness comes from the first claim.

3. If both $\sigma_{1}$ and $\sigma_{2}$ are SE strategies, two mappings $s \mapsto \alpha\left(s, \sigma_{1}^{-1}(s)\right)$ and $s \mapsto \alpha\left(s, \sigma_{2}^{-1}(s)\right)$ are solutions of the same initial value problem (3.6) by the second claim of Theorem 3.3.1. But, by the first claim above, it is the case that $\alpha\left(s, \sigma_{1}^{-1}(s)\right)=\alpha\left(s, \sigma_{2}^{-1}(s)\right)$ for all $s \geq \underline{s}$. Because $\alpha$ is assumed to be strictly monotone in the second argument (Assumption 2.2), $\sigma_{1}^{-1}=\sigma_{2}^{-1}$ in $[\underline{s}, \infty)$. The desired result follows.

\subsection{Applications}

We apply our results to two economic settings. We revisit Example 3.1.1, a signaling game with a loss averse sender. This game does not fall within 
Chapter 3. Separating Equilibria in One Dimensional Non-Pure Signaling Games

the scope of either Mailath (1987) or Mailath and von Thadden (2013) due to the presence of a kink. The other is a signaling game arising in a security design problem in which an issuer of bonds backed by an asset has private information about the value of the asset. Mailath (1987) cannot be applied to this example while its recent extension by Mailath and von Thadden (2013) can be.

For the sake of discussion and comparison with Mailath (1987) and Mailath and von Thadden (2013), it is convenient to introduce the reduced form payoff to a sender, the function $V: T \times T \times S \rightarrow \mathbb{R}$ defined as

$$
V\left(t, t^{\prime}, s\right)=U_{S}\left(\alpha\left(s, t^{\prime}\right), s, t\right) .
$$

This is interpreted as the payoff to the type $t$ sender when he chooses signal $s$ and a receiver believes a sender to be of type $t^{\prime}$.

\subsubsection{Signaling with a loss averse sender}

We reconsider the signaling game $\mathscr{G}$ with a loss averse sender in Example 3.1.1. Recall that the sender's payoff $\hat{U}_{S}: A \times S \times T \rightarrow \mathbb{R}$ is

$$
\hat{U}_{S}(a, s, t)=\left\{\begin{array}{cl}
a-c(s, t) & \text { if } a \leq r \\
a+k(a-r)-c(s, t) & \text { otherwise. }
\end{array}\right.
$$

where $k>0$ is a constant, $\lambda$ is the reference point and $c$ is the signaling cost function. We will find an SE strategy of $\mathscr{G}$ using our results. The following lemma is useful.

Lemma 3.4.1. Suppose that the cost function c satisfies Assumption 1.1. Let $\sigma$ be a mapping from $S$ to $T$. Then, $\sigma$ is an $S E$ strategy of $C$ if and only if it is an SE strategy of the signaling game with the signaling cost function $c$ and the best action of the sender $\tilde{\alpha}$ defined as

$$
\tilde{\alpha}(s, t)=\left\{\begin{array}{cl}
\alpha(s, t) & \text { if } \alpha(s, t) \leq r \\
\alpha(s, t)+k(\alpha(s, t)-\lambda) & \text { otherwise. }
\end{array}\right.
$$

Proof. By Theorem 3.2.1, it suffices to show that $\sigma$ is an SE strategy of $\mathscr{G}$ if and only if (1) it is one-to-one and (2) the function $t \mapsto \tilde{\alpha}(\sigma(t), t)$ is a node potential for $l_{\sigma}$ on $H$. 
Notice that if $(\sigma, \gamma)$ is an SE of $\mathscr{G}$, then $(\gamma \circ \sigma)(t)=\alpha(\sigma(t), t)$ due to the definition of $\alpha$ and part [2] of Definition 3.2.1.

$(\Longrightarrow)$ Suppose $(\sigma, \gamma)$ is an SE. Then, by definition of an SE, $\sigma$ is oneto-one. In addition, for any $t, t^{\prime} \in T$

$$
\begin{aligned}
\tilde{\alpha}(\sigma(t), t)-c(\sigma(t), t) & =\hat{U}_{s}(\alpha(\sigma(t), t), \sigma(t), t) \\
& =\hat{U}_{s}(\gamma(\sigma(t)), \sigma(t), t) \\
& \geq \hat{U}_{s}\left(\gamma\left(\sigma\left(t^{\prime}\right)\right), \sigma\left(t^{\prime}\right), t\right) \\
& =\hat{U}_{s}\left(\alpha\left(\sigma\left(t^{\prime}\right), t^{\prime}\right), \sigma\left(t^{\prime}\right), t\right) \\
& =\tilde{\alpha}\left(\sigma\left(t^{\prime}\right), t^{\prime}\right)-c\left(\sigma\left(t^{\prime}\right), t\right)
\end{aligned}
$$

(by definition of an SE)

which, given the definition of $l_{\sigma}$, implies

$$
\tilde{\alpha}\left(\sigma\left(t^{\prime}\right), t^{\prime}\right) \leq \tilde{\alpha}(\sigma(t), t)+l_{\sigma}\left(t, t^{\prime}\right) .
$$

$(\Leftarrow=) \quad$ Suppose $\sigma$ satisfies conditions (1) and (2). Define $\gamma(s)=\alpha(\sigma(t), t)$ if $s=\sigma(t)$ for some $t \in T$. As $\sigma$ is one-to-one, this is well-defined. For $s \notin$ $\sigma(T)$, define $\gamma(s)=\min \{r, \tilde{\alpha}(\sigma(\underline{t}), \underline{t})-c(\sigma(\underline{t}), \underline{t})\}$. We need to verify properties (I) and (II) in Definition 3.2.1.

As for (I), let $t \in T$ and $s \in S$.

If $s=\sigma\left(t^{\prime}\right)$ for some $t^{\prime} \in T$, we get

$$
\begin{aligned}
\hat{U}_{s}(\gamma(\sigma(t)), \sigma(t), t) & =\hat{U}_{s}(\alpha(\sigma(t), t), \sigma(t), t)=\tilde{\alpha}(\sigma(t), t)-c(\sigma(t), t) \\
& \geq \tilde{\alpha}\left(\sigma\left(t^{\prime}\right), t^{\prime}\right)-c\left(\sigma\left(t^{\prime}\right), t\right) \quad(\text { by condition }(2)) \\
& =\hat{U}_{s}\left(\alpha\left(\sigma\left(t^{\prime}\right), t^{\prime}\right), \sigma\left(t^{\prime}\right), t\right)=\hat{U}_{s}(\gamma(s), s, t) .
\end{aligned}
$$

Otherwise, we get

$$
\begin{array}{rlr}
\hat{U}_{s}(\gamma(\sigma(t)), \sigma(t), t) & =\hat{U}_{s}(\alpha(\sigma(t), t), \sigma(t), t) \\
& =\tilde{\alpha}(\sigma(t), t)-c(\sigma(t), t) \\
& \geq \tilde{\alpha}(\sigma(\underline{t}), \underline{t})-c(\sigma(\underline{t}), t) & \\
& \geq \gamma(s)+c(\sigma(\underline{t}), \underline{t})-c(\sigma(\underline{t}), t) & \text { (by condition }(2)) \\
& \geq \gamma(s) & \text { (by contruction of } \gamma(s)) \\
& \geq \gamma(s)-c(s, t) . & \text { (by Assumption 1.1) } \\
& =\hat{U}_{s}(\gamma(s), s, t) & (\text { for } \gamma(s) \leq r \text { by contruction of } \gamma(s))
\end{array}
$$


Chapter 3. Separating Equilibria in One Dimensional Non-Pure Signaling Games

Therefore, property (I) holds.

Property (II) follows from the definition of $\alpha$.

To illustrate how to find an SE strategy of $\mathscr{G}$, we consider the example that $S=\mathbb{R}_{+}, T=[\underline{t}, \infty)=[1, \infty)$ and the cost function $c(s, t)=(s+1)^{2} / t$ and the best action of the receiver $\alpha(s, t)=(s+1) t$. Assume further that the reference point of type $t$ sender is given as $r=(\lambda+1) t$ with $\lambda>0$.

Due to Lemma 3.4.1, an SE strategy of $\mathscr{G}$ is an SE strategy of the signaling game with $c$ and $\tilde{\alpha}$, where

$$
\tilde{\alpha}(s, t)=\left\{\begin{array}{cl}
(s+1) t & \text { if } s \leq \lambda \\
(s+1) t+k(s-\lambda) t & \text { otherwise. }
\end{array}\right.
$$

We first check that Assumptions 1.1-1.5, Assumptions 2.1-2.4 and Assumptions 3.1-3.2 are respected in the current setup.

- Clearly, $c(s, t)=(s+1)^{2} / t$ is strictly decreasing (increasing, resp.) in $t$ $(s$, resp.) for each $s(t$, resp.). It is also continuous and has decreasing differences. The partial derivative $c_{s}(s, t)=2(s+1) / t$ is continuous on $S \times T$. Hence, Assumptions 1.1-1.5 hold.

- The function $(s, t) \mapsto \alpha(s, t)=(s+1) t$ is continuous and it is strictly increasing in $t$ and goes to infinity for each $s$. In addition, for $(s, a) \epsilon$ $S \times A=\mathbb{R}_{+} \times \mathbb{R}_{+}$, there exists $t$ such that $(s+1) t=a$. Thus, Assumptions 2.1-2.4 are satisfied.

- For each $t$, the function $s \mapsto \alpha(s, t)-c(s, t)=(s+1) t-(s+1)^{2} / t$ is singlepeaked at $s=\max \left(0, t^{2} / 2-1\right)$. Hence, Assumptions 3.1-3.2 hold.

Thus, we can use Theorem 3.3.1.

We have

$$
\beta(s, a)= \begin{cases}\frac{a}{s+1} & \text { if } s \leq \lambda \\ \frac{a}{s+1+k(s-\lambda)} & \text { otherwise }\end{cases}
$$

and so

$$
H(s, a)=c_{s}(s, \beta(s, a))= \begin{cases}\frac{2(s+1)^{2}}{a} & \text { if } s \leq \lambda \\ \frac{2(s+1)(s+1+k(s-\lambda))}{a} & \text { otherwise. }\end{cases}
$$


Choose $\underline{s} \in S$. The initial value problem (3.6) becomes

$$
f^{\prime}(s)=H(s, f(s)) \text { with } f(\underline{s})=\alpha(\underline{s}, \underline{t})=(\underline{s}+1) \underline{t}=\underline{s}+1
$$

and its solution is

$$
f_{\underline{s}}(s)= \begin{cases}\sqrt{h(s, 0)-h(\underline{s}, 0)+\underline{s}+1} & \text { if } 0 \leq s \leq \lambda \\ \sqrt{h(s, k)-h(\underline{s}, k)+\underline{s}+1} & \text { if } s \geq \lambda\end{cases}
$$

where $h(s, k)=\frac{4}{3}(s+1)^{3}+\frac{2 k}{3} s^{3}+2 k(1-\lambda) s^{2}-4 k \lambda s$.

We obtain

$$
\tau_{\underline{s}}(s)=\beta\left(s, f_{\underline{s}}(s)\right)= \begin{cases}\frac{\sqrt{h(s, 0)-h(\underline{s}, 0)+\underline{s}+1}}{s+1} & \text { if } 0 \leq s \leq \lambda \\ \frac{\sqrt{h(s, k)-h(\underline{s}, k)+\underline{s}+1}}{s+1+k(s-\lambda)} & \text { if } s \geq \lambda\end{cases}
$$

Since $\tau_{\underline{s}}$ is strictly increasing and $\tau_{\underline{s}}(s) \rightarrow+\infty$ as $s \rightarrow+\infty$, by the first claim of Theorem 3.3.1 we conclude that $\bar{\sigma}=\tau^{-1}$ is an SE strategy with $\sigma(\underline{t})=\underline{s}$. In fact, by the third claim of Theorem 3.3.2 it is the unique SE strategy among the ones having the properties $\sigma(\underline{t})=\underline{s}$.

\subsubsection{Security design}

We consider the security design model by DeMarzo and Duffie (1999). In their model, an issuer of bonds backed by assets has private information about the value of the assets. As such, information asymmetry issue naturally arises from the process of selling the bonds to outside investors.

In the model, risk neutral participants consist of an issuer (the sender) and a set of outside investors (the receiver). The issuer owns assets that generate future cash flows given by a random variable $X$ and is in need of cash now. To raise cash, the issuer designs a security backed by these assets and sells some portion of these assets to outside investors. But, after designing the security and before the sale, the issuer gets private information $t$ about the distribution of future cash flows $X$. Precisely, the timeline is as follows:

- In stage 1 , the issuer designs a security with promised payment $F \leq$ $X$; 
Chapter 3. Separating Equilibria in One Dimensional Non-Pure Signaling Games

- In stage 2, the issuer receives private information $Z$ about $X$;

- In stage 3, the issuer sells to outside investors fraction $s \in[0,1]$ of the security at price $a$. The price of the security determined in a Walrasian market setting.

- In stage 4 , the cash flow $X$ is realized and the issuer pays $F \cdot s$ to outside investors. The issuer's total ex-post payoff sums the values of the residual cash flow, unsold fraction of the security and the cash flow raised from the sale of the security: $\delta(X-F)+\delta(1-s) F+a$ where $\delta \in(0,1)$ is the discounting rate.

Notice that a lemon problem arises in stage 3. If private information received in the second stage indicates that $X$ is likely to realize low cash flow, because $\delta<1$, it will be optimal for the issuer to sell all of the security $(s=1)$ at a given price. But, outside investors interpret a high quantity sold as bad news about expected realization of cash flows and so offer low price. Thus, the choice $s$ of fraction plays the role of signal for $Z$.

Formally, the signaling game which the issuer and investors play through stages 2 and 3 is as follows. The issuer is informed of $Z$ and his private information is summarized into $t \equiv \mathbb{E}(F \mid Z) \in \mathbb{R}$. Then, he chooses a quantity $s$ to put up for sale to uninformed investors, who pay $a$ in exchange. The issuer's expected payoff $U: A \times S \times T \rightarrow \mathbb{R}$ is written as

$$
\begin{aligned}
U(a, s, t) & =\delta \mathbb{E}(X-F \mid Z)+\delta(1-s) \mathbb{E}(F \mid Z)+a \\
& =a-\delta s \cdot t+\delta \mathbb{E}(X \mid Z)
\end{aligned}
$$

Here, notice that the term $\delta \mathbb{E}(X \mid Z)$ is a constant and so we can safely ignore it in the analysis.

Investors, who bid for the securities, are competitive and must make zero expected payoffs for each type $t$. Hence if they believe the issuer's type to be of type $t$, the equilibrium price of the security will be simply $\mathbb{E}(F \mid Z) \equiv t$. So, under complete information investors will pay to the issuer the amount $\alpha(s, t)=s \cdot t$.

In sum, the signaling game under consideration is such that $T=\left[t_{0}, t_{1}\right]$ or $\left[t_{0}, \infty\right), A=\mathbb{R}_{+}, S=[0,1]$ and the best action of the receiver and the cost 
function of the sender are, respectively,

$$
\alpha(s, t)=s \cdot t \text { and } c(s, t)=\delta s \cdot t .
$$

DeMarzo and Duffie (1999) consider the bounded type set $T=\left[t_{0}, t_{1}\right]$ with $t_{0}>0$ and show the following proposition.

Proposition 3.4.1 (Proposition 2 in DeMarzo and Duffie (1999)). The function $\sigma: T \rightarrow S$ defined as $\sigma(t)=\left(\frac{t_{0}}{t}\right)^{1 /(1-\delta)}$ is the unique SE strategy.

Their proof is based on the results of Mailath (1987). But, the reduced form payoff to the issuer is

$$
V\left(t, t^{\prime}, s\right) \equiv U\left(\alpha\left(s, t^{\prime}\right), s, t\right)=s \cdot t^{\prime}-\delta s \cdot t
$$

and so it is affine in $s$. Such affinity violates one of the assumptions under which the results of Mailath (1987) are valid (See Section 2.6). The recent paper by Mailath and von Thadden (2013) presents the extension theorem which takes this affinity into account as well. Here, we provide the correct proof using our results.

Proof of Proposition 3.4.1. To fit this game to our setting, we take the change of variable $\tilde{t}=1 / t$. Then, we have the type set $\tilde{T}=\left[\frac{1}{t_{1}}, \frac{1}{t_{0}}\right]$ and

$$
\alpha(s, \tilde{t})=\frac{s}{\tilde{t}} \text { and } c(s, \tilde{t})=\delta \frac{s}{\tilde{t}} .
$$

We verify that Assumptions 1.1-1.4, Assumptions 2.1-2.2 and Assumptions 3.1-3.2 are respected in the current setup.

- Clearly, $c(s, \tilde{t})=\delta s / \tilde{t}$ is strictly decreasing in $\tilde{t}$ for each $s$. It is also continuous and has decreasing differences. The partial derivative $c_{s}(s, \tilde{t})=1 / \tilde{t}$ is continuous on $S \times \tilde{T}$. Hence, Assumptions 1.1-1.4 hold.

- The function $(s, \tilde{t}) \mapsto \alpha(s, \tilde{t})=s / \tilde{t}$ is continuous and it is strictly decreasing in $\tilde{t}$ for each $s$. Thus, Assumptions 2.1-2.2 are satisfied.

- For each $\tilde{t}$, the function $s \mapsto \alpha(s, \tilde{t})-c(s, \tilde{t})=s / \tilde{t}-\delta s / \tilde{t}$ is single-peaked at $s=1$. Hence, Assumptions 3.1-3.2 hold. 
Chapter 3. Separating Equilibria in One Dimensional Non-Pure Signaling Games

Thus, we can use Theorem 3.2.2.

Suppose $\sigma$ is an SE strategy. By Theorem 3.2.2, it is continuous and strictly increasing. In addition, for all $\tilde{t}, \tilde{t}^{\prime} \in \tilde{T}$

$$
\begin{aligned}
\frac{\sigma(\tilde{t})}{\tilde{t}}-\frac{\sigma\left(\tilde{t}^{\prime}\right)}{\tilde{t}^{\prime}} & =\int_{\tilde{t}^{\prime}}^{\tilde{x}} \frac{\delta}{x} d \sigma(x) \\
& =\delta\left(\frac{\sigma(\tilde{t})}{\tilde{t}}-\frac{\sigma\left(\tilde{t}^{\prime}\right)}{\tilde{t}^{\prime}}\right)+\int_{\tilde{t}^{\prime}}^{\tilde{t}} \frac{\delta \sigma(x)}{x^{2}} d x
\end{aligned}
$$

Here, the first equality is due to (3.6) in Theorem 3.2.2 and the second equality is due to integration-by-parts.

As such, we have

$$
\frac{\sigma(\tilde{t})}{\tilde{t}}-\frac{\sigma\left(\tilde{t}^{\prime}\right)}{\tilde{t}^{\prime}}=\frac{\delta}{(1-\delta)} \int_{\tilde{t}^{\prime}}^{\tilde{t}} \frac{\sigma(x)}{x^{2}} d x
$$

Since the integrand $\frac{\sigma(x)}{x^{2}}$ is continuous, the term in the right hand side is differentiable with respect to $\tilde{t}$ by the Fundamental Theorem of Calculus, which in turn implies that $\sigma$ on the left hand side is also differentiable.

Differentiating both sides of (3.9) with respect to $\tilde{t}$ yields the differential equation

$$
\frac{\tilde{t} \sigma^{\prime}(\tilde{t})-\sigma(\tilde{t})}{\tilde{t}^{2}}=\frac{\delta}{(1-\delta)} \frac{\sigma(\tilde{t})}{\tilde{t}^{2}}
$$

Solving this differential equation gives $\sigma(\tilde{t})=C \cdot \tilde{t}^{1 /(1-\delta)}$ for some constant C.

As DeMarzo and Duffie (1999) argue, the lowest type $t_{0}$ indicates that the asset at hands will realize the lowest cash flow, and so the issuer of type $t_{0}$ will sell all of the security $(s=1)$. This means $\sigma\left(1 / t_{0}\right)=1$ and thus

$$
\sigma(\tilde{t})=\left(t_{0} \cdot \tilde{t}\right)^{1 /(1-\delta)}
$$

By Theorem 3.2.2, we conclude that $\sigma(\tilde{t})=\left(t_{0} \cdot \tilde{t}\right)^{1 /(1-\delta)}$ is the unique SE strategy. 


\subsection{Appendix}

\subsubsection{Proof of Lemma 3.2.2}

Observe that Lemma 3.2.1 and part [1] of the definition of SE together imply that for all $t, t^{\prime}$,

$$
c\left(\sigma\left(t^{\prime}\right), t\right)-c(\sigma(t), t) \geq \alpha\left(\sigma\left(t^{\prime}\right), t^{\prime}\right)-\alpha(\sigma(t), t) \geq c\left(\sigma\left(t^{\prime}\right), t^{\prime}\right)-c\left(\sigma(t), t^{\prime}\right) .
$$

We show the first claim. Consider first the case that $\alpha$ is strictly increasing in $t$. Suppose there exists $t$ such that $\sigma(t)<s^{*}(t)$. Since $s^{*}$ is continuous by Proposition 3.2.2 and is assumed to be weakly increasing (Assumption 3.2), we can take $t^{\prime}<t^{2}$ such that $\sigma(t)<s^{*}\left(t^{\prime}\right) \leq s^{*}(t)$. Then, we obtain

$$
\begin{aligned}
\alpha\left(\sigma\left(t^{\prime}\right), t^{\prime}\right)-c\left(\sigma\left(t^{\prime}\right), t^{\prime}\right) & <\alpha\left(\sigma(t), t^{\prime}\right)-c\left(\sigma(t), t^{\prime}\right) \\
& \leq \alpha(\sigma(t), t)-c\left(\sigma(t), t^{\prime}\right) .
\end{aligned}
$$

The first inequality holds due to $\sigma\left(t^{\prime}\right)<\sigma(t)<s^{*}\left(t^{\prime}\right)$ and Assumption 3.1. The second holds as we assume $\alpha$ to be increasing in $t$. This inequality contradicts (3.10), however. Hence, $\sigma(t) \geq s^{*}(t)$ for all $t$.

By a similar reasoning, we can obtain that $\sigma(t) \leq s^{*}(t)$ for all $t$ if $\alpha$ is strictly decreasing in $t$.

To see the second claim, suppose that $\sigma$ is discontinuous at $t$. Since $\sigma$ is a monotone function, both $\sigma(t-):=\lim _{x \uparrow t} \sigma(x)$ and $\sigma(t+):=\lim _{x \downarrow t} \sigma(x)$ exist. We will show that $\sigma(t-)=\sigma(t)=\sigma(t+)$.

Consider the case that $\alpha$ is strictly increasing in $t$. A similar reasoning can be applied in the other case. Since $\sigma$ is strictly increasing, $\sigma(t) \leq \sigma(t+)$. Letting $t^{\prime} \downarrow t$ in (3.10) we get

$$
c(\sigma(t+), t)-c(\sigma(t), t) \geq \alpha(\sigma(t+), t)-\alpha(\sigma(t), t) \geq c(\sigma(t+), t)-c(\sigma(t), t) .
$$

Thus, equality holds and by rearranging terms we get

$$
\alpha(\sigma(t), t)-c(\sigma(t), t)=\alpha(\sigma(t+), t)-c(\sigma(t+), t) .
$$

\footnotetext{
${ }^{2}$ Observe that it must be the case that $t>\underline{t}$ because $\sigma(\underline{t}) \in S \equiv[0,+\infty)$ and $s^{*}(\underline{t})=0$ by Assumption 3.
} 
Chapter 3. Separating Equilibria in One Dimensional Non-Pure Signaling Games

Since $s^{*}(t) \leq \sigma(t) \leq \sigma(t+)$, Assumption 3.1 implies $\sigma(t)=\sigma(t+)$.

We next verify that $\sigma(t-)=\sigma(t)$. Letting $t^{\prime} \uparrow t$ in (3.10) gives

$$
\alpha(\sigma(t), t)-c(\sigma(t), t)=\alpha(\sigma(t-), t)-c(\sigma(t-), t) .
$$

We first show that $\sigma(t-) \geq s^{*}(t)$. If not, there exists due to Assumption 3.2 and Proposition 3.2.2 a $t^{\prime}<t$ such that $\sigma(t-)<s^{*}\left(t^{\prime}\right) \leq s^{*}(t)$. Then $\sigma\left(t^{\prime}\right)<$ $s^{*}\left(t^{\prime}\right)$ because $\sigma\left(t^{\prime}\right) \leq \sigma(t-)$. This is, however, a contradiction to the first claim.

Thus, $s^{*}(t) \leq \sigma(t-) \leq \sigma(t)$, which due to Assumption 3.2 and (3.11) implies that $\sigma(t-)=\sigma(t)$.

\subsubsection{Proof of Lemma 3.2.3}

The proof of Lemma 3.2.3 is based on Lemmas 2.8.1, 2.8.2 and 2.8.3 from Appendix 2.8.1 in Chapter 2, which we repeat for the sake of completeness. Recall that $h_{\sigma}(t):=c_{s}(\sigma(t), t)$ for $t \in T$.

Lemma 3.5.1. Suppose that $\sigma$ is strictly increasing and $c$ has decreasing differences (Assumption 1.2). Then for any $t, t^{\prime} \in T$ with $t \neq t^{\prime}$,

$$
\operatorname{dist}_{\sigma}\left(t, t^{\prime}\right)=\inf _{\pi \in P\left(t, t^{\prime}\right)} l_{\sigma}(\pi) .
$$

Lemma 3.5.2. Let $\sigma$ be strictly increasing and continuous. Suppose Assumptions 1.2-4 hold. Then, for any partition $\pi$ from $t$ to $t^{\prime}$,

$$
L\left(\pi, h_{\sigma}, \sigma\right) \leq l_{\sigma}(\pi) \leq U\left(\pi, h_{\sigma}, \sigma\right) .
$$

Lemma 3.5.3. Let $\sigma$ be strictly increasing and continuous. Suppose that $c$ has decreasing differences. Then for all $t<t^{\prime}$ in $T$

$$
\sup _{\pi \in P\left(t, t^{\prime}\right)} L\left(\pi, h_{\sigma}, \sigma\right) \leq \operatorname{dist}_{\sigma}\left(t, t^{\prime}\right) .
$$

We are now ready to prove Lemma 3.2.3.

By Assumption 1.4, the function $h_{\sigma}$ is Riemann-Stieltjes integrable with respect to $\sigma$. By Lemmas 3.5.1 and 3.5.2,

$$
\int_{t}^{t^{\prime}} h_{\sigma}(x) d \sigma(x)=\inf _{\pi \in P\left(t, t^{\prime}\right)} U\left(\pi, h_{\sigma}, \sigma\right) \geq \inf _{\pi \in P\left(t, t^{\prime}\right)} l_{\sigma}(\pi)=\operatorname{dist}_{\sigma}\left(t, t^{\prime}\right) .
$$


On the other hand, by Lemma 3.5.3,

$$
\int_{t}^{t^{\prime}} h_{\sigma}(x) d \sigma(x)=\sup _{\pi \in P\left(t, t^{\prime}\right)} L\left(\pi, h_{\sigma}, \sigma\right) \leq \operatorname{dist}_{\sigma}\left(t, t^{\prime}\right) .
$$

Therefore, it follows

$$
\int_{t}^{t^{\prime}} h_{\sigma}(x) d \sigma(x)=\operatorname{dist}_{\sigma}\left(t, t^{\prime}\right)
$$

Finally, to prove uniqueness observe that for any $t, t^{\prime} \in T$

$$
\operatorname{dist}_{\sigma}\left(t, t^{\prime}\right)+\operatorname{dist}_{\sigma}\left(t^{\prime}, t\right)=\int_{t}^{t^{\prime}} h_{\sigma}(x) d \sigma(x)+\int_{t^{\prime}}^{t} h_{\sigma}(x) d \sigma(x)=0 .
$$

Thus, a node potential $p$ for $l_{\sigma}$ on $H$ is unique due to Fact 2 .

\subsubsection{Two Theorems Regarding the Initial Value Problem}

We show two general facts which are relevant to the initial value problem (3.6). Theorem 3.5.1 below addresses the existence of a solution while Theorem 3.5.2 does the uniqueness of a solution. The proof of the first theorem is loosely based on Peano's Theorem (See p.10 in Hartman (1982) for example).

Theorem 3.5.1. Let $V=\mathbb{R}_{+} \times \mathbb{R}$. Suppose $H: V \rightarrow \mathbb{R}$ is continuous and bounded. Then there exists at least one solution on $[\underline{s}, \infty)$ of the initial value problem

$$
f^{\prime}(s)=H(s, f(s)) \text { with } f(\underline{s})=c,
$$

where $\underline{s} \geq 0$ and $c$ is a constant. Moreover, any solution is automatically continuously differentiable.

Proof. We first set $\bar{s}=c=0$.

For $n, k \in \mathbb{N}$ define $s_{n, k}=k \cdot 2^{-n}$. Now take $n \in \mathbb{N}$ fixed for the moment. We set $x_{n, 0}=0$, and for $k \in \mathbb{N}$

$$
x_{n, k+1}=x_{n, k}+2^{-n} \cdot H\left(s_{n, k}, x_{n, k}\right) .
$$

Define $f_{n}: \mathbb{R}_{+} \rightarrow \mathbb{R}$ by, for $s \in\left[s_{n, k}, s_{n, k+1}\right)$,

$$
f_{n}(s)=x_{n, k}+2^{n} \cdot\left(s-s_{n, k}\right) \cdot\left(x_{n, k+1}-x_{n, k}\right) .
$$


Chapter 3. Separating Equilibria in One Dimensional Non-Pure Signaling Games

Let $M>0$ be such that $|H(s, a)| \leq M$ for all $(s, a) \in V$. First we show for all $s, s^{\prime} \in \mathbb{R}_{+}$that

$$
\left|f_{n}\left(s^{\prime}\right)-f_{n}(s)\right| \leq M \cdot\left|s^{\prime}-s\right| .
$$

Take $k, m$ such that $s \in\left[s_{n, k}, s_{n, k+1}\right)$ and $s^{\prime} \in\left[s_{n, m}, s_{n, m+1}\right)$. Then $f_{n}(s)$ is a linear interpolation between $x_{n, k}$ and $x_{n, k+1}$, and $f_{n}\left(s^{\prime}\right)$ is a linear interpolation between $x_{n, m}$ and $x_{n, m+1}$. Therefore it suffices to prove that

$$
\left|x_{n, k+1}-x_{n, k}\right| \leq M \cdot\left|s_{n, k+1}-s_{n, k}\right| .
$$

This however immediately follows from the observation that

$$
\begin{aligned}
\left|x_{n, k+1}-x_{n, k}\right| & =\left|2^{-n} \cdot H\left(s_{n, k}, x_{n, k}\right)\right| \\
& \leq 2^{-n} \cdot M \\
& =M \cdot\left|s_{n, k+1}-s_{n, k}\right| .
\end{aligned}
$$

Now write

$$
D=\left\{k \cdot 2^{-n} \mid k, n \in \mathbb{N}\right\} .
$$

Obviously $D$ is a countable set. Let $d_{1}, d_{2}, \ldots$ be an enumeration of $D$. Consider $d_{1} \in D$. Then for all $n$ we have $\left|f_{n}\left(d_{1}\right)\right| \leq M \cdot d_{1}$. So, there is a subsequence $\left(f_{n}^{1}\right)_{n=1}^{\infty}$ of $\left(f_{n}\right)_{n=1}^{\infty}$ for which $\left(f_{n}^{1}\left(d_{1}\right)\right)_{n=1}^{\infty}$ is convergent. Iteratively for $k=1,2, \ldots$ we can take a subsequence $\left(f_{n}^{k}\right)_{n=1}^{\infty}$ of $\left(f_{n}^{k-1}\right)_{n=1}^{\infty}$ for which $\left(f_{n}^{k}\left(d_{l}\right)\right)_{n=1}^{\infty}$ is convergent for all $l=1, \ldots k$. Then for the sequence $\left(f_{k}^{k}\right)_{k=1}^{\infty}$ we have that $\left(f_{k}^{k}(d)\right)_{k=1}^{\infty}$ is convergent for all $d \in D$.

Write $g_{k}=f_{k}^{k}$. Take any $s \geq 0$. We argue that $\left(g_{k}(s)\right)_{k=1}^{\infty}$ is Cauchy. Take $\varepsilon>0$. Since $D$ is dense in $\mathbb{R}_{+}$, we can take a $d \in D$ with $|s-d|<\frac{\varepsilon}{3 M}$. Further, since $\left(g_{k}(d)\right)_{k=1}^{\infty}$ is convergent, we can take $K>0$ such that

$$
\left|g_{k}(d)-g_{m}(d)\right|<\frac{\varepsilon}{3}
$$

whenever $k, m>K$. Then, for $k, m>K$,

$$
\begin{aligned}
\left|g_{k}(s)-g_{m}(s)\right| & =\left|g_{k}(s)-g_{k}(d)+g_{k}(d)-g_{m}(d)+g_{m}(d)-g_{m}(s)\right| \\
& \leq\left|g_{k}(s)-g_{k}(d)\right|+\left|g_{k}(d)-g_{m}(d)\right|+\left|g_{m}(d)-g_{m}(s)\right| \\
& <M \cdot|s-d|+\frac{\varepsilon}{3}+M \cdot|s-d| \\
& \leq M \cdot \frac{\varepsilon}{3 M}+\frac{\varepsilon}{3}+M \cdot \frac{\varepsilon}{3 M}=\varepsilon .
\end{aligned}
$$


Define $f(s)=\lim _{n \rightarrow \infty} g_{n}(s)$. We claim that $f$ is continuous. Take any $s, s^{\prime} \geq 0$. Since $\left(g_{n}\right)_{n=1}^{\infty}$ is a subsequence of $\left(f_{n}\right)_{n=1}^{\infty}$, we know from the argument above that

$$
\left|g_{n}\left(s^{\prime}\right)-g_{n}(s)\right| \leq M \cdot\left|s^{\prime}-s\right|
$$

for all $n$. The claim now follows by taking limits for $n \rightarrow \infty$.

So, the function $s \mapsto H(s, f(s))$ is continuous. Then it is integrable on bounded intervals. Take any $s \geq 0$. We show that

$$
f(s)=\int_{0}^{s} H(u, f(u)) d u .
$$

Take $n \in \mathbb{N}$ fixed. Let $p(n)$ be the natural number such that $g_{n}=f_{n}^{n}=f_{p(n)}$. Define

$$
h_{n}(s)=H\left(s_{p(n), k}, x_{p(n), k}\right) \quad \text { if } s \in\left[s_{p(n), k}, s_{p(n), k+1}\right) .
$$

Then clearly $g_{n}(s)=\int_{0}^{s} h_{n}(u) d y$. We argue that $h_{n}(s) \rightarrow H(s, f(s))$ as $n \rightarrow \infty$. First note that

$h_{n}\left(s_{p(n), k}\right)=H\left(s_{p(n), k}, x_{p(n), k}\right)=H\left(s_{p(n), k}, f_{p(n)}\left(s_{p(n), k}\right)\right)=H\left(s_{p(n), k}, g_{n}\left(s_{p(n), k}\right)\right)$.

Take $s \geq 0$ arbitrary. Choose $k(n)$ such that $s \in\left[s_{p(n), k(n)}, s_{p(n), k(n)+1}\right)$. Then

$$
h_{n}(s)=H\left(s_{p(n), k(n)}, x_{p(n), k(n)}\right)=H\left(s_{p(n), k(n)}, g_{n}\left(s_{p(n), k(n)}\right)\right) .
$$

Since clearly $p(n) \rightarrow \infty$ as $n \rightarrow \infty$, we know that $s_{p(n), k(n)} \rightarrow s$ as $n \rightarrow \infty$. Therefore it suffices to show that

$$
g_{n}\left(s_{p(n), k(n)}\right) \rightarrow f(s) .
$$

This however follows from the observations that $g_{n}(s) \rightarrow f(s)$ and $\mid g_{n}\left(s^{\prime}\right)-$ $g_{n}(s)|\leq M \cdot| s^{\prime}-s \mid$.

Hence, since $\left|h_{n}(s)\right| \leq M$ for all $s$ and $n$, the theorem of bounded convergence yields

$$
f(s)=\lim _{n \rightarrow \infty} g_{n}(s)=\lim _{n \rightarrow \infty} \int_{0}^{s} h_{n}(u) d u=\int_{0}^{s} H(u, f(u)) d u .
$$

Therefore, the desired result follows when $\underline{s}=c=0$. 
Chapter 3. Separating Equilibria in One Dimensional Non-Pure Signaling Games

Now, for arbitrary $\underline{s} \geq 0$ and $c$, we define the mapping $\tilde{H}: \mathbb{R}_{+} \times \mathbb{R} \rightarrow \mathbb{R}$ as

$$
\tilde{H}(s, a)=H(s+\underline{s}, a-c)
$$

and consider the initial value problem

$$
g^{\prime}(s)=\tilde{H}(s, g(s)) \text { with } g(0)=0 .
$$

Since $\tilde{H}$ is clearly continuous and bounded on $\mathbb{R}_{+} \times \mathbb{R}$, by the result obtained above, there exists a solution $g$ on $[0, \infty)$. Define next the function $f:[\underline{s}, \infty) \rightarrow \mathbb{R}$ as $f(s)=g(s-\underline{s})+c$. Then, $f(\underline{s})=c$ and for $s \in[\underline{s}, \infty)$

$$
f^{\prime}(s)=g^{\prime}(s-\underline{s})=\tilde{H}(s-\underline{s}, g(s-\underline{s}))=H(s, g(s-\underline{s})-c)=H(s, f(s)) .
$$

Hence, such $f$ is the desired solution. This completes the proof.

A subset $I$ of $S$ is called an initial subset if for all $s \in I$ and $s^{\prime} \in S$ with $s^{\prime}<s$ we have $s^{\prime} \in I$. A function $g: I \rightarrow \mathbb{R}$ is called a partial solution on $I$ if $g(\underline{s})=c, g$ is differentiable on $I$, and

$$
g^{\prime}(s)=H(s, g(s))
$$

for all $s \in I$.

Theorem 3.5.2. Let $V=\mathbb{R}_{+} \times \mathbb{R}$. Suppose that $H: V \rightarrow \mathbb{R}$ is continuous and non-increasing in the second coordinate. Then the initial value problem

$$
f^{\prime}(s)=H(s, f(s)) \text { with } f(0)=0
$$

has at most one solution $f$. Moreover, for any partial solution $g$ on $I$ it holds that $f(s)=g(s)$ for all $s \in I$.

Proof. Suppose that $f$ is a solution of the initial value problem, and that $g$ is a partial solution on $I$. It suffices to show that $f(s)=g(s)$ for all $s \in I$.

For $s \in I$, define $h(s)=f(s)-g(s)$. We show that $h(s)=0$ for all $s \in I$. Suppose that $h(s)>0$ for some $s \in I$. Since $h(0)=0$, obviously $s>0$. Define

$$
s^{*}=\inf \{x \mid 0 \leq x \leq s \text { and } h(y)>0 \text { for all } y \in(x, s)\} .
$$


Since $h$ is continuous, $h(s)>0$, and $h(0)=0$, we know that $s^{*}<s$ and $h\left(s^{*}\right)=$ 0 . So, since $h$ is continuously differentiable, by the mean value theorem there exists $\tau \in\left(s^{*}, s\right)$ with

$$
h^{\prime}(\tau)=\frac{h(s)-h\left(s^{*}\right)}{s-s^{*}}=\frac{h(s)}{s-s^{*}}>0 .
$$

However, $\tau \in\left(s^{*}, s\right)$ implies that $h(\tau)>0$ by definition of $s^{*}$. Then $f(\tau)>$ $g(\tau)$. So, since $H$ is non-increasing in the second argument, $f^{\prime}(\tau)=H(\tau, f(\tau)) \leq$ $H(\tau, g(\tau))=g^{\prime}(\tau)$. Hence, $h^{\prime}(\tau) \leq 0$. Contradiction. 



\section{Chapter 4}

\section{Separating Equilibria in Multidimensional Signaling}

\section{Games}

\subsection{Introduction}

This chapter offers a systematic approach to find a separating equilibrium (SE) in multidimensional pure signaling games. Such games cover natural extensions of the signaling games by Spence (1973). Most literature on signaling models has focused on one-dimensional signaling: both type and signal are assumed to be a scalar. But, it is hard to imagine, say in the job market model by Spence, how the characteristics of a job applicant can be fully captured by a single scalar. For example, in addition to the education level, extracurricular activities can be a signal to reveal the job applicant's social skill, a natural component of her total productivity. Considering the typical multiplicity of characteristics and signaling devices in the real world, it is important to know whether the results in one dimensional games can be extended to multidimensional contexts. Therefore, it is relevant and natural to extend signaling games to a setting with multidimensional types and signals.

We provide a characterization of a sender's strategy in SE of signaling games when type and signaling spaces are multidimensional. While the 
form of the sender's strategy in SE of single dimensional games is well understood in a fairly general setting (e.g., Mailath (1987), Mailath and von Thadden (2013) and Chapters 2, 3), our knowledge of those in multidimensional signaling games is limited. The main obstacle is that though types are multidimensional, the only incentive device at our disposal is one-dimensional monetary payment. Despite this obstacle, putting some constraints on the signaling cost function, we obtain necessary and sufficient conditions for the existence of SE in multidimensional games, which can be used in applications.

Our approach to determine the conditions uses the known results in single dimensional games and the ideas from traditional consumer theory. We first consider a single dimensional part of the original game by which we mean that its type set is a linearly ordered subset of the original type set. From an SE in this one dimensional game, which is relatively easy to find, we derive information about the signaling cost in SE of the original game. Exploiting this information and incentive compatibility between types that would under complete information induce the same response of the receiver allows us to fully determine the signals in SE. This step is reminiscent of the derivation of the Hicksian demand function from the expenditure function. From this procedure, we obtain necessary conditions of an SE which include that a sender's strategy is a solution to a certain standard optimization problem. These conditions are also sufficient for the sender's strategy in SE.

The chapter is organized as follows. In Section 4.2, we offer a literature review. In Section 4.3, we present the model and in Section 4.4 we offer a characterization of a sender's strategy in SE. In Section 4.5, we discuss the model of Quinzii and Rochet (1985) and show that some of their core results can be derived from our results as well. Section 4.6 concludes.

\subsection{Literature review}

The literature on multidimensional signaling games deals mainly with the unidimensional type, multidimensional signal case. The games in which both a type and a signal are multidimensional, which this chapter is concerned with, have been hardly studied. 


\subsubsection{Unidimensional type, multidimensional signal cases}

Milgrom and Roberts (1986) study a monopolistic firm's strategy in environments in which both the price and the level of advertising are a firm's decision variable (two dimensional signal) that may potentially be used as signals of quality of a good. The quality, which can be high or low, (unidimensional type) is unobservable because the good is newly released. They show that in equilibrium both the price and the advertising level can be used as signals differentiating between high- and low-quality firms, i.e., there is a separating equilibrium. Wilson (1985) studies a similar environment and shows that the result by Milgrom and Roberts can be extended to a more general formulation. In particular, he shows that in the case of a continuum of qualities, the necessary condition that the sender's strategy is separating is given as a certain differential equation.

Kim (2007) extends Spence's job market model. In his model, a job seeker is characterized by his productivity, which is private information. His productivity (unidimensional type) can be either high or low. The main difference from Spence's model is that before he enters the labor market, a job seeker can be involved with two kinds of activities, academic activity and social activity (two dimensional signal).

In the finance, Constantinides and Grundy (1989) recognize stock repurchase and financing as (two dimensional) signals in a firm's optimal investment problem. In their model, a sender is management who has (unidimensional) private information about the firm and a receiver is the collection of outside stockholders and the potential buyers of the new claim issued by the firm. Through the analysis of this signaling model, they highlight the signaling role of the chosen mode of financing and investigate how a stock repurchase, coupled with the issue of a senior security, permits management to signal its information to the market and accept a positive net present-value project.

\subsubsection{Multidimensional type, multidimensional signal cases}

The most closely related model to ours can be found in Quinzii and Rochet (1985). They study a multidimensional extension of Spence's job market model in which the dimension of a job seeker's type is the same as that of 
the signal. They offer an elegant characterization of separating equilibria as solutions of some partial differential equation. But, they assume that the signaling cost function is separable and linear and therefore the question how to find separating equilibria when cost functions have different forms still remains far from fully answered.

In biology, Johnstone (1995) applies a signaling game to study the issue of honesty in animal communication. Under the same setup as in Quinzii and Rochet (1985), he demonstrates that honest advertisement (or separating equilibrium in economic terms) of multidimensional qualities using multidimensional signals is evolutionary stable.

From the perspective of screening, ${ }^{1}$ Engers (1987) examines a market with asymmetric information where there are multidimensional signals and types. He proves under general conditions the existence of a Paretodominant separating set. A separating set consists of signal-price offers by a buyer (the uninformed party) such that if a seller (the informed party) each chooses their best offer from the set, then the buyer earns zero profits on each transaction. This is separating in the sense that products of different values are sold at different prices and thus are associated with different signal levels. The notion of separating set conceptually corresponds to separating equilibrium in signaling models.

\subsection{Model}

\subsubsection{Setup and notation}

We start with the description of the canonical signaling game. There are two players, called a sender and a receiver. We assume that the sender knows the value of some random variable $t$ whose support is a given set $T$. The sender, knowing the realization of the random variable $t$, sends to the receiver a signal $s$ in a set $S$. The receiver receives this signal, and

\footnotetext{
${ }^{1}$ In screening models, an uninformed party acts first, announcing signal-action offers to an informed party who then selects an optimal offer from all those announced. In signaling models, an informed party acts first by choosing the level of signal, and an uninformed party takes an action upon observing the signal. (See, e.g.,Bolton and Dewatripont (2005) and Kreps and Sobel (1994).)
} 
then takes an action $a$ in a set $A$. This ends the game and the payoffs are realized.

The payoff to the sender $U_{S}: A \times S \times T \rightarrow \mathbb{R}$ has the quasilinear form given as

$$
U_{S}(a, s, t)=a-c(s, t)
$$

where $c: S \times T \rightarrow \mathbb{R}_{+}$is a signaling cost of a type $t$ sender to deliver signal $s$. The payoff to the receiver is a mapping $U_{R}: A \times T \rightarrow \mathbb{R}$. Thus, the signaling games under consideration belong to the class of pure signaling games in the sense that the payoff of the receiver does not depend on the signal $s$. We assume that for any $t \in T, U_{R}(a, t)$ is maximized uniquely at $a=\alpha(t)$, that is,

$$
\{\alpha(t)\}=\arg \max _{a \in A} U_{R}(a, t) .
$$

We call the mapping $\alpha$ the best action of the receiver. This is the action the receiver would take under complete information.

Definition 4.3.1. A signaling game $\mathbf{G}=(S, T, A, c, \alpha)$ is a game of two players, a sender and a receiver, and specifies a space of signals $S$, a space of the sender's type $T$, a space of the receiver's action $A$, the sender's signaling cost function $c: S \times T \rightarrow \mathbb{R}$ and the best response of the receiver $\alpha: T \rightarrow A$. When $A=\mathbb{R}_{+}$, $S=\mathbb{R}_{+}^{n}$ and $T$ is an open convex cone in $\mathbb{R}_{+}^{m}$ of which the closure includes 0 , the game is denoted by $\mathbf{G}^{n, m}$.

Generic elements of $S$ and $T$ are denoted as $s=\left(s_{1}, \ldots, s_{n}\right)$ and $t=\left(t_{1}, \ldots, t_{m}\right)$, respectively. We put the following structural assumptions on the mappings $\alpha$ and $c$.

Assumption 4.3.1 (Monotone best action). For all $t \in T, \alpha(\lambda t)>\alpha(t)$ if $\lambda>1$ and $\lim _{\|t\| \rightarrow 0} \alpha(t)=0$.

Assumption 4.3.2 (Differentiable best action). The mapping $\alpha$ is in $C^{1}$, that is, first order partial derivatives exist and they are continuous.

Assumption 4.3.3 (Monotone cost in $s$ ). For all $s \neq 0, t, c(\lambda s, t)>c(s, t)$ if $\lambda>1$. And, $c(0, t)=0$ for all $t \in T$.

Assumption 4.3.4 (Monotone cost in $t$ ). For all $s \neq 0, c(s, t)$ is strictly decreasing in $t_{i},(i=1, \ldots, n)$. 
Assumption 4.3.5 (Differentiable cost). The mapping $c$ is in $C^{1}$.

Assumption 4.3.6 ( $g$-homogeneity). There exists a function $g:(0, \infty) \rightarrow(0, \infty)$ such that for all $(s, t) \in S \times T$ and $\lambda>0$,

$$
c(s, \lambda t)=g(\lambda) c(s, t)
$$

Assumptions 4.3.1, 4.3.3 and 4.3.4 are readily interpreted in the context of the job market model in which type $t$ represents an employee's productivity and the best response of the receiver $\alpha(t)$ is the wage an employer is willing to pay to an employee of type $t$. Assumption 4.3.6 is technical but general enough to include many classes of parametric cost functions. For instance, the CES (constant elasticity of substitution) function given as

$$
c(s, t)=\left(\sum_{i=1}^{n}\left(\frac{s_{i}}{t_{i}}\right)^{\rho}\right)^{1 / \rho}
$$

respects all assumptions with $g(\lambda)=1 / \lambda$.

It is worth mentioning how our assumptions on the cost function relate to Armstrong (1996). ${ }^{2}$ He considers a nonlinear pricing problem in which a consumer who consumes the bundle of goods $x \in \mathbb{R}^{n}$ enjoys the payoff $u(x, t)$ where $t \in \mathbb{R}^{m}$ is the consumer's type. Armstrong assumes that $u$ is increasing in all arguments, and $u$ is continuous, convex and homogenous of degree 1 in $t$ with $u(0, t)=u(x, 0)=0$. Except for the differentiability (Assumption 4.3.5), we put weaker constraints because we do not require $c$ is increasing in each $s_{i}$ and it is convex in $t$.

We end this subsection with basic, but useful observations about $g$.

Lemma 4.3.1. The function $g$ is continuous, strictly decreasing and $g(1)=$ 1. Moreover, $g$ is multiplicatively separable, i.e., $g\left(\lambda_{1} \lambda_{2}\right)=g\left(\lambda_{1}\right) g\left(\lambda_{2}\right)$ for all $\lambda_{1}, \lambda_{2}>0$.

Proof. Fix $(s, t) \in S \times T$ such that $c(s, t)>0$. This is possible due to Assumption 4.3.3.

${ }^{2}$ I am grateful to Dezsö Szalay for indicating the relation between our assumptions and Armstrong (1996). 
Take $\lambda_{1}>\lambda_{2}$ and $s \neq 0$. Then, we have

$$
\begin{aligned}
g\left(\lambda_{1}\right) c(s, t) & =c\left(s, \lambda_{1} t\right) \\
& <c\left(s, \lambda_{2} t\right) \\
& =g\left(\lambda_{2}\right) c(s, t),
\end{aligned}
$$

which implies $g\left(\lambda_{1}\right)<g\left(\lambda_{2}\right)$. Thus, $g$ is strictly decreasing.

To see continuity, take a sequence $\left\{\lambda_{n}\right\}$ converging to $\lambda$. Then, by Assumptions 4.3.5 and 4.3.6, it holds that as $n \rightarrow \infty$

$$
g\left(\lambda_{n}\right) c(s, t)=c\left(s, \lambda_{n} t\right) \rightarrow c(s, \lambda t)=g(\lambda) c(s, t)
$$

which implies that $g\left(\lambda_{n}\right) \rightarrow g(\lambda)$. Hence, $g$ is continuous.

Plugging $\lambda=1$ in (4.1) gives $g(1)=1$.

Lastly, $g$ is multiplicatively separable because

$$
g\left(\lambda_{1}\right) g\left(\lambda_{2}\right) c(s, t)=g\left(\lambda_{1}\right) c\left(s, \lambda_{2} t\right)=c\left(s, \lambda_{1} \lambda_{2} t\right)=g\left(\lambda_{1} \lambda_{2}\right) c(s, t) .
$$

The desired result follows.

\subsubsection{Incentive Compatibility of Separating equilibrium}

A (pure) strategy of the sender is a mapping $\sigma: T \rightarrow S$. A (pure) strategy of the receiver is a function $\gamma: S \rightarrow A$. Since in this paper we only focus on pure strategies, we omit the prefix "pure" from now on. Among possible equilibria, our attention goes to those in which information asymmetry resolves.

Definition 4.3.2. A strategy profile $(\sigma, \gamma)$ is a separating equilibrium (SE) if

1. $\sigma$ is one-to-one;

2. for all $t \in T$, we have

(a) $U_{S}(\gamma(\sigma(t)), \sigma(t), t) \geq U_{S}(\gamma(s), s, t)$ for all $s \in S$;

(b) $U_{R}(\gamma(\sigma(t)), t) \geq U_{R}(a, t)$ for all $a \in A$. 
If a strategy $\sigma$ of the sender is part of a separating equilibrium, it is called a separating equilibrium strategy (SE strategy). Note that when $(\sigma, \gamma)$ is an SE, it holds $\gamma(\sigma(t))=\alpha(t)$ because the receiver, upon observing $\sigma(t)$, infers the sender's type is $t$ and chooses the optimal action $\alpha(t)$ against the sender of type $t$. The following lemma allows us to focus only on the sender's strategy in analyzing an SE.

Lemma 4.3.2. A strategy $\sigma$ of the sender is an SE strategy if and only if $\sigma$ is one-to-one, $\lim _{\|t \mid\| \rightarrow 0} \sigma(t)=0$ and incentive compatibility (IC) holds, that is,

$$
\alpha\left(t^{\prime}\right)-\alpha(t) \leq c\left(\sigma\left(t^{\prime}\right), t\right)-c(\sigma(t), t)
$$

for all $t, t^{\prime} \in T$.

The first condition plays the role of an initial condition. The second one immediately comes from the definition of SE. The third, (IC), means that for type $t$, the additional benefit of mimicking $t^{\prime}$, the left hand side, is not larger than the additional cost of mimicking, the right hand side.

Proof. We show both implications separately.

A. Let $(\sigma, \gamma)$ be an SE. Since $\sigma$ is separating, it is one-to-one by definition.

To see (IC), observe that since $(\gamma \circ \sigma)(t)=\alpha(t)$ for all $t$,

$$
\alpha(t)-c(\sigma(t), t)=(\gamma \circ \sigma)(t)-c(\sigma(t), t) \geq \gamma(s)-c(s, t)
$$

for all $s$. The inequality holds because $\sigma$ is a best response to $\gamma$.

Substitution of $s=\sigma\left(t^{\prime}\right)$ and rewriting then yields

$$
\alpha\left(t^{\prime}\right)-\alpha(t) \leq c\left(\sigma\left(t^{\prime}\right), t\right)-c(\sigma(t), t) .
$$

Thus, (IC) follows.

Lastly, observe that for all $s$ and $t$,

$$
\alpha(t)-c(\sigma(t), t) \geq \gamma(s)-c(s, t) \geq-c(s, t)
$$

where the first inequality is due to the definition of SE (Definition 4.3.2) and the second due to the fact that $\gamma(s) \in A \equiv \mathbb{R}_{+}$. 
By taking $s=0$ in (4.2), by Assumption 4.3.3 we obtain that for all $t$

$$
\alpha(t) \geq c(\sigma(t), t) \geq 0 .
$$

Thus, since $\lim _{\| t|| \rightarrow 0} \alpha(t)=0$ by Assumption 4.3.1, it holds $\lim _{\|t\| \rightarrow 0} c(\sigma(t), t)=$ 0. By Assumption 4.3.3, it is the case that $\lim _{\|t \mid\| \rightarrow 0} \sigma(t)=0$.

B. Conversely, suppose $\sigma$ is one to one, $\lim _{\|t\| \rightarrow 0} \sigma(t)=0$ and satisfies IC. Since $\sigma$ is one-to-one, for every $s \in S$ there is at most one $t \in T$ with $\sigma(t)=s$. So, we can define the strategy $\gamma$ of the receiver by

$$
\gamma(s)=\left\{\begin{aligned}
\alpha(t) & \text { if } t \in T \text { is such that } \sigma(t)=s \\
0 & \text { otherwise. }
\end{aligned}\right.
$$

It suffices to show that $(\sigma, \gamma)$ is a Nash equilibrium.

First, for all $a \in A$

$$
U_{R}(\gamma(\sigma(t)), t)=U_{R}(\alpha(t), t) \geq U_{R}(a, t)
$$

by definition of $\gamma$ and $\alpha$. So, $\gamma$ is a best response of the receiver to $\sigma$.

Second, suppose the sender is of type $t$. Take $s \in S$.

1. If there exists $t^{\prime} \in T$ with $\sigma\left(t^{\prime}\right)=s$, then $\gamma(s)=\alpha\left(t^{\prime}\right)$. So, by the construction of $\gamma$ and the condition IC,

$$
U_{S}(\gamma(\sigma(t)), \sigma(t), t)=\alpha(t)-c(\sigma(t), t) \geq \alpha\left(t^{\prime}\right)-c\left(\sigma\left(t^{\prime}\right), t\right)=U_{S}(\gamma(s), s, t) .
$$

2. Otherwise, $\gamma(s)=0$. In this case, we take any sequence $\left\{t_{n}\right\}$ in $T$ which converges to $\mathbf{0}$. Then, it holds that

$$
\begin{aligned}
U_{S}(\gamma(\sigma(t)), \sigma(t), t) & =\alpha(t)-c(\sigma(t), t) & \\
& \geq \alpha\left(t_{n}\right)-c\left(\sigma\left(t_{n}\right), t\right) & (\mathrm{by}(\mathrm{IC})) \\
& \rightarrow-\lim _{n \rightarrow \infty} c\left(\sigma\left(t_{n}\right), t\right) . & (\text { as } n \text { goes to infinity) }
\end{aligned}
$$

In addition, $U_{S}(\gamma(s), s, t)=0-c(s, t) \leq 0$. Hence, since $\lim _{n \rightarrow \infty} c\left(\sigma\left(t_{n}\right), t\right)=$ $\lim _{\left\|s^{\prime}\right\| \rightarrow 0} c\left(s^{\prime}, t\right)=0$, we obtain that

$$
U_{S}(\gamma(\sigma(t)), \sigma(t), t) \geq 0 \geq U_{S}(\gamma(s), s, t) .
$$

Therefore, $\sigma$ is a best response for the sender to $\gamma$. 


\subsection{Results}

We offer a characterization of an SE strategy in $\mathbf{G}^{n, m}=(S, T, A, c, \alpha)$. Our approach takes two steps. In the first step, we fix type $t$ and consider a one-dimensional signaling game of which the type set is given as the ray emanating from the origin and passing through $t$. Since this game is onedimensional, we can explicitly derive a unique SE strategy in this game without difficulty. Also, we are able to represent an SE strategy in that one-dimensional signaling game in terms of an SE strategy of the original game $\mathbf{G}^{n, m}$. Then, by exploiting the fact that the SE strategy in the onedimensional signaling game under consideration is unique, we obtain a certain condition that the signal for type $t$ in SE of $\mathbf{G}^{n, m}$ has to respect.

The second step is to take advantage of incentive compatibility between types that induce the same response from the receiver and to use the information obtained in the first step. We then can fully determine type $t^{\prime}$ s signal in SE of $\mathbf{G}^{n, m}$.

We give a formal analysis. Suppose that $\sigma: T \rightarrow S$ is an SE strategy in $\mathbf{G}^{n, m}$. Fix type $t$. Consider the one-dimensional signaling game

$$
\mathbf{G}_{t}^{1,1}=(\bar{S}, \bar{T}, A, \bar{c}, \bar{\alpha})
$$

where $\bar{S}=\mathbb{R}_{+}, \bar{T}=\mathbb{R}_{++}, A=\mathbb{R}_{+}$and

$$
\bar{c}(x, \lambda)=x \cdot g(\lambda), \text { and } \bar{\alpha}(\lambda)=\alpha(\lambda t)
$$

where the mapping $g$ comes from Assumption 4.3.6.

We derive a unique SE strategy of $\mathbf{G}_{t}^{1,1}$ in an explicit form.

Lemma 4.4.1. Let $t \in T$. The mapping $y: \bar{T} \rightarrow \bar{S}$ defined as

$$
y(\lambda)=\int_{0}^{\lambda} \frac{t}{g(z)} \cdot \nabla \alpha(z t) d z
$$

is a unique SE strategy in $\mathbf{G}_{t}^{1,1}$. Here, $\nabla$ denotes the gradient.

Proof. We first verify that the integral in (4.3) $\int_{0}^{\lambda} \frac{t}{g(z)} \cdot \nabla \alpha(z t) d z$ exists for all 
$\lambda>0$. Fix $\lambda>0$. Then, we have

$$
\begin{aligned}
\int_{0}^{\lambda} \frac{t}{g(z)} \cdot \nabla \alpha(z t) d z & \leq \int_{0}^{\lambda} \frac{t}{g(\lambda)} \cdot \nabla \alpha(z t) d z \\
& =\frac{1}{g(\lambda)} \int_{0}^{\lambda} \frac{d \alpha(z t)}{d z} d z \\
& =\frac{1}{g(\lambda)}\left(\alpha(\lambda t)-\lim _{z \rightarrow 0} \alpha(z t)\right) \\
& =\frac{1}{g(\lambda)} \cdot \alpha(\lambda t) \\
& <\infty .
\end{aligned}
$$

The first inequality is due to the fact that $g(z) \geq g(\lambda)>0$ for $z \leq \lambda$ by Lemma 4.3.1. Hence, the integral exists.

We claim that the mapping $y$ in (4.3) is an SE strategy using Lemma 4.3.2.

First, it is clear that $\lim _{\lambda \rightarrow 0} y(\lambda)=0$.

Second, the mapping $y$ is strictly increasing and thus one-to-one because the integrand in (4.3) is strictly positive. To see this, note that by Assumptions 4.3.1, the mapping $z \mapsto \alpha(z t)$ is strictly increasing and thus its derivative $t \cdot \nabla \alpha(z t)>0$ and that $g>0$ by Assumption 4.3.6.

Lastly, (IC) holds because

$$
\begin{aligned}
\bar{c}\left(y\left(\lambda^{\prime}\right), \lambda\right)-\bar{c}(y(\lambda), \lambda) & =\left(y\left(\lambda^{\prime}\right)-y(\lambda)\right) g(\lambda) \\
& =\int_{\lambda}^{\lambda^{\prime}} g(\lambda) \frac{t}{g(z)} \cdot \nabla \alpha(z t) d z \\
& \geq \int_{\lambda}^{\lambda^{\prime}} t \cdot \nabla \alpha(z t) d z \\
& =\int_{\lambda}^{\lambda^{\prime}} \frac{d \alpha(z t)}{d z} d z \\
& =\alpha\left(\lambda^{\prime} t\right)-\alpha(\lambda t) \\
& =\bar{\alpha}\left(\lambda^{\prime}\right)-\bar{\alpha}(\lambda) .
\end{aligned}
$$

To see the sole inequality, notice that if $\lambda^{\prime} \geq \lambda$, then $g(\lambda) / g(z) \geq 1$ for all $z \in\left[\lambda, \lambda^{\prime}\right]$ since $g$ is decreasing by Lemma 4.3.1. If $\lambda^{\prime} \leq \lambda$, then $g(\lambda) / g(z) \leq 1$ 
for all $z \in\left[\lambda^{\prime}, \lambda\right]$ and thus

$$
\begin{aligned}
\int_{\lambda}^{\lambda^{\prime}} g(\lambda) \frac{t}{g(z)} \cdot \nabla \alpha(z t) d z & =-\int_{\lambda^{\prime}}^{\lambda} g(\lambda) \frac{t}{g(z)} \cdot \nabla \alpha(z t) d z \\
& \geq-\int_{\lambda^{\prime}}^{\lambda} t \cdot \nabla \alpha(z t) d z=\int_{\lambda}^{\lambda^{\prime}} t \cdot \nabla \alpha(z t) d z .
\end{aligned}
$$

Therefore, we conclude that $y$ is an SE strategy by Lemma 4.3.2.

Finally, we show uniqueness. Suppose a mapping $\tilde{y}: \bar{T} \rightarrow \bar{S}$ is an SE strategy. Then it satisfies (IC):

$$
\begin{aligned}
& \bar{\alpha}\left(\lambda^{\prime}\right)-\bar{\alpha}(\lambda) \leq \bar{c}\left(\tilde{y}\left(\lambda^{\prime}\right), \lambda\right)-\bar{c}(\tilde{y}(\lambda), \lambda)=\left(\tilde{y}\left(\lambda^{\prime}\right)-\tilde{y}(\lambda)\right) g(\lambda) \\
& \bar{\alpha}(\lambda)-\bar{\alpha}\left(\lambda^{\prime}\right) \leq \bar{c}\left(\tilde{y}(\lambda), \lambda^{\prime}\right)-\bar{c}\left(\tilde{y}\left(\lambda^{\prime}\right), \lambda^{\prime}\right)=\left(\tilde{y}(\lambda)-\tilde{y}\left(\lambda^{\prime}\right)\right) g\left(\lambda^{\prime}\right)
\end{aligned}
$$

which together imply that

$$
\frac{\bar{\alpha}\left(\lambda^{\prime}\right)-\bar{\alpha}(\lambda)}{g(\lambda)} \leq \tilde{y}\left(\lambda^{\prime}\right)-\tilde{y}(\lambda) \leq \frac{\bar{\alpha}\left(\lambda^{\prime}\right)-\bar{\alpha}(\lambda)}{g\left(\lambda^{\prime}\right)} .
$$

Suppose $\lambda^{\prime}>\lambda$. Dividing by $\lambda^{\prime}-\lambda$ yields

$$
\frac{1}{g(\lambda)} \frac{\bar{\alpha}\left(\lambda^{\prime}\right)-\bar{\alpha}(\lambda)}{\left(\lambda^{\prime}-\lambda\right)} \leq \frac{\tilde{y}\left(\lambda^{\prime}\right)-\tilde{y}(\lambda)}{\lambda^{\prime}-\lambda} \leq \frac{1}{g\left(\lambda^{\prime}\right)} \frac{\bar{\alpha}\left(\lambda^{\prime}\right)-\bar{\alpha}(\lambda)}{\left(\lambda^{\prime}-\lambda\right)} .
$$

Letting $\lambda^{\prime} \searrow \lambda$, we have

$$
\frac{1}{g(\lambda)} \frac{d \bar{\alpha}(\lambda)}{d \lambda} \leq \lim _{\lambda^{\prime} \backslash \lambda} \frac{\tilde{y}\left(\lambda^{\prime}\right)-\tilde{y}(\lambda)}{\lambda^{\prime}-\lambda} \leq \frac{1}{g(\lambda)} \frac{d \bar{\alpha}(\lambda)}{d \lambda}
$$

that is,

$$
\lim _{\lambda^{\prime} \backslash \lambda} \frac{\tilde{y}\left(\lambda^{\prime}\right)-\tilde{y}(\lambda)}{\lambda^{\prime}-\lambda}=\frac{1}{g(\lambda)} \frac{d \bar{\alpha}(\lambda)}{d \lambda}
$$

In a similar way, we can show that

$$
\lim _{\lambda^{\prime} \nearrow \lambda} \frac{\tilde{y}\left(\lambda^{\prime}\right)-\tilde{y}(\lambda)}{\lambda^{\prime}-\lambda}=\frac{1}{g(\lambda)} \frac{d \bar{\alpha}(\lambda)}{d \lambda}
$$

Hence, $\tilde{y}$ is differentiable with its derivative

$$
\tilde{y}^{\prime}(\lambda)=\frac{1}{g(\lambda)} \frac{d \bar{\alpha}(\lambda)}{d \lambda}=\frac{t}{g(\lambda)} \cdot \nabla \alpha(\lambda t) .
$$


In addition, $\lim _{\lambda \rightarrow 0} \tilde{y}(\lambda)=0$ by Lemma 4.3.2. This means that for all $\lambda>0$

$$
\tilde{y}(\lambda)=\int_{0}^{\lambda} \frac{t}{g(z)} \cdot \nabla \alpha(z t) d z .
$$

Therefore, $y(\lambda)=\tilde{y}(\lambda)$ for all $\lambda>0$. The desired result follows.

On the other hand, the following lemma states that an SE strategy in $\mathbf{G}_{t}^{1,1}$ can also be written in terms of an SE strategy in $\mathbf{G}^{n, m}$.

Lemma 4.4.2. Suppose $\sigma$ is an SE strategy in $\mathbf{G}^{n, m}$. Let $t \in T$. Then, the function $x: \bar{T} \rightarrow \bar{S}$ defined as

$$
x(\lambda)=c(\sigma(\lambda t), t)
$$

is an SE strategy in $\mathbf{G}_{t}^{1,1}$.

Proof. We use Lemma 4.3.2. First, we have

$$
\begin{array}{rlr}
\lim _{\lambda \rightarrow 0} x(\lambda) & =\lim _{\lambda \rightarrow 0} c(\sigma(\lambda t), t) & \\
& =c\left(\lim _{\lambda \rightarrow 0} \sigma(\lambda t), t\right) & \text { (by Assumption 4.3.5) } \\
& =0 \quad \text { (by Lemma 4.3.2 and Assumption 4.3.3) }
\end{array}
$$

Second, we show the mapping $x$ is one to one. Suppose $\lambda^{\prime}>\lambda$. Then, it holds

$$
\begin{aligned}
0 & <\alpha\left(\lambda^{\prime} t\right)-\alpha(\lambda t) \\
& \leq c\left(\sigma\left(\lambda^{\prime} t\right), \lambda t\right)-c(\sigma(\lambda t), \lambda t) \\
& =g(\lambda) \cdot\left(c\left(\sigma\left(\lambda^{\prime} t\right), t\right)-c(\sigma(\lambda t), t)\right) .
\end{aligned}
$$$$
\text { (by (IC) in } \mathbf{G}^{n, m} \text { ) }
$$

(by Assumption 4.3.6)

Hence, $c\left(\sigma\left(\lambda^{\prime} t\right), t\right)>c(\sigma(\lambda t), t)$, which implies that the mapping $x$ is strictly increasing and thus one to one.

We lastly verify that the mapping $x$ respects (IC) in $\mathbf{G}_{t}^{1,1}$. This holds by the following observation:

$$
\begin{aligned}
\bar{\alpha}\left(\lambda^{\prime}\right)-\bar{\alpha}(\lambda) & =\alpha\left(\lambda^{\prime} t\right)-\alpha(\lambda t) \\
& \leq c\left(\sigma\left(\lambda^{\prime} t\right), \lambda t\right)-c(\sigma(\lambda t), \lambda t) \\
& =g(\lambda) \cdot x\left(\lambda^{\prime}\right)-g(\lambda) \cdot x(\lambda) \\
& =\bar{c}\left(x\left(\lambda^{\prime}\right), \lambda\right)-\bar{c}(x(\lambda), \lambda) .
\end{aligned}
$$

Therefore, $x$ is an SE strategy in $\mathbf{G}_{t}^{1,1}$ by Lemma 4.3.2. 
Putting together Lemmas 4.4.1 and 4.4.2, we obtain the following result.

Proposition 4.4.1. Suppose $\sigma$ is an SE strategy in $\mathbf{G}^{n, m}$. Then, for all $t \in T$ and $\lambda>0$,

$$
c(\sigma(\lambda t), t)=\int_{0}^{\lambda} \frac{t}{g(z)} \cdot \nabla \alpha(z t) d z
$$

Proof. It immediately follows from Lemmas 4.4.1 and 4.4.2 that $x(\lambda)=y(\lambda)$.

Proposition 4.4.1 gives the signaling expenditure of a type $t$ sender in SE by setting $\lambda=1$.

To address a characterization of an SE strategy, it is convenient to define two functions and a set for the ease of exposition.

Define the mapping $f: T \rightarrow \mathbb{R}$ by

$$
f(t):=\int_{0}^{1} \frac{t}{g(z)} \cdot \nabla \alpha(z t) d z
$$

that is, $f(t)$ is the cost of signaling at type $t$ in SE, and $F: S \times T \rightarrow \mathbb{R}$ by

$$
F(s, t):=c(s, t)-f(t) .
$$

We introduce the following notation.

Notation 4.4.1. We write $t^{\prime} \sim_{\alpha} t$ if $\alpha(t)=\alpha\left(t^{\prime}\right)$.

For fixed $t \in T$, we define the set

$$
U_{t}:=\bigcap_{\left\{t^{\prime}: t^{\prime} \sim \alpha\right.}\left\{s \in S: F\left(s, t^{\prime}\right) \geq 0\right\} .
$$

Observe that $U_{t}$ is closed because it is an intersection of closed sets and that $U_{\tilde{t}}=U_{t}$ for any $\tilde{t} \sim_{\alpha} t$.

The following theorem is the main result of this chapter.

Theorem 4.4.1. The mapping $\sigma: T \rightarrow S$ is an SE strategy in $\mathbf{G}^{n, m}$ if and only if it satisfies

C1. $\sigma$ is one-to-one; 
C2. $c(\sigma(t), t)=f(t)$ for all $t \in T$;

C3. For all $t \in T$,

$$
\sigma(t) \in \arg \min _{s \in U_{t}} c(s, t) .
$$

Proof. $(\Longrightarrow)$ Suppose that $\sigma$ is an SE strategy. C1 comes from the definition of SE. C2 is simply Proposition 4.4.1 when $\lambda=1$.

To verify C3, fix $t \in T$. We first claim that $\sigma(t) \in U_{t}$. For any type $t^{\prime} \in T$, (IC) implies that

$$
\alpha\left(t^{\prime}\right)-c\left(\sigma\left(t^{\prime}\right), t^{\prime}\right) \geq \alpha(t)-c\left(\sigma(t), t^{\prime}\right)
$$

In particular, for any $t^{\prime} \in T$ with $t^{\prime} \sim_{\alpha} t$, we then have

$$
c\left(\sigma(t), t^{\prime}\right) \geq c\left(\sigma\left(t^{\prime}\right), t^{\prime}\right)
$$

By Proposition 4.4.1, $c\left(\sigma\left(t^{\prime}\right), t^{\prime}\right)=f\left(t^{\prime}\right)$. Thus, (4.6) is rewritten as

$$
c\left(\sigma(t), t^{\prime}\right) \geq f\left(t^{\prime}\right)
$$

or equivalently

$$
F\left(\sigma(t), t^{\prime}\right) \geq 0 .
$$

The above inequality holds for all $t^{\prime} \in T$ such that $t^{\prime} \sim_{\alpha} t$. Hence,

$$
\sigma(t) \in \bigcap_{\left\{t^{\prime}: t^{\prime} \sim \alpha t\right\}}\left\{s \in S: F\left(s, t^{\prime}\right) \geq 0\right\} \equiv U_{t} .
$$

We now claim that $c(\sigma(t), t) \leq c(s, t)$ for all $s \in U_{t}$. Suppose there exists $s \in U_{t}$ such that $c(s, t)<c(\sigma(t), t)$. Then, we have

$$
\begin{aligned}
F(s, t) & =c(s, t)-f(t) \\
& <c(\sigma(t), t)-f(t) \\
& =0 .
\end{aligned}
$$

This, however, contradicts that $s \in U_{t}$. Therefore, C3 follows.

$(\Leftarrow=) \quad$ Suppose $\sigma$ satisfies $\mathrm{C} 1$ to $\mathrm{C} 3$. To show $\sigma$ is an SE strategy, we use Lemma 4.3.2.

First, the one-to-one property is simply $\mathrm{C} 1$. 
Second, we check that $\lim _{\|t\| \rightarrow 0} \sigma(t)=0$. Observe

$$
\begin{array}{rlr}
f(t) & =\int_{0}^{1} \frac{t}{g(z)} \cdot \nabla \alpha(z t) d z & \\
& \leq \int_{0}^{1} t \cdot \nabla \alpha(z t) d z & \text { (because } g(z) \geq 1 \text { for } z \leq 1 \text { by Lemma 4.3.1) } \\
& =\left.\lim _{y \rightarrow 0} \alpha(z t)\right|_{y} ^{1} & \\
& =\alpha(t) . & \text { (by Assumption 4.3.1) }
\end{array}
$$

Since $c(\sigma(t), t)-f(t)=0$ by C2, using $f(t) \leq \alpha(t)$ and Assumption 4.3.1,

$$
\lim _{\|t\| \rightarrow 0} c(\sigma(t), t)=\lim _{\|t\| \rightarrow 0} f(t) \leq \lim _{\|t\| \rightarrow 0} \alpha(t)=0 .
$$

Choose $\hat{t}$ such that each element $\hat{t}_{i}$ is sufficiently large $(i=1, \ldots, m)$. Then, by Assumption 4.3.4

$$
0 \leq \lim _{\|t\| \rightarrow 0} c(\sigma(t), \hat{t}) \leq \lim _{\|t\| \rightarrow 0} c(\sigma(t), t)=0
$$

that is,

$$
\lim _{\|t\| \rightarrow 0} c(\sigma(t), \hat{t})=0
$$

By Assumption 4.3.3, we get $\lim _{\|t \mid\| \rightarrow 0} \sigma(t)=0$.

Lastly, we verify (IC). Fix $t, t^{\prime} \in T$ and take $\lambda>0$ such that $\lambda t \sim_{\alpha} t^{\prime}$. This is possible because $\alpha$ is assumed to be continuous (Assumption 4.3.2). We first prove the following claim.

Claim 4.4.1. $c\left(\sigma\left(t^{\prime}\right), t\right) \geq \int_{0}^{\lambda} \frac{t}{g(z)} \cdot \nabla \alpha(z t) d z$

Proof. Notice that by C3

$$
c\left(\sigma\left(t^{\prime}\right), \lambda t\right) \geq c(\sigma(\lambda t), \lambda t)
$$


because $\sigma\left(t^{\prime}\right) \in U_{t^{\prime}}$ by C3 and $U_{t^{\prime}}=U_{\lambda t}$. Then, it holds

$$
\begin{aligned}
& c\left(\sigma\left(t^{\prime}\right), t\right)=\frac{1}{g(\lambda)} c\left(\sigma\left(t^{\prime}\right), \lambda t\right) \\
& \geq \frac{1}{g(\lambda)} c(\sigma(\lambda t), \lambda t) \\
& =\frac{1}{g(\lambda)} f(\lambda t) \\
& =\frac{1}{g(\lambda)} \int_{0}^{1} \frac{\lambda t}{g(y)} \cdot \nabla \alpha(y \lambda t) d y \\
& =\frac{1}{g(\lambda)} \int_{0}^{\lambda} \frac{t}{g(z / \lambda)} \cdot \nabla \alpha(z t) d z \quad \text { (by change of variable } z=\lambda y \text { ) } \\
& \left.=\int_{0}^{\lambda} \frac{t}{g(z)} \cdot \nabla \alpha(z t) d z \quad \quad \text { (since } g\left(\lambda_{1}\right) g\left(\lambda_{2}\right)=g\left(\lambda_{1} \lambda_{2}\right)\right)
\end{aligned}
$$

Hence, the desired result follows.

Thus, we have

$$
\begin{aligned}
& c\left(\sigma\left(t^{\prime}\right), t\right)-c(\sigma(t), t) \geq \int_{0}^{\lambda} \frac{t}{g(y)} \cdot \nabla \alpha(y t) d y-\int_{0}^{1} \frac{t}{g(y)} \cdot \nabla \alpha(y t) d y \\
& \text { (by the above claim and C2) }
\end{aligned}
$$

that is, (IC) holds.

Therefore, we conclude that $\sigma$ is an SE strategy.

It is worth noting that the minimization problem (4.5) in C3 resembles the derivation of the Hicksian demand function in traditional consumer theory, namely, the demand of goods that minimizes total expenditure required to reach a given level of utility. Interestingly, the SE strategy in signaling games conceptually corresponds to the Hicksian demand function in consumer theory. 
The significance of Theorem 4.4.1 is that an SE strategy can be obtained simply by solving a certain standard optimization problem. This task is not so difficult, especially compared to the partial differential equation approach by (Quinzii and Rochet, 1985). Indeed, Theorem 4.4.1 suggests the following procedure to compute an SE strategy.

1. Construct the set $U_{t}$ from the pair of given functions $c$ and $\alpha$. If empty, no SE exists.

2. Solve $\sigma(t) \in \arg \min _{s \in U_{t}} c(s, t)$ if $U_{t}$ is nonempty.

3. Check whether the solution of the optimization problem $\sigma(t)$ satisfies $\mathrm{C} 1$ and $\mathrm{C} 2$ as well. If so, it is an SE strategy. Otherwise, no SE exists.

The following example illustrates this procedure.

Example 4.4.1. We consider the signaling game $\mathbf{G}^{2,2}$ in which the cost function is linear in signal $s$ :

$$
c(s, t)=\frac{s_{1}}{t_{1}}+\frac{s_{2}}{t_{2}}
$$

and the best response of the receiver $\alpha$ is given as the CES (constant elasticity of substitution) function

$$
\alpha(t)=\left(\frac{1}{2} t_{1}^{\rho}+\frac{1}{2} t_{2}^{\rho}\right)^{1 / \rho} \text { with } \rho \leq 1 .
$$

In Step 1, we construct the set $U_{t}$. Note that

$$
f(t)=\int_{0}^{1} y t \cdot \nabla \alpha(y t) d y=\alpha(t) \int_{0}^{1} y d y=\frac{\alpha(t)}{2} .
$$

and so $F(s, t)=c(s, t)-f(t)=\frac{s_{1}}{t_{1}}+\frac{s_{2}}{t_{2}}-\frac{\alpha(t)}{2}$. Thus,

$$
\begin{aligned}
U_{t} & =\bigcap_{\left\{t^{\prime}: t^{\prime} \sim \alpha t\right\}}\left\{s \in S \mid \frac{s_{1}}{t_{1}^{\prime}}+\frac{s_{2}}{t_{2}^{\prime}}-\frac{\alpha\left(t^{\prime}\right)}{2} \geq 0\right\} \\
& =\left\{s \in S: \inf _{\left\{t^{\prime}: t^{\prime} \sim \alpha\right.}\left(\frac{s_{1}}{t_{1}^{\prime}}+\frac{s_{2}}{t_{2}^{\prime}}\right) \geq \frac{\alpha(t)}{2}\right\} \\
& =\left\{s \in S: s_{1}^{\rho /(1+\rho)}+s_{2}^{\rho /(1+\rho)} \geq \frac{\left(t_{1}^{\rho}+t_{2}^{\rho}\right)^{2 /(1+\rho)}}{2}\right\} .
\end{aligned}
$$


Since $U_{t}$ is not empty, we go to Step 2 . We solve

$$
\min _{s \in U_{t}}\left(\frac{s_{1}}{t_{1}}+\frac{s_{2}}{t_{2}}\right) .
$$

1. When $\rho \in(-1,0) \cup(0,1]$, the minimizer $\sigma(t)$ of $(4.10)$ is

$$
\sigma(t)=\left(\frac{t_{1}^{1+\rho}}{2^{(1+\rho) / \rho}}\left(t_{1}^{\rho}+t_{2}^{\rho}\right)^{(1-\rho) / \rho}, \frac{t_{2}^{1+\rho}}{2^{(1+\rho) / \rho}}\left(t_{1}^{\rho}+t_{2}^{\rho}\right)^{(1-\rho) / \rho}\right) .
$$

We check whether $\sigma$ satisfies $\mathrm{C} 1$ and C2. It is readily verified that $\sigma$ is one to one and $\mathrm{C} 1$ holds. $\mathrm{C} 2$ is also respected because for all $t$ $c(\sigma(t), t)=t_{1}^{-1} \cdot \frac{1}{2^{(1+\rho) / \rho}} \frac{t_{1}^{1+\rho}}{\left(t_{1}^{\rho}+t_{2}^{\rho}\right)^{1-1 / \rho}}+t_{2}^{-1} \cdot \frac{1}{2^{(1+\rho) / \rho}} \frac{t_{2}^{1+\rho}}{\left(t_{1}^{\rho}+t_{2}^{\rho}\right)^{1-1 / \rho}}=\frac{\alpha(t)}{2}=f(t)$.

Therefore, we conclude that $\sigma$ is the SE strategy by Theorem 4.4.1.

2. When $\rho<-1$, the minimizer $\sigma(t)$ of (4.10) is given as the corner solution

$$
\sigma(t)= \begin{cases}\left(0, \frac{1}{2^{(1+\rho) / \rho}}\left(t_{1}^{\rho}+t_{2}^{\rho}\right)^{\frac{2}{\rho}}\right) & \text { if } t_{1} \geq t_{2} \\ \left(\frac{1}{2^{(1+\rho) / \rho}}\left(t_{1}^{\rho}+t_{2}^{\rho}\right)^{\frac{2}{\rho}}, 0\right) & \text { if } t_{1} \leq t_{2}\end{cases}
$$

Hence, $\sigma$ is not one-to-one and thus there exits no SE. In fact, Quinzii and Rochet have proved the nonexistence of SE when $\rho=-2$ (Proposition 2 in Quinzii and Rochet (1985)). In this sense, we obtain a generalization of their result.

This result is intuitive. Recall that the smaller (larger) the magnitude of the elasticity of substitution, the less (more) likely to substitute between $t_{1}$ and $t_{2}$. In the case of the CES function (4.9), the elasticity of substitution is given as $\frac{1}{1-\rho}$ and so it becomes less than a half when $\rho<-1$. Thus, given the total amount of signaling cost, the sender will invest in the attribute in which she is relative superior rather than in the other one.

Consider the job market model as an example. Let $t_{1}$ and $t_{2}$ denote the degrees of how smart a job seeker is and how sociable he is, respectively. The corresponding attributes $s_{1}$ and $s_{2}$ in signal are the GPA and the extracurricular activity, respectively. When $t_{1}$ and $t_{2}$ 
do not substitute each other in determining the job seeker's productivity $\alpha$ and so the wage, a more sociable job seeker will invest his resource in widening his social network rather than in preparing for exams to earn high scores. The reason is that while the latter costs him more, high scores are less likely to substitute his social activity in the wage determination.

The following corollary gives the system of equations that an SE strategy satisfies.

Corollary 4.4.1. Assume that the cost function $c$ is twice continuously differentiable. Suppose the mapping $\sigma: T \rightarrow S$ is an SE strategy in $\mathbf{G}^{n, m}$. Then, for all $t \in T$, there exists a scalar $\mu$ such that

$$
F(\sigma(t), t)=0 \text { and } \nabla_{t} F(\sigma(t), t)=\mu \nabla \alpha(t) .
$$

Here, $\nabla_{t}$ denotes $\left(\partial / \partial_{t_{1}}, \ldots, \partial / \partial_{t_{n}}\right)$.

Proof. Fix $t \in T$. The first equation is simply C2 in Theorem 4.4.1.

To obtain the second one, observe that for all $t^{\prime}$ with $t^{\prime} \sim_{\alpha} t$,

$$
\begin{aligned}
F\left(\sigma(t), t^{\prime}\right) & \geq 0 \\
& =F(\sigma(t), t) .
\end{aligned}
$$

This implies that

$$
t \in \arg \min _{\left\{t^{\prime}: \alpha\left(t^{\prime}\right)=\alpha(t)\right\}} F\left(\sigma(t), t^{\prime}\right) .
$$

Denoting by $\mu$ the multiplier of the minimization problem above, we write the Lagrangian $L$ of the problem:

$$
L\left(t^{\prime}, \mu\right)=F\left(\sigma(t), t^{\prime}\right)+\mu\left(\alpha\left(t^{\prime}\right)-\alpha(t)\right) .
$$

The first order condition $\left.\frac{\partial L\left(t^{\prime}, \mu\right)}{\partial t^{\prime}}\right|_{t^{\prime}=t}=0$ gives the second equation.

This corollary is in particular useful when $n \leq m(\operatorname{or} \operatorname{dim} S \leq \operatorname{dim} T)$, because it allows to directly obtain the candidate $\sigma(t) \in S=\mathbb{R}_{+}^{n}$ of an SE strategy without constructing the set $U_{t}$ and solving the optimization problem in C3. That is, since the system (4.12) consists of $(m+1)$ equations, it determines $\sigma(t) \in \mathbb{R}^{n}$ when $n \leq m$. For example, $\sigma(t)$ in (4.11) in Example 4.4.1 
can be also derived by solving for $s$ the following system of three equations

$$
F(s, t)=\frac{s_{1}}{t_{2}}+\frac{s_{2}}{t_{2}}-\frac{1}{2}\left(\frac{1}{2} t_{1}^{\rho}+\frac{1}{2} t_{2}^{\rho}\right)^{1 / \rho}=0
$$

and

$$
\begin{aligned}
& \frac{\partial F}{\partial t_{1}}(s, t)-\mu \frac{\partial \alpha}{\partial t_{1}}(t)=-\frac{s_{1}}{t_{1}^{2}}-\frac{t_{1}^{\rho-1}}{4}\left(\frac{1}{2} t_{1}^{\rho}+\frac{1}{2} t_{2}^{\rho}\right)^{1 / \rho-1}-\mu \frac{t_{1}^{\rho-1}}{2}\left(\frac{1}{2} t_{1}^{\rho}+\frac{1}{2} t_{2}^{\rho}\right)^{1 / \rho-1}=0 \\
& \frac{\partial F}{\partial t_{2}}(s, t)-\mu \frac{\partial \alpha}{\partial t_{2}}(t)=-\frac{s_{2}}{t_{2}^{2}}-\frac{t_{2}^{\rho-1}}{4}\left(\frac{1}{2} t_{1}^{\rho}+\frac{1}{2} t_{2}^{\rho}\right)^{1 / \rho-1}-\mu \frac{t_{2}^{\rho-1}}{2}\left(\frac{1}{2} t_{1}^{\rho}+\frac{1}{2} t_{2}^{\rho}\right)^{1 / \rho-1}=0 .
\end{aligned}
$$

\subsection{Discussion}

Quinzii and Rochet (1985) consider a multidimensional signaling game with $T=\mathbb{R}_{++}^{n}, S=\mathbb{R}_{+}^{n}$ and $A=\mathbf{R}_{+}$. They assume that the function $\beta: T \rightarrow A$ defined by

$$
\beta(x)=\alpha\left(1 / x_{1}, \ldots, 1 / x_{n}\right)
$$

is convex and the cost function has the form of

$$
c(s, t)=\sum_{i=1}^{n} \frac{s_{i}}{t_{i}} .
$$

Quinzii and Rochet elegantly show that there is a one-to-one correspondence between separating equilibria and solutions to a certain partial differential equation (Theorem 1 in Quinzii and Rochet (1985)). In particular, they also show (Proposition 1 in Quinzii and Rochet (1985)) that there exists a separating strategy $\sigma$ and the type $t$ 's payoff in signaling equilibrium, $U(t):=\alpha(t)-c(\sigma(t), t)$, is given as

$$
U(t)=\int_{0}^{1} \alpha(y t) d y .
$$

Using our results, we will derive their results without solving a partial differential equation.

Assume that $\sigma(t)=\left(\sigma_{1}(t), \ldots, \sigma_{n}(t)\right)$ is a separating strategy.

It is readily verified that the cost function (4.13) satisfies Assumptions 4.3.3, 4.3.4 and 4.3.6 with $g(\lambda)=1 / \lambda$. 
The type $t^{\prime}$ s payoff in separating equilibrium is given as

$$
\begin{aligned}
U(t) & =\alpha(t)-c(\sigma(t), t) \\
& =\alpha(t)-\int_{0}^{1} y t \cdot \nabla \alpha(y t) d y \\
& =\left.y \alpha(y t)\right|_{y=0} ^{1}-\int_{0}^{1} y t \cdot \nabla \alpha(y t) d y \\
& =\int_{0}^{1} \alpha(y t) d y
\end{aligned}
$$

(by integration-by-parts)

This is exactly the same as (4.14).

Next we derive $\sigma$. It follows from (IC) that $U(t)=\max _{t^{\prime} \in T}\left\{\alpha\left(t^{\prime}\right)-\sum_{i=1}^{n} \frac{\sigma_{i}\left(t^{\prime}\right)}{t_{i}}\right\}$. Since $U$ is differentiable as seen in (4.14) and its derivative is $\nabla U(t)=$ $\int_{0}^{1} y \nabla \alpha(y t) d y$, it holds by the envelope theorem

$$
\int_{0}^{1} y \nabla \alpha(y t) d y=\left(\frac{\sigma_{1}(t)}{t_{1}^{2}}, \ldots, \frac{\sigma_{n}(t)}{t_{n}^{2}}\right) .
$$

Thus, we obtain

$$
\sigma_{i}(t)=t_{i}^{2} \int_{0}^{1} y \alpha_{i}(y t) d y \text { for } i=1, \ldots, n,
$$

where $\alpha_{i}$ denotes the partial derivative of $\alpha$ with respect to $i^{\prime}$ th variable $(i=1, \ldots, n)$.

We show that $\lim _{\|t \mid\| \rightarrow 0} \sigma(t)=0$ and (IC) holds and thus $\sigma$ is indeed a separating strategy.

The first condition comes from the following observation: for all $i=$ $1, \ldots, n$,

$$
\begin{aligned}
& \sigma_{i}(t)=t_{i} \int_{0}^{1} y t_{i} \alpha_{i}(y t) d y \\
& \leq t_{i} \sum_{j=1}^{n} \int_{0}^{1} y t_{j} \alpha_{j}(y t) d y \quad \text { (because } \alpha_{j}>0 \text { for all } j \text { ) } \\
& =t_{i} \int_{0}^{1} y t \cdot \nabla \alpha(y t) d y \\
& =t_{i}\left(\alpha(t)-\int_{0}^{1} \alpha(y t) d y\right) \quad \text { (by integration-by-parts) } \\
& \leq t_{i} \alpha(t) \\
& \rightarrow 0 \\
& (\text { as } t \rightarrow \mathbf{0})
\end{aligned}
$$


Before verifying (IC), we prove the following lemma.

Lemma 4.5.1. The mapping $V: \mathbb{R}_{++}^{n} \rightarrow \mathbb{R}$ defined as $V(x)=U(t)$ is convex where $x=\left(x_{1}, \ldots, x_{n}\right)=\left(1 / t_{1}, \ldots, 1 / t_{n}\right)$. And, it is differentiable and its differential is $\nabla V(x)=-\sigma(t)$.

Proof. Notice that

$$
V(x)=\int_{0}^{1} \alpha\left(\frac{y}{x_{1}}, \ldots, \frac{y}{x_{n}}\right) d y=\int_{0}^{1} \beta\left(\frac{x}{y}\right) d y .
$$

Thus, for any $\lambda \in[0,1]$ and $x, x^{\prime} \in \mathbb{R}_{++}^{2}$

$$
\begin{aligned}
V\left(\lambda x+(1-\lambda) x^{\prime}\right) & =\int_{0}^{1} \beta\left(\frac{\lambda x+(1-\lambda) x^{\prime}}{y}\right) d y \\
& \left.\leq \int_{0}^{1}\left\{\lambda \beta\left(\frac{x}{y}\right)+(1-\lambda) \beta\left(\frac{x^{\prime}}{y}\right)\right\} d y \quad \text { (by convexity of } \beta\right) \\
& =\lambda V(x)+(1-\lambda) V\left(x^{\prime}\right)
\end{aligned}
$$

And, it follows from the chain rule that $\nabla V(x)=-\sigma(t)$.

We claim that (IC) holds. For all $t, t^{\prime} \in T$, denoting $x=\left(1 / t_{1}, \ldots, 1 / t_{n}\right)$ and $x^{\prime}=\left(1 / t_{1}^{\prime}, \ldots, 1 / t_{n}^{\prime}\right)$, we have

$$
\begin{aligned}
\alpha\left(t^{\prime}\right)-c\left(\sigma\left(t^{\prime}\right), t^{\prime}\right) & =\alpha\left(t^{\prime}\right)-\sum_{i=1}^{n} \frac{\sigma_{i}\left(t^{\prime}\right)}{t_{i}^{\prime}} \\
& =U\left(t^{\prime}\right) \\
& =V\left(x^{\prime}\right) \\
& \geq V(x)-\sigma(t) \cdot\left(x^{\prime}-x\right) \quad \quad \text { by L } \\
& =U(t)-\sum_{i=1}^{n} \sigma_{i}(t) \cdot\left(\frac{1}{t_{i}^{\prime}}-\frac{1}{t_{i}}\right) \\
& =\alpha(t)-\sum_{i=1}^{n} \frac{\sigma_{i}(t)}{t_{i}}-\sum_{i=1}^{n} \sigma_{i}(t) \cdot\left(\frac{1}{t_{i}^{\prime}}-\frac{1}{t_{i}}\right) \\
& =\alpha(t)-\sum_{i=1}^{n} \frac{\sigma_{i}(t)}{t_{i}^{\prime}} \\
& =\alpha(t)-c\left(\sigma\left(t^{\prime}\right), t\right) .
\end{aligned}
$$

Thus, (IC) follows.

Therefore, we conclude that $\sigma$ defined in (4.15) is a separating strategy. 


\subsection{Conclusion}

This chapter presented a characterization of an SE strategy in pure signaling games with multi-dimensional types and signals. The main difficulty in deriving an SE strategy comes from the fact that the available incentive scheme is one-dimensional monetary payment while the type is multi-dimensional. To overcome this difficulty, we used the known results in single dimensional games and the ideas from traditional consumer theory. We first considered a family of single dimensional parts of the original game and drew information about the signaling cost in SE of the original game. Based on this information, we could determine the signals in SE. To this end, we used the same idea as the derivation of the Hicksian demand function from the expenditure function. Lastly, as one example of applications of our results, we reconstructed some of the pioneering results by Quinzii and Rochet (1985).

The limit of our results is the restriction on the form of signaling cost functions. It would be a potentially interesting future research avenue to consider more general forms of signaling cost functions. 


\section{Chapter 5}

\section{Voucher Privatization}

\subsection{Introduction}

Voucher privatization was used to privatize enterprises in Russia in the early 1990s. The privatization gave away a significant portion of stateowned assets to the general population. This transfer of assets was unprecedented in the sense that it was fast and comprehensive.

The procedure of voucher privatization used in Russia is as follows. In the beginning, every citizen, including minors, received a unit of voucher with a given face value $(10,000$ rubles). Vouchers were freely traded on organized exchanges throughout the country. Then, an auction was held, where any person interested in purchasing shares of a particular enterprise could submit a bid in terms of these vouchers. Each bidder received the number of shares inversely proportional to the number of vouchers tendered (the proportional-share allocation rule). For example, if 1,000 shares of a certain enterprise were auctioned and 40 vouchers were submitted, then each voucher holder received 25 shares per voucher submitted.

The reason that the Russian government chose this seemingly arbitrary privatization scheme at that time was mainly to gain public support for the reformist politicians (Boycko et al. (1993)). Voucher privatization fit this mandate particularly well because it was perceived by the general population as an economic reform that can unambiguously benefit them. In 
addition, the political support for privatization were expected to spill over to other reforms and lead to create supporters for reform in the population and reduce the power of its opponents.

Given that the use of voucher privatization over traditional auctions was motivated by political rather than economic considerations, two questions naturally arise. First, has economic efficiency fallen victim to political feasibility? Second, has voucher privatization sacrificed revenue potential? In this chapter, we compare the outcome of voucher privatization with that of direct privatization by a government. To address the first question, we model a strategic interaction between a potential raider and voucher holders occurring in voucher privatization as a tender offer game and show that its outcome is inefficient. Regarding the second, we show that the government's revenue under direct privatization is strictly higher than the total revenue of the general public under voucher privatization and thus it would be better for the public when a government sells directly the firm and redistributes the proceeds to them.

There are two strands of research related to this chapter. One strand is on voucher privatization. Most existing literature on voucher privatization focuses on the scheme's historical and political background and the process' impact on transition economies. Excellent studies on privatization in Russia include Boycko et al. (1993) and Boycko et al. (1994). Yusupova (2006) contains an extensive survey of this literature and also deals with a model of voucher auctions from the perspective of an investment portfolio decision. However, there has been very few theoretical work to analyze it and little is known about its outcome or efficiency and distribution properties. An exception is Katz and Owen (1997) who analyze equilibrium bidding in a model similar to Boycko et al. (1994)'s description under the assumption of perfect information.

The other strand is the literature on tender offers. Tender offers have been studied a lot with particular emphasis on the problems associated with the free-rider problem in takeover bidding. Grossman and Hart (1980) and Shleifer et al. (1986) offer an analysis of asymmetric information in tender offer games. Both papers focus exclusively on pooling equilibria. Hirshleifer and Titman (1990) and Chowdhry and Jegadeesh (1994) construct separating equilibria in a tender offer game. These equilibria 
require shareholders to randomize their decision such that bids at lower prices fail with higher probability. Marquez and Yilmaz (2008) reverse the information asymmetry and study a tender offer game in which target shareholders receive private information signals so that bidders face a winner's curse problem as opposed to a signaling problem.

The rest of the chapter is organized as follows. Section 5.2 presents the model of voucher privatization. Section 5.3 analyzes an equilibrium of the model. In Section 5.4, we consider the alternative case of direct privatization in which a government sells the firm directly and compare the outcomes of voucher privatization and direct privatization. Section 5.5 concludes.

\subsection{Model}

There is a firm which is currently owned and run by the government $(G)$. A potential raider $(R)$ wishes to buy and run the firm. Citizens are represented by the unit interval $[0,1]$, each $i \in[0,1]$ indicating an individual citizen. There is a measure 1 continuum of citizens, each of whom is indexed by $i$. The fraction of shares needed to gain control over the firm is at least $q \in[0.5,1]$. We assume that all players are risk neutral. We will use "he" and "she" to denote $R$ and a citizen, respectively.

The value $v$ of the firm is assumed to be equal to its owner's management ability. On the one hand, $G$ 's management ability is summarized by $t_{G}>0$, which is common knowledge. On the other hand, $R^{\prime}$ s management ability is summarized by $t \in\left\{t_{L}, t_{H}\right\}$, or his type, with $0<t_{L} \leq t_{G}<t_{H}$. Let $\mu \in(0,1)$ be the probability that $t=t_{H}$ and assume that $\mu$ is common knowledge. In addition, there is private benefit $B \in\left[0, t_{H}-t_{L}\right]$ of being the manager of the firm. Hence, though $R$ may destroy the firm's value when running the firm, he may wish to take over partly due to the private benefit.

For $k, l \in\{G, R\} \cup[0,1]$, we denote by $m_{l k}$ the net money transfer from player $l$ to $k$ and by $s_{k} \in[0,1]$ the fraction of shares of the firm that player $k$ has. Player $k^{\prime}$ s payoff is then

$$
m_{G k}+m_{R k}+\int_{0}^{1} m_{i k} d i+s_{k} v+B \cdot 1_{\left\{s_{k} \geq q\right\}},
$$


where $v \in\left\{t_{L}, t_{G}, t_{H}\right\}$.

The possible outcome in voucher privatization is that the firm is under the management of either $G$ or $R$, i.e., $s_{G} \geq q$ or $s_{R} \geq q$. In addition, $m_{l k}+$ $m_{k l}=0$ and $m_{l l}=0$ for $k, l \in\{G, R\} \cup[0,1]$ and the sum of all fractions of shares is unity. Hence, the sum of all payoffs in society becomes

$$
v+B
$$

Therefore, efficiency will be achieved if and only if the $t_{H}$-type $R$ gains control over the firm and the $t_{L}$-type does not.

\subsubsection{Timeline}

The procedure of voucher privatization is as follows. In the beginning, every citizen receives one voucher. Vouchers are allowed to be freely traded on an organized exchange, which we call a voucher market. After the voucher market closes, an auction for shares of the firm is held, where any person interested in purchasing shares can submit a bid in terms of vouchers she possesses. Each bidder receives the number of shares inversely proportional to the number of vouchers tendered. Since a voucher itself has no value after the auction, every voucher will be tendered during the auction. This implies that one unit of voucher is eventually equivalent to one unit of share of the firm.

As such, $G$ plays the simple role to issue vouchers and distribute them to the citizens. Thus our model of voucher privatization is primarily concerned with the strategic interaction in the voucher market between $R$ and a group of citizens. From now on, I interchangeably use two terms voucher holders and citizens. The timeline of the game is as follows:

1. Nature chooses $R^{\prime}$ s type $t$. The realized type is revealed only to $R$. Voucher holders do not know $R^{\prime}$ 's type and instead receive a signal $s \in[0,1]$ independently drawn from an identical distribution $F(s \mid t)$ with density $f(s \mid t)$, which depends on $R^{\prime}$ s type $t$.

2. $R$ makes a tender offer at price $p$ per voucher in the voucher market. Upon observing $p$, voucher holders simultaneously decide whether to tender their vouchers or keep them. 
3. If more than $q$ vouchers are tendered, $R$ gains control over the firm. Otherwise, $G$ keeps holding control over the firm.

\subsubsection{Information structure}

Voucher holders share the prior belief that the probability that $R$ is of $t_{H^{-}}$ type is $\mu$. The posterior belief conditional on $s$ is

$$
\mathbb{P}\left(t_{H} \mid s\right)=\frac{\mu f\left(s \mid t_{H}\right)}{\mu f\left(s \mid t_{H}\right)+(1-\mu) f\left(s \mid t_{L}\right)},
$$

where $\mathbb{P}(. \mid$.) denotes a conditional probability.

We make the following assumptions on the posterior belief.

Assumption 5.2.1. $\mathbb{P}\left(t_{H} \mid s\right)$ is continuous in $s$.

Assumption 5.2.2. $\mathbb{P}\left(t_{H} \mid s\right)$ is strictly increasing in s.

Assumption 5.2.3. $\mathbb{P}\left(t_{H} \mid 0\right)=0$ and $\mathbb{P}\left(t_{H} \mid 1\right)=1$.

Assumption 5.2.2 means that the higher $s$, for instance, the higher possibility of $t=t_{H}$. A sufficient condition for this assumption is that the strict monotone likelihood ratio property (MLRP) holds, i.e., $\frac{f\left(s \mid t_{H}\right)}{f\left(s \mid t_{L}\right)}$ is strictly increasing in $s$. One immediate implication is that the expected type of $R$ conditional on $s, \mathbb{P}\left(t_{H} \mid s\right) \cdot t_{H}+\mathbb{P}\left(t_{L} \mid s\right) \cdot t_{L}=\mathbb{P}\left(t_{H} \mid s\right) \cdot\left(t_{H}-t_{L}\right)+t_{L}$, is strictly increasing in $s$.

Assumption 5.2.3 implies that the lowest (highest) signal indicates $t=$ $t_{L}\left(t=t_{H}\right.$, respectively) for sure. A sufficient condition for this assumption is that $f\left(0 \mid t_{H}\right)=f\left(1 \mid t_{L}\right)=0$ and $f\left(0 \mid t_{L}\right) \neq 0, f\left(1 \mid t_{H}\right) \neq 0$.

\subsubsection{Payoffs and Equilibrium}

To describe the payoffs of $R$ and voucher holders, let

$$
a_{i}= \begin{cases}1 & \text { if voucher holder } i \text { tenders } \\ 0 & \text { if voucher holder } i \text { does not tender }\end{cases}
$$

and

$$
\alpha=\int_{0}^{1} a_{i} d i
$$




\begin{tabular}{|c|c|c|}
\hline & $\alpha \geq q$ & $\alpha<q$ \\
\hline$a_{i}=1$ & $p$ & $p$ \\
\hline$a_{i}=0$ & $t$ & $t_{G}$ \\
\hline
\end{tabular}

Table 5.1: Voucher holder's payoff

which is the proportion of voucher holders who tender their vouchers. Here, we assume that the set of voucher holders who tender their vouchers is measurable.

The payoff of voucher holder $i$ is

$$
u_{i}\left(p, a_{i}, \alpha, t\right)=\left\{\begin{array}{cl}
p & \text { if } a_{i}=1 \\
t & \text { if } a_{i}=0 \text { and } \alpha \geq q \\
t_{G} & \text { if } a_{i}=0 \text { and } \alpha<q
\end{array}\right.
$$

which is summarized in Table 5.1.

$R$ 's payoff consists of two components when $R$ succeeds in gaining control over the firm. One is the profit of the vouchers that he purchases, $\alpha(t-p)$, and the other is the private benefit of being a manager, $B$. When he fails to gain control, $G$ continues to manage the firm and so the firm's value stays at $t_{G}$. Thus, $R^{\prime}$ s payoff is equal to

$$
u_{R}(p, \alpha, t)= \begin{cases}\alpha(t-p)+B & \text { if } \alpha \geq q \\ \alpha\left(t_{G}-p\right) & \text { otherwise }\end{cases}
$$

An equilibrium for the game consists of strategies for $R$ and for voucher holders such that no player has an incentive to deviate. We restrict our attention on pure strategies.

$R^{\prime}$ 's strategy is a pair of real numbers, $\left(p_{L}, p_{H}\right)$, where $p_{L}$ and $p_{H}$ are the prices which $t_{L}$-type and $t_{H}$-type will offer, respectively. Notice that offering any price above $t_{H}$ is not optimal for $R$. The reason is that a voucher holder will choose to tender at any price $p>t_{H}$ since she can get at best $t_{H}$ when she does not tender. On the other hand, accepting $p<t_{L}$ is not optimal for voucher holders since she can get at least $t_{L}$ when she does not tender. Thus we can put the restriction $p \in\left[t_{L}, t_{H}\right]$ without loss of generality. 
Following $R^{\prime}$ s tender offer $p$, each voucher holder, based on the observed signal, decides whether or not to tender her voucher in the voucher market. Hence, voucher holder $i^{\prime}$ s strategy $a_{i}$ is a measurable mapping from $[0,1] \times\left[t_{L}, t_{H}\right]$ to $\{0,1\}$. We only consider a symmetric strategy $a$, that is, $a_{i}=a_{j}=a$ for all $i, j$.

When all voucher holders play $a$, the proportion of voucher holders tendering their vouchers is equal to

$$
\alpha(a, p, t)=\int_{0}^{1} a d i=\mathbb{P}(\{s \mid a(s, p)=1\} \mid t) .
$$

where $p$ is the offered price and $t$ is $R^{\prime}$ s type.

Hence, we can write $R^{\prime}$ s payoff as

$$
u_{R}(p, \mathbb{P}(\{s \mid a(s, p)=1\} \mid t), t) .
$$

As for voucher holders, notice that a single voucher holder cannot influence the outcome and thus the expression (5.4) does not depend on a single voucher holder's choice. Thus, the payoff of voucher holder $i$ when all voucher holders other than $i$ use $a$ is

$$
u_{i}\left(p, a_{i}, \mathbb{P}(\{s \mid a(s, p)=1\} \mid t), t\right)
$$

Definition 5.2.1. A strategy profile $\left(\left(p_{L}, p_{H}\right), a\right)$ of strategies is an equilibrium if

1. For $j=H, L$

$$
p_{j}=\arg \max _{p \in\left[t_{L}, t_{H}\right]} u_{R}\left(p, \mathbb{P}\left(\{s \mid a(s, p)=1\} \mid t_{j}\right), t_{j}\right) .
$$

2. For each $i$ and $\left(s_{i}, p\right) \in[0,1] \times\left\{p_{L}, p_{H}\right\}$,

$$
a\left(s_{i}, p\right)=\arg \max _{a_{i} \in\{0,1\}} \sum_{j \in\{H, L\}} u_{i}\left(p, a_{i}, \mathbb{P}\left(\{s \mid a(s, p)=1\} \mid t_{j}\right), t_{j}\right) \cdot \mathbb{P}\left(t_{j} \mid p, s_{i}\right)
$$

where for $j=H, L$

$$
\mathbb{P}\left(t_{j} \mid p, s_{i}\right)=\left\{\begin{array}{cl}
\mathbb{P}\left(t_{j} \mid s_{i}\right) & \text { if } p=p_{L}=p_{H} \\
1 & \text { if } p_{L} \neq p_{H} \text { and } p=p_{j}
\end{array}\right.
$$


If $p_{H}=p_{L}$, we call it a pooling equilibrium. If $p_{H} \neq p_{L}$, we call it a separating equilibrium.

Condition (5.7) states that each type of $R$ responds optimally to voucher holders' strategy $a$. Condition (5.8) states a voucher holder chooses an action that maximizes her expected payoff, where the expectation is taken with respect to the distribution $\mathbb{P}\left(. \mid p, s_{i}\right)$ following $p$ and $s_{i}$. Condition (5.9) requires that $\mathbb{P}\left(. \mid p, s_{i}\right)$ be determined by Bayesian updating whenever possible.

\subsection{Analysis}

We find an equilibrium in the game defined above. We examine the property of separating equilibrium and then turn focus on pooling equilibrium. We assume that if a voucher holder is indifferent between tendering and not tendering, she will choose to tender her voucher to $R$. This may be justified if it is costly to keep a voucher and participate in the voucher auction later.

\subsubsection{Separating equilibrium}

The following lemma is useful to characterize the outcomes in separating equilibrium.

Lemma 5.3.1. Suppose $\left(\left(p_{L}, p_{H}\right), a\right)$ is a separating equilibrium. Then, $a(s, p)=$ $a\left(s^{\prime}, p\right)$ for all $s, s^{\prime} \in[0,1]$ and $p \in\left\{p_{H}, p_{L}\right\}$.

Proof. When $p=p_{k}(k=H, L)$, by (5.9) the objective function in (5.8) becomes

$$
u_{i}\left(p_{k}, a_{i}, \mathbb{P}\left(\left\{s \mid a\left(s, p_{k}\right)=1\right\} \mid t_{k}\right), t_{k}\right) .
$$

This function does not depend on $s$. Thus, its maximizer $a(s, p)$ does not depend on $s$ as well.

The meaning of the lemma above is that in separating equilibrium a voucher holder does not base her decision on the signal because an offered price fully reveals $R^{\prime}$ s type. 
Theorem 5.3.1. $\quad$ 1. The strategy profile $\left(\left(\bar{p}_{L}, \bar{p}_{H}\right), \bar{a}\right)$ is a separating equilibrium where $\bar{p}_{L}, \bar{p}_{H} \in\left[t_{L}, t_{G}\right), \bar{p}_{L} \neq \bar{p}_{H}$ and $\bar{a}(s, p)=0$ for all $s, p$. The equilibrium payoffs of voucher holders and $R$ are $t_{G}$ and zero.

2. The strategy profile $\left(\left(\tilde{p}_{L}, \tilde{p}_{H}\right), \tilde{a}\right)$ is a separating equilibrium where $\tilde{p}_{L} \in$ $\left[t_{L}, t_{G}\right), \tilde{p}_{H}=t_{G}$ and

$$
\tilde{a}(s, p)= \begin{cases}1 & \text { if } p=\tilde{p}_{H} \\ 0 & \text { otherwise }\end{cases}
$$

The equilibrium expected payoffs of voucher holders and $R$ are $\mu t_{H}+(1-$ $\mu) t_{G}$ and $\mu B$, respectively.

3. The outcome in separating equilibrium is either no trades occurs in the voucher market or only the $t_{H}$-type $R$ succeeds in gaining control over the firm.

Proof. 1. It suffices to show that conditions 1 and 2 in Definition 5.2.1 are respected.

We first check condition 1 . Suppose all voucher holders play $\bar{a}$. Then, for $j=H, L$ and all $p \in\left[t_{L}, t_{H}\right]$

$$
u_{R}\left(p, \mathbb{P}\left(\{s \mid \bar{a}(s, p)=1\} \mid t_{j}\right), t_{j}\right)=u_{R}\left(p, 0, t_{j}\right)=0,
$$

that is, $R$ always gets zero payoff regardless of his choice of $p$. Hence, $\left(\bar{p}_{L}, \bar{p}_{H}\right)$ satisfies condition 1 .

To verify condition 2 , fix voucher holder $i$. Suppose that $R$ plays $\left(\bar{p}_{L}, \bar{p}_{H}\right)$ and all voucher holder other than $i$ use $\bar{a}$. We show that it is optimal for $i$ to play $\bar{a}$. Notice that since condition 2 is concerned only with the cases $p=\bar{p}_{j}(j=H, L)$, we do not need to consider other cases.

When $\bar{p}_{L}$ is offered, regardless of $i$ 's choice

$$
\mathbb{P}\left(\{s \mid \bar{a}(s, p)=1\} \mid t_{j}\right)=0(j=H, L),
$$

and so the firm continues to be under $G^{\prime}$ s management and its value remains $t_{G}$. If voucher holder $i$ tenders his voucher $\left(a_{i}=1\right)$, his payoff is $\bar{p}_{L}$. Otherwise $\left(a_{i}=0\right)$, his payoff is $t_{G}$. Since $\bar{p}_{L}<t_{G}, a_{i}=0$ is optimal.

When $\bar{p}_{H}$ is offered, for the same reason, the firm's value remains $t_{G}$ regardless of $i$ 's choice. If voucher holder $i$ sells his voucher $\left(a_{i}=1\right)$, his 
payoff is $\bar{p}_{H}$. Otherwise $\left(a_{i}=0\right)$, his payoff is $t_{G}$. Since $\bar{p}_{H}<t_{G}, a_{i}=0$ is optimal again.

Therefore, it is optimal for voucher holder $i$ to play $\bar{a}$.

As for the payoffs, since no trade occurs, $G$ continues to run the firm. Thus, $R^{\prime}$ s payoff is zero and each voucher holder gets the payoff of $t_{G}$.

2. We first check condition 1. Suppose all voucher holders play $\tilde{a}$. Then, for $j=H, L$

$$
\mathbb{P}\left(\left\{s \mid \tilde{a}\left(s, \tilde{p}_{H}\right)=1\right\} \mid t_{j}\right)=1 \quad \text { and } \quad \mathbb{P}\left(\{s \mid \tilde{a}(s, p)=1\} \mid t_{j}\right)=0 \text { for all } p \neq \tilde{p}_{H} .
$$

Thus, the $t_{H}$-type $R$ will get $1 \cdot\left(t_{H}-t_{H}\right)+B=B$ if he offers $\tilde{p}_{H}$. If he offers other prices, his payoff will be zero. Since $B>0$, it is optimal for $t_{H}$-type to offer $\tilde{p}_{H}$.

As for the $t_{L}$-type $R$, he will get $1 \cdot\left(t_{L}-t_{H}\right)+B$ if he offers $\tilde{p}_{H}$. Offering other prices will give him zero. Since it is assumed that $B \leq t_{H}-t_{L}$, it is optimal for $t_{L}$-type to offer $\tilde{p}_{L}$

Hence, $\left(\tilde{p}_{L}, \tilde{p}_{H}\right)$ satisfies condition 1 .

To verify condition 2 , fix voucher holder $i$. Suppose that $R$ plays $\left(\tilde{p}_{L}, \tilde{p}_{H}\right)$ and all voucher holder other than $i$ use $\tilde{a}$.

When $\tilde{p}_{L}$ is offered, regardless of $i$ 's choice

$$
\mathbb{P}\left(\left\{s\left|\tilde{a}\left(s, \tilde{p}_{L}\right)=1\right| t_{j}\right\}\right)=0(j=H, L)
$$

and so the firm continues to be under $G^{\prime}$ 's management and its value remains $t_{G}$. If voucher holder $i$ tenders his voucher $\left(a_{i}=1\right)$, his payoff is $\tilde{p}_{L}$. Otherwise $\left(a_{i}=0\right)$, his payoff is $t_{G}$. Since $\tilde{p}_{L}<t_{G}, a_{i}=0$ is optimal.

When $\tilde{p}_{H}$ is offered, $\mathbb{P}\left(\left\{s\left|\tilde{a}\left(s, \tilde{p}_{H}\right)=1\right| t_{j}\right\}\right)=1(j=H, L)$ and the firm will be under $R^{\prime}$ s management. Because $R$ is of $t_{H}$-type, the firm's value will increase to $t_{H}$. If voucher holder $i$ tenders his voucher $\left(a_{i}=1\right)$, his payoff is $\tilde{p}_{H}=t_{H}$. Otherwise $\left(a_{i}=0\right)$, his payoff is $t_{H}$. Since we assume that this indifference is resolved in favor of tendering, $a_{i}=1$ is optimal.

Therefore, it is optimal for voucher holder $i$ to play $\tilde{a}$.

To determine the equilibrium payoffs, note that if he is of $t_{H}$-type, $R$ runs the firm and, otherwise, $G$ keeps running the firm. Thus, $R^{\prime}$ s expected payoff is $\mu B$ and the payoff of each voucher holder is $\mu t_{H}+(1-\mu) t_{G}$. 
3. If the strategy $a$ of voucher holders is part of a separating equilibrium, $a\left(s, p_{j}\right)(j=H, L)$ does not depend on $s$ by Lemma 5.3.1 and it is either 0 or 1 .

The equilibrium in the first claim, in which $\left(\bar{a}\left(s, p_{L}\right), \bar{a}\left(s, p_{H}\right)\right)=(0,0)$, results in no trade in voucher market. In the equilibrium in the second claim, $\left(\tilde{a}\left(s, p_{L}\right), \tilde{a}\left(s, p_{H}\right)\right)=(0,1)$ and only the $t_{H}$-type gains control over the firm. Hence, it suffices to show that in separating equilibrium, neither $\left(a\left(s, p_{L}\right), a\left(s, p_{H}\right)\right)=(1,0)$ or $(1,1)$ is possible.

Suppose there is a separating equilibrium with $\left(a\left(s, p_{L}\right), a\left(s, p_{H}\right)\right)=(1,1)$. Then, both types succeed in gaining control. But, if $p_{H}>p_{L}$, it is profitable for the $t_{H}$-type $R$ to offer the lower price $p_{L}$ instead of $p_{H}$ because by doing so, he can still gain control while paying strictly less money to voucher holders. Otherwise, the $t_{L}$-type $R$ will offer the lower price $p_{H}$ instead of $p_{L}$ for the same reason. Hence, this cannot be an equilibrium.

Suppose there is a separating equilibrium with $\left(a\left(s, p_{L}\right), a\left(s, p_{H}\right)\right)=(1,0)$. Then, for $j=H, L$

$$
\mathbb{P}\left(\left\{s \mid a\left(s, p_{L}\right)=1\right\} \mid t_{j}\right)=1 \quad \text { and } \quad \mathbb{P}\left(\left\{s \mid a\left(s, p_{H}\right)=1\right\} \mid t_{j}\right)=0
$$

Hence, the payoff to the $t_{j}$-type $R$ is

$$
u_{R}\left(p, \mathbb{P}\left(\{s \mid a(s, p)=1\} \mid t_{j}\right), t_{j}\right)=\left\{\begin{array}{cl}
0 & \text { if } p=p_{H} \\
\left(t_{j}-p_{L}\right)+B & \text { if } p=p_{L}
\end{array}\right.
$$

Condition 1 in Definition 5.2.1 puts the constraint that

$$
\begin{gathered}
u_{R}\left(p_{H}, \mathbb{P}\left(\left\{s \mid a\left(s, p_{H}\right)=1\right\} \mid t_{H}\right), t_{H}\right) \geq u_{R}\left(p_{L}, \mathbb{P}\left(\left\{s \mid a\left(s, p_{L}\right)=1\right\} \mid t_{H}\right), t_{H}\right) \\
\Longleftrightarrow 0 \geq\left(t_{H}-p_{L}\right)+B
\end{gathered}
$$

and

$$
\begin{gathered}
u_{R}\left(p_{L}, \mathbb{P}\left(\left\{s \mid a\left(s, p_{L}\right)=1\right\} \mid t_{L}\right), t_{L}\right) \geq u_{R}\left(p_{H}, \mathbb{P}\left(\left\{s \mid a\left(s, p_{H}\right)=1\right\} \mid t_{L}\right), t_{L}\right) \\
\Longleftrightarrow\left(t_{L}-p_{L}\right)+B \geq 0,
\end{gathered}
$$

which implies $\left(t_{L}-p_{L}\right)+B \geq\left(t_{H}-p_{L}\right)+B$, or equivalently $t_{L} \geq t_{H}$. This is a contradiction.

The desired result follows. 


\subsubsection{Pooling equilibrium}

We consider a pooling equilibrium. When both types of $R$ offer the same price $p$, it does not reveal any information about $R$ 's type and the belief of voucher holder is equal to (5.1). Thus, a lower signal indicates a higher possibility of $R$ being of $t_{L}$-type and a voucher holder with low signal is more willing to tender her voucher. In this sense, a natural kind of voucher holder's strategy we might consider is one where a voucher holder tenders her voucher only if she observes a signal under some cutoff point $k$, which may depend on $p$ :

$$
a^{k}(s)= \begin{cases}1 & \text { if } s \leq k \\ 0 & \text { otherwise }\end{cases}
$$

We will refer to this strategy $a^{k}$ as the cutoff strategy at $k$. The following lemma shows that voucher holders' strategies indeed have a threshold structure in pooling equilibrium, if it exists.

Before addressing results, it is convenient to define $s(p)$ to be $s$ such that for a given $p \in\left[t_{L}, t_{H}\right]$,

$$
\mathbb{P}\left(t_{H} \mid s\right) \cdot t_{H}+\mathbb{P}\left(t_{L} \mid s\right) \cdot t_{L}=p .
$$

that is, the signal at which the price of the voucher is equal to the expected payoff of the voucher when $R$ manages the firm.

Observe that such $s(p)$ uniquely exists for each $p \in\left[t_{L}, t_{H}\right]$ because the function $s \mapsto \mathbb{P}\left(t_{H} \mid s\right) \cdot t_{H}+\mathbb{P}\left(t_{L} \mid s\right) \cdot t_{L}$ is continuous by Assumption 5.2.1, strictly increasing by Assumption 5.2.2 and its range is $\left[t_{L}, t_{H}\right]$ by Assumption 5.2.3. Note also that the mapping $p \mapsto s(p)$ is strictly increasing.

Lemma 5.3.2. Suppose that $\left(\left(p^{e}, p^{e}\right), a\right)$ is a pooling equilibrium. Then,

(a) both types of $R$ succeed in gaining control over the firm in the equilibrium.

(b) $a\left(s, p^{e}\right)=a^{s\left(p^{e}\right)}(s)$ for all $s$, that is, $a\left(s, p^{e}\right)$ is the cutoff strategy at $s\left(p^{e}\right)$;

(c) $s\left(p^{e}\right) \geq s_{q}^{H}$, where $q \in[0.5,1]$ is the minimum share to gain control over the firm and $s_{q}^{H}$ is the q-quantile of the distribution $F\left(s \mid t_{H}\right)$.

Proof. Let $\left(\left(p^{e}, p^{e}\right), a\right)$ be a pooling equilibrium. We consider the subgame after $p^{e} \in\left[t_{L}, t_{H}\right]$ is offered. 
Fix voucher holder $i$ and suppose that all voucher holders other than $i$ play the strategy $a$.

If voucher holder $i$ tenders her voucher $\left(a_{i}=1\right)$, she will earn $p^{e}$. Otherwise, her expected payoff is

$$
\begin{aligned}
u\left(s_{i}\right) & :=\mathbb{E}\left[t \cdot 1\left\{\alpha\left(a, p^{e}, t\right) \geq q\right\} \mid p^{e}, s_{i}\right] \\
& \left.=\mathbb{E}\left[t \cdot 1\left\{\alpha\left(a, p^{e}, t\right) \geq q\right\} \mid s_{i}\right] \quad \text { (because } \mathbb{P}\left(t \mid p^{e}, s_{i}\right)=\mathbb{P}\left(t \mid s_{i}\right)\right) \\
& =\mathbb{P}\left(t_{H} \mid s_{i}\right) \cdot t_{H} \cdot \mathbb{E}\left[1\left\{\alpha\left(a, p^{e}, t_{H}\right) \geq q\right\}\right]+\mathbb{P}\left(t_{L} \mid s_{i}\right) \cdot t_{L} \cdot \mathbb{E}\left[1\left\{\alpha\left(a, p^{e}, t_{L}\right) \geq q\right\}\right] \\
& =\mathbb{P}\left(t_{H} \mid s_{i}\right) \cdot t_{H} \cdot 1\left\{\alpha\left(a, p^{e}, t_{H}\right) \geq q\right\}+\mathbb{P}\left(t_{L} \mid s_{i}\right) \cdot t_{L} \cdot 1\left\{\alpha\left(a, p^{e}, t_{L}\right) \geq q\right\}
\end{aligned}
$$

(because $\alpha\left(a, p^{e}, t_{j}\right)$ is deterministic)

where $s_{i}$ is the signal she receives.

We need to compare $u\left(s_{i}\right)$ and $p^{e}$ to determine $i^{\prime}$ s behavior. To this end, we prove two claims.

Claim 5.3.1. $\alpha\left(a, p^{e}, t_{H}\right) \geq q$.

Suppose not, i.e., $\alpha\left(a, p^{e}, t_{H}\right)<q$. Then,

$$
u\left(s_{i}\right)=\mathbb{P}\left(t_{L} \mid s_{i}\right) \cdot t_{L} \cdot 1\left\{\alpha\left(a, p^{e}, t_{L}\right) \geq q\right\} \leq t_{L} \leq p^{e}
$$

and thus it is optimal for $i$ to tender her voucher regardless of her signal. But, this holds for all other voucher holders and so all of them will eventually tender their vouchers. This contradicts the assumption $\alpha\left(a, p^{e}, t_{H}\right)<q$. This is a contradiction.

Due to Claim 5.3.1, we write

$$
\begin{aligned}
u\left(s_{i}\right) & =\mathbb{P}\left(t_{H} \mid s_{i}\right) \cdot t_{H}+\mathbb{P}\left(t_{L} \mid s_{i}\right) \cdot t_{L} \cdot 1\left\{\alpha\left(a, p^{e}, t_{L}\right) \geq q\right\} \\
& =\mathbb{P}\left(t_{H} \mid s_{i}\right)\left(t_{H}-t_{L} \cdot 1\left\{\alpha\left(a, p^{e}, t_{L}\right) \geq q\right\}\right)+t_{L} \cdot 1\left\{\alpha\left(a, p^{e}, t_{L}\right) \geq q\right\}
\end{aligned}
$$

which allows us to study the structure of $u\left(s_{i}\right)$.

Claim 5.3.2. The mapping $s_{i} \mapsto u\left(s_{i}\right)$ is continuous, strictly increasing, and $u(0) \leq t_{L}, u(1)=t_{H}$. Hence, there exists $\bar{s}$ such that $s_{i} \leq \bar{s}$ if and only if $u\left(s_{i}\right) \leq$ $p^{e}$.

Continuity follows because $\mathbb{P}\left(t_{H} \mid s_{i}\right)$ is assumed to be continuous in $s_{i}$ (Assumption 5.2.1). In addition, since $t_{H}>t_{L} \geq t_{L} \cdot 1\left\{\alpha\left(a, p^{e}, t_{L}\right) \geq q\right\}$ and 
$s_{i} \mapsto \mathbb{P}\left(t_{H} \mid s_{i}\right)$ is strictly increasing by Assumption 5.2.2, $u\left(s_{i}\right)$ is strictly increasing.

Because $\mathbb{P}\left(t_{H} \mid 1\right)=1$ and $\mathbb{P}\left(t_{H} \mid 0\right)=0$, we have $u(1)=t_{H}$ and $u(0)=t_{L}$. $1\left\{\alpha\left(a, p^{e}, t_{L}\right) \geq q\right\} \leq t_{L}$, as desired.

Lastly, by continuity of $u$, for given $p^{e} \in\left[t_{L}, t_{H}\right]$ there exists $\bar{s}$ such that $p^{e}=u(\bar{s})$. Moreover, by strict monotonicity of $u, s_{i} \leq \bar{s}$ if and only if $u\left(s_{i}\right) \leq$ $p^{e}$. Claim 5.3.2 is proven.

By Claim 5.3.2, voucher holder $i$ will tender her voucher if and only if $s_{i} \leq \bar{s}$ because she will tender her voucher if and only if $u\left(s_{i}\right) \leq p^{e}$. Thus, the optimal strategy for $i$ at $p=p^{e}$ is

$$
a\left(s_{i}, p^{e}\right)= \begin{cases}1 & \text { if } s_{i} \leq \bar{s} \\ 0 & \text { otherwise }\end{cases}
$$

This is true for all $i$. Hence, every voucher holder will use the cutoff strategy around $\bar{s}$.

Now we verify that $\bar{s}=s\left(p^{e}\right)$. To this end, we show the following claim.

Claim 5.3.3. $\bar{s} \geq s_{q}^{H}$ and $\alpha\left(a, p^{e}, t_{L}\right) \geq q$.

The first comes from the following observation:

$$
\begin{aligned}
F\left(\bar{s} \mid t_{H}\right) & =\mathbb{P}\left(s \leq \bar{s} \mid t_{H}\right) \\
& =\mathbb{P}\left(\left\{s \mid a\left(s, p^{e}\right)=1\right\} \mid t_{H}\right) \\
& =\alpha\left(a, p^{e}, t_{H}\right) \\
& \geq q .
\end{aligned}
$$

To see the second one, observe

$$
\begin{aligned}
\alpha\left(a, p^{e}, t_{L}\right) & =\mathbb{P}\left(s \leq \bar{s} \mid t_{L}\right) \\
& =F\left(\bar{s} \mid t_{L}\right) \\
& \geq F\left(s_{q}^{H} \mid t_{L}\right) \\
& \geq F\left(s_{q}^{H} \mid t_{H}\right) \\
& =q .
\end{aligned}
$$

The second inequality is due to the fact that the MLRP condition implies first order stochastic dominance (See, e.g., the Appendix B of Krishna (2002)). Thus, the desired result follows. 
We are ready to prove assertions (a), (b) and (c). Assertion (a) follows from Claims 5.3.1 and 5.3.3.

Claims 5.3.1 and 5.3.3 also implies

$$
u\left(s_{i}\right)=\mathbb{P}\left(t_{H} \mid s_{i}\right) \cdot t_{H}+\mathbb{P}\left(t_{L} \mid s_{i}\right) \cdot t_{L}
$$

Thus, we have $\bar{s}=s\left(p^{e}\right)$ and thus assertions (b) and (c) follow.

We characterize the set of voucher prices in pooling equilibrium.

Theorem 5.3.2. Let $P^{e}$ be the set of prices that $R$ offers in pooling equilibrium, that is, $p^{e} \in P^{e}$ if there exists an a such that $\left(\left(p^{e}, p^{e}\right), a\right)$ is a pooling equilibrium. Then, it holds that

$$
P^{e}=\left\{p \in\left[t_{L}, t_{H}\right] \mid s(p) \geq s_{q}^{H}, F\left(s(p) \mid t_{L}\right)\left(t_{L}-p\right)+B \geq 0\right\}
$$

Proof. We first show the direction $\subseteq$.

If $P^{e}=\varnothing$, it trivially holds. Suppose $P^{e} \neq \varnothing$ and let $\hat{p} \in P^{e}$. The inequality $s(\hat{p}) \geq s_{q}^{H}$ is due to assertion (c) in Lemma 5.3.2. To see the other inequality, observe that the payoff of $t_{L}$-type in pooling equilibrium is $F\left(s(\hat{p}) \mid t_{L}\right)\left(t_{L}-\hat{p}\right)+B$. Since $R$ can always guarantee zero payoff by offering price equal 0 , it must be $F\left(s(\hat{p}) \mid t_{L}\right)\left(t_{L}-\hat{p}\right)+B \geq 0$. Therefore, we have

$$
\hat{p} \in\left\{p \in\left[t_{L}, t_{H}\right] \mid s(p) \geq s_{q}^{H}, F\left(s(p) \mid t_{L}\right)\left(t_{L}-p\right)+B \geq 0\right\} .
$$

We show the reverse direction $\supseteq$.

Choose $\hat{p} \in\left\{p \in\left[t_{L}, t_{H}\right] \mid s(p) \geq s_{q}^{H}, F\left(s(p) \mid t_{L}\right)\left(t_{L}-p\right)+B \geq 0\right\}$. Consider the strategy profile $((\hat{p}, \hat{p}), \hat{a})$ where

$$
\hat{a}(s, p)=\left\{\begin{array}{cl}
a^{s(\hat{p})}(s) & \text { if } p=\hat{p} \\
0 & \text { otherwise }
\end{array}\right.
$$

We claim that this strategy profile constitutes a pooling equilibrium.

1. We verify that offering $\hat{p}$ is a best response to $\hat{a}$. Assume that voucher holders use $\hat{a}$. When $R$ offers any price other than $\hat{p}$, no voucher holder will tender her voucher and so $R^{\prime}$ s payoff will be zero. On the 
other hand, when $R$ offers $\hat{p}$, voucher holders use the cutoff strategy at $s(\hat{p})$ and thus $t_{j}$-type $R$ will purchase the proportion of vouchers

$$
\alpha\left(\hat{a}, \hat{p}, t_{j}\right)=\mathbb{P}\left(s \leq s(\hat{p}) \mid t_{j}\right)=F\left(s(\hat{p}) \mid t_{j}\right) \quad(j=H, L) .
$$

This in turn implies that both types of $R$ succeed in gaining control because

$$
F\left(s(\hat{p}) \mid t_{L}\right) \geq F\left(s(\hat{p}) \mid t_{H}\right) \geq F\left(s_{q}^{H} \mid t_{H}\right)=q .
$$

where the first inequality holds since $F\left(. \mid t_{H}\right)$ first order stochastic dominates $F\left(. \mid t_{L}\right)$ and the second is by the condition $s(\hat{p}) \geq s_{q}^{H}$.

Hence, $t_{j}$-type $R^{\prime}$ s payoff is

$$
\begin{aligned}
u_{R}\left(\hat{p}, \alpha\left(\hat{a}, \hat{p}, t_{j}\right), t_{j}\right) & =u_{R}\left(\hat{p}, F\left(s(\hat{p}) \mid t_{j}\right), t_{j}\right) \\
& =F\left(s(\hat{p}) \mid t_{j}\right)\left(t_{j}-\hat{p}\right)+B \quad(j=H, L)
\end{aligned}
$$

Because $F\left(s(\hat{p}) \mid t_{H}\right)\left(t_{H}-\hat{p}\right)+B \geq F\left(s(\hat{p}) \mid t_{L}\right)\left(t_{L}-\hat{p}\right)+B \geq 0$, both types will have nonnegative payoffs. Thus, it is optimal for both types of $R$ to offer $\hat{p}$.

2. We next examine the optimality of $\hat{a}$ for voucher holder $i$. Assume that both types of $R$ offer $\hat{p}$ and all voucher holders other than $i$ use $\hat{a}$. Then,

$$
\left.\alpha\left(\hat{a}, \hat{p}, t_{H}\right)=F(s(\hat{p})) \mid t_{H}\right) \geq q
$$

and so the expected payoff of voucher holder $i$ when she tenders her voucher is $u\left(s_{i}\right)=\mathbb{P}\left(t_{H} \mid s_{i}\right) \cdot t_{H}+\mathbb{P}\left(t_{L} \mid s_{i}\right) \cdot t_{L}$. Hence, voucher holder $i$ will tender her voucher if and only if $\hat{p} \geq u\left(s_{i}\right)$ if and only if $s_{i} \geq s(\hat{p})$. As such, it is optimal for voucher holder $i$ to use the cutoff strategy at $s(\hat{p})$.

Summing up, $((\hat{p}, \hat{p}), \hat{a})$ is a pooling equilibrium and so $\hat{p} \in P^{e}$.

Therefore, the desired result (5.13) is established.

Using the theorem above, we verify that the set of equilibrium prices is a bounded and closed interval.

First, the inequality $s(p) \geq s_{q}^{H}$ in (5.13) gives the lower bound of the set $P^{e}$. Because the mapping $p \mapsto s(p)$ on $\left[t_{L}, t_{H}\right]$ is continuous and strictly 
increasing and its range is equal to $[0,1]$, there uniquely exists $p^{*} \in\left[t_{L}, t_{H}\right]$ such that

$$
s(p) \geq s_{q}^{H} \Longleftrightarrow p \geq p^{*}
$$

The intuition is that the price in equilibrium should be high enough to make voucher holders willing to tender their vouchers.

Second, the other inequality $F\left(s(p) \mid t_{L}\right)\left(t_{L}-p\right)+B \geq 0$ in (5.13) provides the upper bound of the set $P^{e}$. Notice that the mapping

$$
p \mapsto F\left(s(p) \mid t_{L}\right)\left(t_{L}-p\right)+B
$$

is continuous and strictly decreasing on $\left[t_{L}, t_{H}\right]$ and its range is $\left[t_{L}-t_{H}+\right.$ $B, B]$. Since it is assumed that $B \leq t_{H}-t_{L}$, there uniquely exists $p^{* *} \in\left[t_{L}, t_{H}\right]$ such that

$$
F\left(s(p) \mid t_{L}\right)\left(t_{L}-p\right)+B \geq 0 \Longleftrightarrow p \leq p^{* *}
$$

The upper limit of the price is required to prevent $t_{L}$-type $R$ from exiting the voucher market. It is because, if the voucher price is too high, $t_{L}$-type $R$ will give up buying vouchers and only $t_{H}$-type $R$ would remain in the market. Then due to the problem of free riding among voucher holders, there would be no equilibrium in the market.

Based on the observation above, we obtain the following corollary, which provides a necessary and sufficient condition for the existence of pooling equilibrium.

Corollary 5.3.1. A pooling equilibrium exists if and only if

$$
F\left(s\left(p^{*}\right) \mid t_{L}\right)\left(p^{*}-t_{L}\right) \leq B
$$

where $p^{*}$ is such that $s\left(p^{*}\right)=s_{q}^{H}$

Proof. According to Theorem 5.3.2, (5.15) and (5.16), $P^{e}=\left\{p \mid p^{*} \leq p \leq p^{* *}\right\}$. Hence, a pooing equilibrium exists if and only if $p^{*} \leq p^{* *}$. In turn, $p^{*} \leq p^{* *}$ if and only if $F\left(s\left(p^{*}\right) \mid t_{L}\right)\left(t_{L}-p^{*}\right)+B \geq 0$ by (5.16).

\subsection{Comparison of Voucher Privatization and Direct Privatization}

We have examined the equilibrium outcomes of voucher privatization. It is natural to look for an alternative mechanism which may yield a better 
outcome. We here consider direct privatization, the scheme that $G$ sells the firm directly to $R$. Our main interest is how much revenue $G$ can raise and which type of $R$ will gain control over the firm under this scheme.

\subsection{1 $G$ 's Maximum revenue in direct privatization}

The timeline of direct privatization is as follows.

1. Nature chooses $R^{\prime}$ s type $t$. The realized type is revealed only to $R$. $G$ has the belief that $R$ is of type $t_{H}$ with probability $\mu \in(0,1)$.

2. $G$ offers to $R$ a quantity-price menu $\left\{\left(\alpha_{H}, p_{H}\right),\left(\alpha_{L}, p_{L}\right)\right\}$, where $\alpha_{j} \in$ $[0,1]$ is the share fraction and $p_{j} \in \mathbb{R}$ the price per share for $j=H, L$.

3. $R$ decides whether to accept it. If $R$ accepts it, the contract is realized. Otherwise, $G$ keeps running the firm.

4. If $R$ purchases more than $q$ shares, $R$ gains control over the firm as a manager. Otherwise, $G$ keeps holding control over the firm.

When $G$ sells to $R$ the share $\alpha \in[0,1]$ at unit price $p$, the revenue of $G$ is

$$
U_{G}(\alpha, p, t)=\left\{\begin{array}{cl}
\alpha p+(1-\alpha) t & \text { if } \alpha \geq q \\
\alpha p+(1-\alpha) t_{G}+B & \text { otherwise }
\end{array}\right.
$$

where the first case is when $R$ manages the firm and the second when $G$ keeps managing the firm.

The payoff of $R$ is

$$
V_{R}(\alpha, p, t)=\left\{\begin{array}{cl}
\alpha t+B-\alpha p & \text { if } \alpha \geq q \\
\alpha t_{G}-\alpha p & \text { otherwise }
\end{array}\right.
$$

Our interest is to determine the optimal quantity-price menu for $G$. Let $\left\{\left(\alpha_{j}, p_{j}\right) \mid j=H, L\right\}$ be a quantity-price menu that $G$ offers to $R$. We say that the menu is incentive compatible (IC) if for $i, j \in\{H, L\}$

$$
V_{R}\left(\alpha_{i}, p_{i}, t_{i}\right) \geq V_{R}\left(\alpha_{j}, p_{j}, t_{i}\right)
$$

and individually rational (IR) if for $i \in\{H, L\}$

$$
V_{R}\left(\alpha_{i}, p_{i}, t_{i}\right) \geq 0 \text {. }
$$


The maximum (expected) revenue of $G$ is obtained by solving the following program:

$\max _{\left(\alpha_{H}, p_{H}\right),\left(\alpha_{L}, p_{L}\right)} \mu U_{G}\left(\alpha_{H}, p_{H}, t_{H}\right)+(1-\mu) U_{G}\left(\alpha_{L}, p_{L}, t_{L}\right)$ subject to (IC) and (IR).

Theorem 5.4.1. The quantity-price menu

$$
\left(\alpha_{H}, p_{H}\right)=\left(1, t_{H}+B\right) \text { and }\left(\alpha_{L}, p_{L}\right)=(0,0)
$$

is optimal. Under this menu, the payoffs of $G$ and $R$ are $\mu t_{H}+(1-\mu) t_{G}+B$ and 0 , respectively.

Proof. Observe that the maximum of $G^{\prime}$ s payoff cannot be above $\mu t_{H}+(1-$ $\mu) t_{G}+B$. It is because

$$
U_{G}(\alpha, p, t)+V_{R}(\alpha, p, t)=\left\{\begin{array}{cl}
t+B & \text { if } \alpha \geq q \\
t_{G}+B & \text { otherwise }
\end{array}\right.
$$

and thus

$$
\begin{aligned}
\mu U_{G}\left(\alpha_{H}, p_{H}, t_{H}\right)+(1-\mu) U_{G}\left(\alpha_{L}, p_{L}, t_{L}\right)= & \mu\left(t_{H}+B\right)+(1-\mu)\left(t_{G}+B\right) \\
& -\mu V_{R}\left(\alpha_{H}, p_{H}, t_{H}\right)-(1-\mu) V_{R}\left(\alpha_{L}, p_{L}, t_{L}\right) \\
\leq & \mu\left(t_{H}+B\right)+(1-\mu)\left(t_{G}+B\right)
\end{aligned}
$$

where the inequality is due to (IC).

We show the upper bound $\mu t_{H}+(1-\mu) t_{G}+B$ can be achieved by the menu (5.21). Indeed, under (5.21),

$V_{R}\left(\alpha_{H}, p_{H}, t_{H}\right)=0, V_{R}\left(\alpha_{L}, p_{L}, t_{L}\right)=0, V_{R}\left(\alpha_{L}, p_{L}, t_{H}\right)=0, V_{R}\left(\alpha_{H}, p_{H}, t_{L}\right)=t_{L}-t_{H}$

and thus (IC) and (IR) are satisfied. The desired result follows.

The point of the theorem above is that $G$ can perfectly distinguish $R^{\prime}$ s types and thus choose not to sell the firm to $t_{L}$-type $R$. 


\subsubsection{Comparison}

We compare the outcomes of direct privatization and voucher privatization and compare them from an economic as well as a political perspective. Throughout this section, we assume that (5.17) holds and so a pooling equilibrium exits in voucher privatization. Recall that an outcome is efficient if and only if $t_{H}$-type $R$ gains control over the firm and $t_{L}$-type does not.

Theorem 5.4.2. The outcome of direct privatization is always efficient. The equilibrium outcome of voucher privatization can be inefficient.

Proof. In direct privatization, $G$ sells the firm only to $t_{H}$-type $R$ by Theorem 5.4.1. Hence, the outcome is efficient.

In voucher privatization, by the third claim of Theorem 5.3.1 one of the outcomes in separating equilibrium is that no voucher trade occurs and so neither type fails to run the firm. In pooling equilibrium, by Lemma 5.3.2 both types of $R$ gain control over the firm. Hence, the equilibrium outcome under voucher privatization can be inefficient.

This finding argues that voucher privatization is not a good choice from the economic efficiency point of view. Of course, the loss of economic efficiency can be excused to some degree because the reason that voucher privatization was chosen over the standard economic mechanisms was that the government aimed to gain public support rather than to achieve economic efficiency. Then, the natural question is whether, at the loss of efficiency, voucher privatization can serve the political goal better than direct privatization with redistributing to the public the proceeds of selling the firm. To answer this question, we compare the sum of total payoffs to citizens and $G$ under voucher privatization with $G$ 's revenue under direct privatization.

Theorem 5.4.3. The sum of payoffs of voucher holders and $G$ under direct privatization is strictly higher than the sum of equilibrium payoffs to them under voucher privatization.

Proof. Under direct privatization, voucher holders have zero payoff and, as seen in Theorem 5.4.1, $G$ gets the payoff of $\mu t_{H}+(1-\mu) t_{G}+B$. Thus, the 
sum of two is simply

$$
\mu t_{H}+(1-\mu) t_{G}+B
$$

We compare (5.22) with the sum of equilibrium payoffs to them $G$ under voucher privatization.

(Separating equilibrium) By the first and second claims of Theorem 5.3.1, the sum of payoffs to voucher holders and $G$ is either $t_{G}+B$ or $\mu t_{H}+$ $(1-\mu)\left(t_{G}+B\right)$. Both are strictly lower than (5.22).

(Pooling equilibrium) Let $p^{e}$ be any price in pooling equilibrium. The total sum of all voucher holders' payoffs in voucher privatization conditional on $t=t_{j}(j=H, L)$ is equal to

$$
\begin{aligned}
p^{e} \int_{\left\{i: a\left(s_{i}, p^{e}\right)=1\right\}} d i+t_{j} \int_{\left\{i: a\left(s_{i}, p^{e}\right)=0\right\}} & =p^{e} \int_{\left\{s: s \leq s\left(p^{e}\right)\right\}} d i+t_{j} \int_{\left\{s: s \leq s\left(p^{e}\right)\right\}} \quad(\text { by Lemma 5.3.2) } \\
& =F\left(s\left(p^{e}\right) \mid t_{j}\right) p^{e}+\left(1-F\left(s\left(p^{e}\right) \mid t_{j}\right)\right) t_{j} \\
& =F\left(s\left(p^{e}\right) \mid t_{j}\right)\left(p^{e}-t_{j}\right)+t_{j}
\end{aligned}
$$

where the first (second, resp.) integral is taken over payoffs to voucher holders who tender (keep, resp.) their vouchers.

As for $G$, since in pooling equilibrium the firm will be run by $R, G$ gets zero payoff.

Thus, the sum of expected payoffs to them is equal to

$$
\mu\left[F\left(s\left(p^{e}\right) \mid t_{H}\right)\left(p^{e}-t_{H}\right)+t_{H}\right]+(1-\mu)\left[F\left(s\left(p^{e}\right) \mid t_{L}\right)\left(p^{e}-t_{L}\right)+t_{L}\right] .
$$

This is strictly lower than (5.22) because

$$
\begin{aligned}
\left(\mu t_{H}+(1-\mu) t_{G}+B\right) & -\left(\mu\left[F\left(s\left(p^{e}\right) \mid t_{H}\right)\left(p^{e}-t_{H}\right)+t_{H}\right]+(1-\mu)\left[F\left(s\left(p^{e}\right) \mid t_{L}\right)\left(p^{e}-t_{L}\right)+t_{L}\right]\right) \\
& =\mu\left[B+F\left(s\left(p^{e}\right) \mid t_{H}\right)\left(t_{H}-p^{e}\right)\right]+(1-\mu)\left[B+F\left(s\left(p^{e}\right) \mid t_{L}\right)\left(t_{L}-p^{e}\right)\right] \\
& +(1-\mu)\left(t_{G}-t_{L}\right) \\
& >0 .
\end{aligned}
$$

To see the inequality, observe that $B+F\left(s\left(p^{e}\right) \mid t_{H}\right)\left(t_{H}-p^{e}\right)>0$ because $p^{e} \leq$ $t_{H}$ and $B+F\left(s\left(p^{e}\right) \mid t_{L}\right)\left(t_{L}-p^{e}\right) \geq 0$ by Theorem 5.3.2. In addition, $t_{G} \geq t_{L}$.

Putting all together, we obtain the desired result. 
The theorem above implies that in the terms of citizens' payoff, it is a better privatization scheme for the government to sell the firm directly and distribute the revenue from the trade to citizens than voucher privatization. This suggests that if the political support of the public is proportional to their payoff, the government could have gained stronger support by transferring to them the proceeds of direct privatization.

\subsection{Conclusion}

The main reason voucher privatization was chosen in the 1990s over the standard auctions was political rather than economic. We then face two questions. Has economic efficiency fallen victim to political feasibility? Did voucher privatization raise the maximum revenue?

This chapter tried to answer these questions from the perspective of signaling games. We verified that there does not exist a separating equilibrium and a raider who is less able than a government may succeed in acquiring the state-owned firm. We also showed that bilateral trade between a government and a raider would give the government more revenue than voucher privatization scheme. 


\section{Bibliography}

Armstrong, M. (1996). Multiproduct nonlinear pricing. Econometrica 64(1), 51-75.

Bolton, P. and M. Dewatripont (2005). Contract Theory. Cambridge: The MIT Press.

Boycko, M., A. Shleifer, and R. W. Vishny (1993). Privatizing russia. Brookings Papers on Economic Activity 24(2), 139-192.

Boycko, M., A. Shleifer, and R. W. Vishny (1994). Voucher privatization. Journal of Financial Economics 35(2), 249-266.

Cai, H., J. G. Riley, and L. Ye (2007). Reserve price signaling. Journal of Economic Theory 135(1), 253-268.

Carbajal, J. C. and J. C. Ely (2013). Mechanism design without revenue equivalence. Journal of Economic Theory 148(1), 104-133.

Chowdhry, B. and N. Jegadeesh (1994). Pre-tender offer share acquisition strategy in takeovers. Journal of Financial and Quantitative Analysis 29(01), 117-129.

Constantinides, G. M. and B. Grundy (1989). Optimal investment with stock repurchase and financing as signals. Review of Financial Studies 2(4), 445-465. 
DeMarzo, P. and D. Duffie (1999). A liquidity-based model of security design. Econometrica 67(1), 65-100.

Dubey, P., J. Geanakoplos, and M. Shubik (2005). Default and punishment in general equilibrium. Econometrica 73(1), 1-37.

Engers, M. (1987). Signalling with many signals. Econometrica 55(3), 66374.

Fehr, E. and K. M. Schmidt (1999). A theory of fairness, competition, and cooperation. Quarterly Journal of Economics 114(3), 817-868.

Grossman, S. J. and O. D. Hart (1980). Takeover bids, the free-rider problem, and the theory of the corporation. Bell Journal of Economics 11(1), 42-64.

Gul, F. (1991). A theory of disappointment aversion. Econometrica 59(3), $667-86$.

Hartman, P. (1982). Ordinary Differential Equations. Berlin: Birkhäuser.

Heydenreich, B., R. Müller, M. Uetz, and R. V. Vohra (2009). Characterization of revenue equivalence. Econometrica 77(1), 307-316.

Hirshleifer, D. and S. Titman (1990). Share tendering strategies and the success of hostile takeover bids. Journal of Political Economy 98(2), 295324.

Hoppe, H. C., B. Moldovanu, and A. Sela (2009). The theory of assortative matching based on costly signals. Review of Economic Studies 76(1), 253281.

Johnstone, R. A. (1995). Honest advertisement of multiple qualities using multiple signals. Journal of Theoretical Biology 177(1), 87-94.

Jullien, B. and T. Mariotti (2006). Auction and the informed seller problem. Games and Economic Behavior 56(2), 225-258.

Katz, B. G. and J. Owen (1997). Optimal voucher privatization fund bids when bidding affects firm performance. Journal of Comparative Economics 24(1), 25-43. 
Kim, J.-Y. (2007). Multidimensional signaling in the labor market. Manchester School 75(1), 64-87.

Köszegi, B. and M. Rabin (2006). A model of reference-dependent preferences. Quarterly Journal of Economics 121(4), 1133-1165.

Kreps, D. M. and J. Sobel (1994). Signalling. In R. Aumann and S. Hart (Eds.), Handbook of Game Theory with Economic Applications, Chapter 25, pp. 849-867. Elsevier.

Krishna, V. (2002). Auction Theory. New York: Academic Press.

Mailath, G. J. (1987). Incentive compatibility in signaling games with a continuum of types. Econometrica 55(6), 1349-65.

Mailath, G. J. and E.-L. von Thadden (2013). Incentive compatibility and differentiability: New results and classic applications. PIER Working Paper Archive 10-032, Penn Institute for Economic Research, University of Pennsylvania.

Marquez, R. and B. Yilmaz (2008). Information and efficiency in tender offers. Econometrica 76(5), 1075-1101.

Milgrom, P. and J. Roberts (1986). Price and advertising signals of product quality. Journal of Political Economy 94(4), 796-821.

Mishra, D. and D. Talman (2010). Characterization of the walrasian equilibria of the assignment model. Journal of Mathematical Economics 46(1), $6-20$.

Prescott, E. C. and R. M. Townsend (1984). Pareto optima and competitive equilibria with adverse selection and moral hazard. Econometrica 52(1), 21-46.

Quinzii, M. and J.-C. Rochet (1985). Multidimensional signalling. Journal of Mathematical Economics 14(3), 261-284.

Riley, J. G. (1979). Informational equilibrium. Econometrica 47(2), 331-59.

Riley, J. G. (2001). Silver signals: Twenty-five years of screening and signaling. Journal of Economic Literature 39(2), 432-478. 
Rudin, W. (1976). Principles of mathematical analysis (Third ed.). New York: McGraw-Hill.

Salanié, B. (2005). The Economics of Contracts: A Primer, 2nd Edition. Cambridge: The MIT Press.

Shleifer, A., R. W. Vishny, A. Shleifer, and R. W. Vishny (1986). Large shareholders and corporate control. Journal of Political Economy 94, 461-488.

Sobel, J. (2009). Signaling games. In Encyclopedia of Complexity and Systems Science. Springer.

Spence, M. (1973). Job market signaling. Quarterly Journal of Economics 87(3), 355-74.

Vohra, R. (2011). Mechanism Design: A Linear Programming Approach. Econometric Society Monographs. Cambridge University Press.

Wilson, R. (1985). Multi-dimensional signalling. Economics Letters 19(1), $17-21$.

Yusupova, E. (2006). Information asymmetry, share mispricing and the coordination problem: Investor portfolio choice in czech voucher privatization. CERGE-EI Working Papers 301, The Center for Economic Research and Graduate Education - Economic Institute. 


\section{Nederlandse samenvatting}

In het eerste gedeelte van dit proefschrift (hoofdstukken 2, 3, en 4) geven we een karakterisatie van een zogeheten separating evenwicht voor standaard signaling games, waar de uitbetaling van de zender lineair afhangt van de actie die wordt gekozen door de ontvanger. In hoofdstukken 2 en 3 beschouwen we signaling games waar de dimensie van zowel de ruimte van typen als die van de signalen gelijk is aan 1 . Gegeven een strategie van de zender construeren we een netwerk waar de verzameling knooppunten gelijk is aan de verzameling typen van de zender, en de lengte van een kant gegeven wordt door het verschil in kosten tussen eindpunt en beginpunt van de kant in kwestie. Dit laat zien dat het vinden van een separating evenwicht equivalent is aan het construeren van een lengtefunctie in het netwerk, onder de voorwaarde dat de strategie van de ontvanger een potentiaal is voor deze lengtefunctie.

We tonen aan dat, indien de verzameling typen van de zender een reÃnel interval is, de lengte van het kortste pad in het netwerk antisymmetrisch is, en dat de potentiaalfunctie uniek is modulo een contante. De evenwichtsstrategie van de zender in een separating evenwicht wordt dan gegeven door een differentiaalvergelijking die zoals we laten zien een unieke oplossing heeft. We leiden ook voldoende voorwaarden af voor het bestaan en de uniciteit van een separating evenwicht.

Onze resultaten die, afgezien van de eerder genoemde lineariteit, zwakkere condities gebruiken dan Mailath (1987) en Riley (1979), kunnen worden toegepast op een breed scala van economische problemen. In hoofdstuk 
2 behandelen we het geval waar de uitbetaling van de ontvanger niet afhangt van de keuze van het signaal. In hoofdstuk 3 bekijken we de meer algemene situatie waar de keuze (kwaliteit) van het signaal invloed heeft op de uitbetaling van de ontvanger.

In hoofdstuk 4 beschouwen we signaling games waar zowel de typen van de zender als het signaal meerdimensionaal kunnen zijn. We geven, voor een grote klasse van kostenfuncties, een exacte karakterisatie voor het bestaan van een separating evenwicht. We tonen in het bijzonder aan dat de strategie van de zender in een separating evenwicht berekend kan worden als de oplossing van een bepaald minimalisatieprobleem, vergelijkbaar met de afleiding van Hicks vraagfuncties. Ons model kan gezien worden als een generalisatie van Quinzii en Rochet (1985), die een karakterisatie geven van separating evenwicht in het geval van scheidbare lineaire kostenfuncties. We leiden sommige van hun resultaten af uit onze karakterisatie.

In hoofdstuk 5 gebruiken we signaling games om voucher privatisatie te analyseren. Begin 1990 werd in Rusland een voucher veiling gebruikt om staatsbedrijven te privatizeren. Ondanks veelvuldig gebruik van dit mechanisme heeft het tot nu toe relatief weinig aandacht gekregen in de literatuur. We beschouwen een model waar een bedrijf kan worden geprivatiseerd. In deze context karakteriseren we de verzameling voucher evenwichtsprijzen. We vergelijken voucher privatisatie met directe privatisatie, en concluderen dat voucher privatisatie in dit geval inferieur is, zowel in economisch als in politiek opzicht. 


\section{Short Curriculum Vitae}

Jiwoong Lee was born in March, 1977 in Damyang, South Korea. After attending Gwangju high school of science, he entered Seoul National University, in which he earned a bachelor degree in mathematics. He worked as a junior economist for the Bank of Korea (Korean central bank) in Seoul and performed military duties as a naval officer for three years. After being discharged, he moved to France and attained a research master degree in mathematical economics and econometrics with distinction at Toulouse School of Economics.

Thereafter, he started his doctoral studies at Maastricht University, the Netherlands in January 2009.

Jiwoong's research focuses on game theory and mechanism design. 